This is a preprint version from January 2015 of a review article that was published in August 2015. According to the authors' copyright agreement with the publisher, the final published version CANNOT

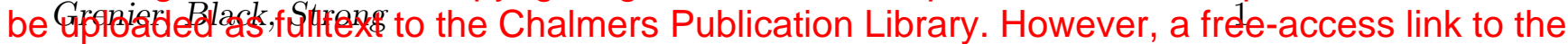
publisher's fulltext is included in the "link to fulltext" in the CPL entry. Posted with permission from the Annual Review of Astronomy and Astrophysics, Volume 53 ( 2015 by Annual Reviews, http:// www.annualreviews.org.

\title{
The Nine Lives of Cosmic Rays in Galaxies
}

\author{
Isabelle A. Grenier ${ }^{1}$, John H. Black ${ }^{2}$, Andrew W. Strong ${ }^{3}$, \\ 1. Laboratoire AIM Paris-Saclay, CEA/Irfu, CNRS, Université Paris Diderot, \\ F-91191 Gif-sur-Yvette Cedex, France. isabelle.grenier@cea.fr \\ 2. Department of Earth \& Space Sciences, Chalmers University of Technology, \\ Onsala Space Observatory, SE-439 92 Onsala, Sweden. john.black@chalmers.se \\ 3. Max-Planck-Institut für extraterrestrische Physik, Postfach 1312, 85741 \\ Garching, Germany. aws@mpe.mpg.de
}

Key Words cosmic rays, $\gamma$ rays, interstellar medium, interstellar chemistry, dust, superbubbles

\begin{abstract}
Cosmic-ray astrophysics has advanced rapidly in recent years, and its impact on other astronomical disciplines has broadened. Many new experiments, measuring the particles both directly in the atmosphere or space, and indirectly via $\gamma$ rays and synchrotron radiation, have widened the range and quality of the information available on their origin, propagation, and interactions. The impact of low-energy cosmic rays on interstellar chemistry is a fast-developing topic, including the propagation of these particles into the clouds where the chemistry occurs. Cosmic rays, via their $\gamma$-ray production, also provide a powerful way to probe the gas content of the interstellar medium. Substantial advances have been made in the observations and modelling of the interplay between cosmic rays and the interstellar medium. Focussing on energies up to $1 \mathrm{TeV}$, these inter-relating aspects are covered at various levels of detail, giving a guide to the state of the subject.
\end{abstract}

\section{CONTENTS}

Scope of the review . . . . . . . . . . . . . . . . . . . 2

Advances from direct measurements . . . . . . . . . . . . . . . . . . . . 4

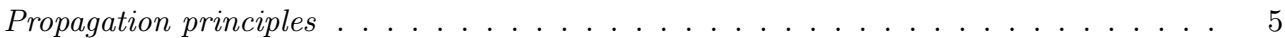

Composition of primary cosmic rays . . . . . . . . . . . . . . . . 6

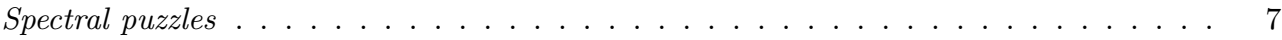

Positron excess at high energies . . . . . . . . . . . . . . . . . 8

Advances in cosmic-ray propagation . . . . . . . . . . . . . . . . . . 9

Advances in propagation physics . . . . . . . . . . . . . . . . . . . . 11

Advances in propagation models . . . . . . . . . . . . . . . . . . . . . . . . 12

Cosmic-ray wanderers in the Milky Way . . . . . . . . . . . . . . . . . 13

Cosmic rays in the local ISM . . . . . . . . . . . . . . . . . . . . . . . . . . . . . . . . . . . . . . . . . . . . . . . . .

Cosmic rays in the outer Galaxy . . . . . . . . . . . . . . . . . . . . . . . 16

Cosmic rays in the inner Galaxy . . . . . . . . . . . . . . . . . . . . . . . . 17

Global properties of the high-energy Galaxy . . . . . . . . . . . . . . . . . . 19

Cosmic-ray/ISM interaction processes . . . . . . . . . . . . . . . . 19 
Atomic and molecular processes . . . . . . . . . . . . . . . . . . . . . . 19

Cosmic-ray-induced ultraviolet radiation . . . . . . . . . . . . . . . . . . . 21

Interaction with solid particles . . . . . . . . . . . . . . . . . . . 22

Spallation and synthesis of light elements . . . . . . . . . . . . . . 22

Cosmic-ray Stimuli on the ISM . . . . . . . . . . . . . . . . . . . . . . . . . . . . . . . . . . 23

In the vicinity of cosmic-ray sources . . . . . . . . . . . . . . . . . . . . . . 23

Interstellar ion chemistry driven by cosmic rays . . . . . . . . . . . . . . . . 25

Observations of molecular ions: $H_{3}^{+} \ldots \ldots \ldots \ldots \ldots \ldots$

Observations of molecular ions: $\mathrm{OH}^{+}, \mathrm{H}_{2} \mathrm{O}^{+}, \mathrm{H}_{3} \mathrm{O}^{+} \ldots \ldots \ldots \ldots$

Cosmic-ray tracers of the ISM . . . . . . . . . . . . . . . . . . . . . . 28

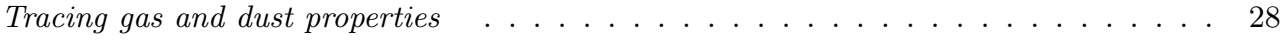

Tracing cosmic-ray leptons and the magnetic field . . . . . . . . . . . . . . . . . 31

Cosmic rays in star-burst environments . . . . . . . . . . . . . . . . . . 32

Effect on cosmic-ray composition . . . . . . . . . . . . . . . . . . . . . . 32

A cocoon of young cosmic rays in Cygnus X . . . . . . . . . . . . . . . . . 33

Normal galaxies . . . . . . . . . . . . . . . . . . . . . . 34

Starburst galaxies . . . . . . . . . . . . . . . . . . . . . . 34

$\gamma$-rays in extragalactic space . . . . . . . . . . . . . . . 35

A few more words . . . . . . . . . . . . . . . . . . 36

Related Resources ......................... 36

Acknowledgements . . . . . . . . . . . . . . . . . . . 36

\section{Scope of the review}

The term cosmic rays (CRs) refers generically to particles with energies starting at $\mathrm{MeV}$ and continuing to around $10^{21} \mathrm{eV}$. The particles encompass nuclei (from protons to actinides), antiprotons, electrons, and positrons. Locally, the CR energy density is dominated by $\mathrm{GeV}$ protons and compares with the interstellar energy densities of the thermal gas, magnetic field, stellar radiation, and the cosmic microwave background. The Larmor radii of Galactic CRs range from $10^{5} \mathrm{~km}$ at the lowest energies to $10^{-1}$ parsec near $10^{15} \mathrm{eV}$, so that directional information on their sources is completely lost during propagation. For this reason, $\gamma$-ray and radio synchrotron observations are essential to study the large-scale distribution and spectra of Galactic CRs, giving clues to their origin and propagation. There is good evidence that CRs are accelerated in Galactic sites up to the knee energy of $\sim 3 \times 10^{15} \mathrm{eV}$ where the spectrum steepens; at much higher energies they almost certainly originate outside the Galaxy since the Larmor radii are of order $\mathrm{kpc}$ above $10^{18} \mathrm{eV}$ and there is no sign of a directional modulation related to the Galactic disc. The transition energy from Galactic to extragalactic, however, is not known because the nuclear compositions of both Galactic and extragalactic CRs affect the spectrum in the transition region through the dependence of the Larmor radius on mass and charge. The transition is usually assumed to be in the $10^{15-17} \mathrm{eV}$ band where the CR spectrum steepens from index 2.7 to around 3.3 (in particle flux per momentum unit), consistent with the point of rapid escape from the Galaxy. The spectrum flattens again at higher energies, consistent with a contribution from extragalactic sources. CR electrons and positrons, on the other hand, are all certainly of Galactic origin since their energy losses by inverse 
Compton scattering on the Cosmic Microwave Background limit their travel in intergalactic space to only a few $\mathrm{kpc}$, even at $\mathrm{GeV}$ energies.

The centenary of the 1912 discovery of cosmic rays has passed, accompanied by commemorative celebrations (Ormes 2013) and reviews (Blandford, Simeon \& Yuan 2014; Blasi 2013; Kotera \& Olinto 2011; Slane et al. 2014; Zweibel 2013), which highlighted the impressive historical progress on the continuing questions of their origin and acceleration, in the Galaxy and beyond. The main acceleration site of Galactic CRs is thought to be the shocks of young supernova remnants (SNR). Particles traverse the shock many times due to efficient, Bohm-like, diffusion. They gain momentum at each pass, generating a power-law spectrum. The number of remnants and the long-term average supernova frequency in the Galaxy are sufficient to supply the observed CRs if a few per cent of the explosive energy of a supernova is converted into CRs (e.g., Drury, Aharonian \& Voelk 1994). Gamma-ray observations of supernova remnants at $\mathrm{GeV}$ energies (Fermi-LAT, AGILE) and at TeV energies (H.E.S.S., MAGIC, VERITAS) allow direct tests of this scenario via the production of pion-decay, bremsstrahlung, and inverse-Compton $\gamma$ rays by in-situ CRs. The challenge is to separate and delineate the emission components from the CR nuclei and electrons, i.e. to probe how efficiently and how high in energy supernova shock waves can accelerate both types of particles. A hadronic emission signature has at last been found in the W44 and IC443 remnants with Fermi-LAT and AGILE (Ackermann et al. 2013b, Cardillo et al. 2014), although it will not constitute firm evidence of the acceleration of CR nuclei until the measurements are extended to well below the pion peak energy at $70 \mathrm{MeV}$.

In parallel, a significant paradigm shift has been the realization that CR sources other than SNRs are probably important. Pulsars and pulsar-wind nebulae are now receiving more attention as sources of primary electrons and positrons, triggered by observations of more high-energy positrons than expected from hadronic interactions of CRs with interstellar matter (see Section 2.4).

CR sources and acceleration mechanisms are not addressed in the present review. For recent coverage, we refer the reader to the insightful reviews quoted above, and to Castellina \& Donato (2013) and Amato (2014).

We focus instead on the numerous facets of cosmic-ray lives and feedback in their host galaxies, which we attempt to capture and review here, in their fascinating variety. In broad brushstrokes, Galactic cosmic rays ...

1. differentially penetrate into astrospheres to probe their physics, as glimpsed in the heliosphere by the two Voyager spacecraft and by $\gamma$-ray observations toward the Sun.

2. randomly diffuse through the turbulent interstellar magnetic fields, scrambling their paths to our detectors, but transferring and ultimately depositing energy across kiloparsecs in the interstellar medium (ISM). CR nuclei and electrons respectively leave $10 \%$ and $60 \%$ of their energy before escaping into intergalactic space. Energy losses and interactions with matter and radiation fields leave collective traces of their long journey through the Galactic disc and halo.

3. produce spallation secondaries with elemental and isotopic abundances of diagnostic value in understanding the composition of accelerated matter and later propagation.

4. drive MHD waves, which partly maintain interstellar turbulence. Con- 
versely, CRs are subjected to wind-driven turbulence near stellar clusters, which may severely reduce their diffusion lengths in star-forming regions.

5. drive large-scale interstellar flows, including possible galactic winds, fountains, and perhaps large-scale features like the 'Fermi Bubbles'.

6. take away between $10 \%$ and $50 \%$ of the energy of the supernova shockwaves that have accelerated them, thereby lowering the remnant temperatures and moderating their feedback on the ISM.

7. ionise and heat the dense interstellar gas, depositing $\sim 13 \mathrm{eV}$ per interaction to keep the darkest ISM at temperatures near $10 \mathrm{~K}$ and electron fractions near $10^{-7}$. They drive a remarkably rich chemistry at low temperature. Reactive molecular ions thus provide remote tracers of low-energy CRs, with kinetic energies below a few $\mathrm{MeV}$.

8. cause interstellar clouds to glow brightly in $\gamma$ rays and reveal their total gas content, irrespective of their chemical and thermodynamical state.

9. interact with magnetic fields, probing their strength, and with soft radiation fields to trace starlight and dust radiation.

We have highlighted nine topics above, hence the title of this review ${ }^{1}$ which aims to discuss recent findings on the numerous interactions of sub-TeV CRs with their interstellar habitat.

Many of these findings were not known at the time of the previous CR article in Annual Reviews (Strong, Moskalenko \& Ptuskin 2007), which illustrates how lively this field of research is. New results include direct composition measurements from light to heavy nuclei; evidence of spectral hardening above a few hundred $\mathrm{GeV}$ and of spectral differences between the nuclei; the successful mapping of remote CRs in the Milky Way from their $\gamma$-ray emission, as seen by the Fermi LAT telescope; the rapid developments in the use of chemical information to probe few-MeV CRs beyond the enormous barrier of the heliosphere and near supernova remnants; rapid progress in the use of CRs and their $\gamma$-ray emission to trace the total gas in the ISM; the recent realization of the potential confinement of young CRs in starburst regions; and the first observations of CR-driven $\gamma$ rays in starburst galaxies. The review focusses on CR energies only up to $1 \mathrm{TeV}$, since it is mainly concerned with the interstellar effects of CRs and their use as a tracer of the ISM, where only lower energies are relevant.

\section{Advances from direct measurements}

In CR astrophysics the most important resources are the experiments and their data. Direct measurements are those made from balloons in the atmosphere and spacecraft in the heliosphere, in contrast to indirect methods using $\gamma$ rays, synchrotron, etc. to remotely probe CRs to large distances in the Galaxy. Improved experimental techniques have led to significant progress particularly in the quality and accuracy of the measured composition and spectra. In some cases there is no longer any significant experimental uncertainty remaining for the theorist using these data; therefore, the challenges for interpretation are well defined.

Table 1 summarizes recent direct measurements discussed below and gives their references.

\footnotetext{
${ }^{1}$ for completeness we mention that there are other related aspects like the effect of $\mathrm{CR}$ on climate and weather, not addressed here.
} 
Direct CR observations from balloons and satellites are affected by the solar wind below a few $\mathrm{GeV}$, which makes their astrophysical interpretation problematic, but at the same time allows detailed studies of solar modulation. Solar modulation is an important topic not covered in this review. A recent study using time-dependent measurements of proton and Helium spectra with PAMELA, and a comparison with heliospheric models, can be found in Adriani et al. (2013c), to which the reader is referred for further literature.

Another important finding is the observation of anisotropies in the CR flux at $\mathrm{TeV}$ energies and above. It relates to diffusion from discrete CR sources, but also to the structure of the local interstellar magnetic field and of the heliosphere, and it is not included in the present review (see e.g. Pohl \& Eichler 2013, Schwadron et al. 2014, for further reading).

Until a few years ago surprisingly little attention was devoted to the task of collecting CR data in a systematic way, given the huge effort expended in building and deploying instruments to make the measurements. An initiative started in 2009 (Strong \& Moskalenko 2009) has been pursued for several years and is now superseded by a sophisticated database, which is maintained with incoming data and offers comprehensive literature and instant plots (Maurin, Melot \& Taillet 2014). Interested readers are recommended to access this valuable resource ${ }^{2}$.

Our understanding of CR data in terms of physical models is still rather rudimentary. For orientation we give a general introduction to the principles involved before commenting recent results.

\subsection{Propagation principles}

Galactic CRs are thought to propagate mainly by diffusion due to scattering on random or turbulent magnetic fields. The spatial diffusion coefficient, $D_{x x}(p)$, depends on particle momentum $p$. Regular transport by convection in Galactic winds and fountains is also probable. The particle spectra are modified along the way by energy losses and by diffusive reacceleration. The latter is due to particle scattering on moving MHD waves. Random wave motions lead to stochastic acceleration, which is described as diffusion in momentum space with diffusion coefficient $D_{p p}$. Energy losses include a large variety of processes, such as ionization and Coulomb interactions with interstellar gas (dominant for low-energy CR nuclei) and hadronic interactions like pion production (dominant for high-energy nuclei). Lepton losses are much larger due to synchrotron, bremsstrahlung, and inverse-Compton interactions, as well as ionization and Coulomb losses. The CR nuclei produced by the sources (primary CR) also undergo nuclear interactions with the interstellar gas, producing secondary nuclei by spallation (secondary CR). CR dynamics in the Galaxy is therefore generally described by a differential equation which includes acceleration, loss, and transport terms (see e.g. Strong, Moskalenko \& Ptuskin 2007; Zweibel 2013).

CR sources are concentrated in the Galactic plane, and the particles undergo great numbers of scatterings before finally escaping into intergalactic space. The escape is an essential, but little understood, part of their history, and is usually treated simply by setting the CR density to zero at some boundary, a few kpc above the Galactic plane. Its height can be tested in Galactic-wide propagation models. The region occupied by CRs is often referred to as the CR halo, and

\footnotetext{
2at http://lpsc.in2p3.fr/cosmic-rays-db
} 
similar halos are observed in the radio synchrotron emission from external galaxies viewed edge-on (Beck 2012).

\subsection{Composition of primary cosmic rays}

The primary $\mathrm{CR}$ composition can be determined from direct measurements, after accounting for secondary spallation products during Galactic propagation. The latest elemental composition data are from ACE, CREAM, TRACER, and TIGER (see Table 1 for details and references).

These data concur to suggest that CRs are accelerated from material of the general interstellar medium, mixed with only a small fraction of stellar wind material expelled by the massive progenitors of core-collapse supernovae. The evidence for this is the generally similar composition of CRs to that of the solar photosphere or meteorites, after allowing for spallation secondaries.

Deviations from interstellar composition are nevertheless observed, and are related to primary CR acceleration via first-ionization potential, volatility, and the isotopic composition in special regions like superbubbles. Elemental and isotopic abundances therefore give essential clues to the acceleration sites and time scales. The recent data confirm the CR enrichment, relative to solar abundances, in refractory elements over volatile ones. They also confirm that elements with first ionization potentials larger than $10 \mathrm{eV}$ are depleted with respect to those with lower potentials, up to TeV energies per nucleon (Ahn et al. 2010, Rauch et al. 2009).

Yet, the observed dependence of abundances on atomic mass or charge is not fully explained by ionization potentials and volatility, and some elemental abundances (e.g. $\mathrm{Ga} / \mathrm{Ge}$ ) are inconsistent with a pure interstellar composition. The organization of the data significantly improves when using a CR composition mixture of about $20 \%$ ejecta of massive stars (including Wolf-Rayet winds and core-collapse supernovae) and 80\% interstellar gas (Rauch et al. 2009). If so, the acceleration mechanism should be 3 to 4 times more efficient for refractory elements than for volatiles. This is possible via the acceleration of interstellar grains, as suggested by Ellison, Drury \& Meyer (1997). In this model, dust grains are charged by photo-ionization and accelerated to modest energies by supernova shock waves. Atoms later sputtered off of these grains are injected in the accelerating shock with supra-thermal energies, hence they are more efficiently accelerated than thermal interstellar atoms swept up and heated by the shock. The grain model has unfortunately not been pursued lately.

The large isotopic ratio of ${ }^{22} \mathrm{Ne} /{ }^{20} \mathrm{Ne}$, which is $5.3 \pm 0.3$ times higher in CRs than in the solar system, also provides useful constraints on CR acceleration, suggesting that acceleration be restricted in time to the early periods of the SNR expansion, ending well before the end of the Sedov phase (Prantzos 2012). The high ${ }^{22} \mathrm{Ne} /{ }^{20} \mathrm{Ne}$ ratio together with the lack of the unstable ${ }^{59} \mathrm{Ni}$ in CRs (Wiedenbeck et al. 1999) yield important time constraints for CR acceleration in massive-star clusters: it should take place soon $(<$ few Myr) after enrichment of the ambient medium in ${ }^{22} \mathrm{Ne}$ by the Wolf-Rayet winds, and about $0.1 \mathrm{Myr}$ after a supernova explosion to avoid accelerating freshly synthesized ${ }^{59} \mathrm{Ni}$. This may be possible if the injections of ${ }^{22} \mathrm{Ne}$-rich wind material and of ${ }^{59} \mathrm{Ni}$-rich supernova ejecta are sufficiently separated in time (Binns et al. 2007) and if ${ }^{59} \mathrm{Ni}$ largely remains inside the supernova ejecta, beyond reach of the forward shock wave (Prantzos 2012). But the full interpretation of these constraints still requires 
detailed time-dependent MHD models of the expansion of multiple shocks (winds and supernovae) produced with time and space densities representative of OB associations. CR activity in starburst superbubbles is further discussed in section 8.1 .

\section{$2.3 \quad$ Spectral puzzles}

2.3.1 Above GeV EnERgIEs Spectral measurements to high energies are restricted to the more abundant elements, but the new data from AMS-02, PAMELA, CREAM, ATIC-2, and TRACER (see Table 1) have revealed spectral deviations from simple power laws well below the knee energy.

The finding with PAMELA of a hardening of the proton and Helium spectra near $200 \mathrm{GeV} /$ nucleon has been the subject of much theoretical attention, but has not been confirmed by AMS-02. A powerful magnet, combined with long exposures on the International Space Station, gives the latter significant advantages over previous instruments. It shows with high accuracy that the proton and Helium spectra follow power laws without any break in the $0.1-1.8 \mathrm{TeV}$ band (see Fig. 1). The Helium spectrum smoothly extends with index 2.65 (in particle flux per momentum unit) up to $50 \mathrm{TeV}$. In constrast, the CREAM and ATIC-2 data require a small upturn in protons above a few $\mathrm{TeV}$, with a change in index from $2.792 \pm 0.002$ in the $0.1-1.8 \mathrm{TeV}$ band (AMS-02) to $2.728 \pm 0.018$ above $2 \mathrm{TeV}$ (CREAM).

Another essential feature is the spectral difference between protons and Helium, first measured with PAMELA, and then confirmed by AMS-02 (see Fig. 1). The $\mathrm{He} / \mathrm{p}$ ratio increases by more than a factor of 2 from $20 \mathrm{GeV}$ to $50 \mathrm{TeV}$.

The CREAM and TRACER experiments have obtained consistent spectra for all major nuclei from Boron to Iron, up to a few $\mathrm{TeV} /$ nucleon. These spectra, with indices nearly equal at $2.66 \pm 0.04$, are consistent with the Helium one. An exception is Nitrogen, which shows a flattening beyond $100 \mathrm{GeV} /$ nucleon.

The spectral hardening in protons challenges the current propagation models. Potential causes under investigation include the manifestation of new types of CR sources to complement SNRs, spatial variations in diffusion properties, local anomalies due to nearby sources or to different transport in the Local Bubble, spallation effects, etc. The change in $\mathrm{He} / \mathrm{p}$ ratio is rather interpreted in terms of acceleration processes (different acceleration efficiencies or injection efficiencies into the shock, chemical variations in the upstream medium, activity of the reverse shock, etc.). We point the reader to the introductions of Vladimirov et al. (2012) and Ptuskin, Zirakashvili \& Seo (2013) for further literature on both spectral dilemmas. More data on different nuclei are expected soon from AMS-02. They should allow these scenarios to be tested with unprecedented accuracy.

2.3.2 Below $2 \mathrm{GEV}$ At low energies, a monumental event has been the arrival of Voyager 1 near the heliospheric boundary at 122 A.U., on August 25, 2012, and the simultaneous detection of an abrupt increase in CR flux at all kinetic energies below $\sim 1 \mathrm{GeV}$ (Stone et al. 2013). Whether CRs have fully unimpeded access to Voyager 1, free of solar modulation and of local interstellar modulation, is still under debate (Stone et al. 2013). If so, these data allow the interstellar CR spectrum to be sampled directly for the first time, down to the kinetic energies below $10 \mathrm{MeV}$ that are essential to estimate the level of CRinduced ionization in the ISM (see Sect. 5 and Fig. 7 of Padovani, Galli \& Glassgold 2009). The differential spectra of protons and Helium appear to be 
rather flat below kinetic energies of $100 \mathrm{MeV}$ per nucleon (see Fig. 1). We defer to Sect 4.1 the comparison with the proton spectrum inferred in the nearby ISM from $\gamma$-ray observations, and its implications.

Voyager 1 has also measured a stable $\mathrm{He} / \mathrm{p}$ ratio close to solar abundance up to hundreds of $\mathrm{MeV}$ in kinetic energy, at variance with the 1.5 solar value found at $\mathrm{TeV}$ energies. We look forward to Voyager 2, which is expected to leave the heliosphere in a few years, to provide further and independent data at low energy.

2.3.3 CRs INSIDE THE HELIOSPHERE We shortly digress from direct measurements to remote $\gamma$-ray measurements to probe CRs in the region between the Earth and the Sun. The possibility of detectable $\gamma$-rays from CR electrons upscattering sunlight or star light was pointed out by Moskalenko, Porter \& Digel (2006) and Orlando \& Strong (2007), and confirmed with CGRO-EGRET data (Orlando \& Strong 2008). This emission produces a diffuse "halo" of $\gamma$ rays, many degrees in extent, centred on the Sun. The spectrum and angular profile can be measured to follow the particle evolution. The quiescent solar disc is also a $\gamma$-ray source as CR nuclei interact with the photospheric gas. Continuous monitoring of these emissions on a daily basis with Fermi-LAT enables systematic studies of solar modulation through the solar cycle, and of CR transport well within the orbit of Mercury, which is otherwise hardly possible (Abdo et al. 2011).

This extended emission constitutes a significant $\gamma$-ray foreground which has to be accounted for, and removed, in studies of diffuse $\gamma$ rays from the Galaxy and beyond. In future, Fermi-LAT will deepen this study, and eventually detect such emission around nearby bright $\mathrm{O}$ and $\mathrm{B}$ stars, thereby opening a window onto their astrospheres.

\subsection{Positron excess at high energies}

The most surprising result of the past years was the discovery of a high-energy excess of positrons over that predicted from the decay of $\pi^{+}$pions produced in hadronic interactions between CR nuclei and interstellar gas in the Milky Way (Adriani et al. 2009). It has since been confirmed by AMS-02 and Fermi-LAT (see Table 1). In addition to its main goal as a $\gamma$-ray telescope, Fermi-LAT is indeed also an excellent lepton detector. It has helped extend the spectral measurements to $1 \mathrm{TeV}$ for total leptons and to $200 \mathrm{GeV}$ for separate charges, with the use of the Earth's magnetic field to separate them. The ground-based Cherenkov telescope H.E.S.S. also measures leptons, though not distinguishing charge. The total lepton spectrum resulting from these instruments shows no sign of fine structure or peak at a few hundred $\mathrm{GeV}$ (invalidating the ATIC-2 detection), but exhibits signs of a cutoff at $\mathrm{TeV}$ energies, as expected from the severe radiative losses incurred by these particles during propagation.

The positron fraction is seen to rise from $0.05 \%$ near $10 \mathrm{GeV}$ to about $0.15 \%$ above $200 \mathrm{GeV}$ where it starts to flatten in the AMS-02 data. The rise is due to an increase in positron flux rather than a deficit in electrons. The excess suggests a primary positron source, for example pulsars or pulsar wind nebulae, although much attention has also been devoted to origins in dark matter annihilation and decay. Different propagation histories between electrons and positrons may also induce an asymmetry. CR positrons and protons of the same rigidity are scattered by the same Alfven waves, but not the electrons. Since there are more waves generated by the numerous CR protons than by the sparse CR electrons, positrons may diffuse more efficiently and have a longer residence time in the 
Galaxy than electrons (Blandford, Simeon \& Yuan 2014).

\section{Advances in cosmic-ray propagation}

Primary CR nuclei produce secondary nuclei by spallation in the ISM. The rate of secondary production can be calculated using the primary spectrum and spallation cross-sections, so that the secondary source function in the diffusion equation is in principle known. This is the key to using secondary/primary ratios to test CR propagation models and to constrain the amount of reacceleration during propagation. Secondaries include antiprotons and positrons as well. Precise antiproton measurements were made by BESS and PAMELA (see Table 1).

The most striking feature in the CR composition is the large abundance of spallation products like Lithium, Beryllium, Boron, and sub-Iron elements. These are absent or minor in the ISM, but have comparable abundances to their primary parents due to the large number of interactions between primaries and interstellar atoms during their Galactic journey (see Sect. 5.4).

The most useful ratio to probe CR propagation is Boron/Carbon (B/C), which is accurately measured by PAMELA, AMS-02, and TRACER up to $1 \mathrm{TeV}$ (see Table 1). Carbon is almost entirely primary, while Boron is entirely secondary, being a spallation product of Carbon, Oxygen, and heavier nuclei. Hence the evolution of $\mathrm{B} / \mathrm{C}$ with momentum $p$ primarily reveals the momentum-dependence of the diffusion coefficient $D_{x x}(p)$, somewhat independently of source properties since secondaries have nearly the same energy per nucleon as the parent. The measured decrease of $\mathrm{B} / \mathrm{C}$ with $p$ (see Fig. 3) indicates a decrease in the amount of matter traversed by the higher- $p$ primaries, thus an increase in $D_{x x}(p)$ with $p$. Representing $D_{x x}(p)$ as a power law, the index ranges from 0.3 to 0.8 depending on the model. This index explains the difference between the CR spectra escaping supernova shockwaves, with index near 2.2, and the steeper spectra observed in the ISM, with index near 2.7 after propagation. Fig. 3 shows examples of consistent modelling of the proton, Helium and B/C data from AMS-02 and PAMELA, with different diffusion assumptions.

Significant curvature is observed in the $\mathrm{B} / \mathrm{C}$ spectrum above $20 \mathrm{GeV}$. It can trace a change in $D_{x x}(p)$ between the Galactic disc and halo, due to different levels of MHD turbulence (Evoli \& Yan 2014), or a change from CR diffusion on self-generated Alfven waves (generated by streaming instability) to diffusion on ambient turbulence in the ISM (Aloisio \& Blasi 2013). It can also trace effects within CR sources, such as reacceleration of Galactic CRs passing by, or spallation reactions inside SNRs, hence probing the average CR residence time and gas density inside accelerating remnants (Berezhko et al. 2003).

Radioactive secondaries like ${ }^{10} \mathrm{Be}$, with its 1.6 Myr rest-frame half-life, are sensitive to the time spent in the Galaxy. The combination of stable and radioactive secondaries allows the confinement time, thus the size of the CR halo, to be estimated. The measurement of ${ }^{10} \mathrm{Be} /{ }^{9} \mathrm{Be}$ up to $2 \mathrm{GeV}$ with ISOMAX (Hams et al. 2004) tends to be higher than predictions from current propagation models, but it has unfortunately not yet been repeated, the second flight being unsuccessful. Results from new experiments would be very valuable. The scale-height of the CR halo can be constrained by the ${ }^{10} \mathrm{Be} /{ }^{9} \mathrm{Be}$ ratio but is still uncertain (Putze, Derome \& Maurin 2010; Strong, Moskalenko \& Ptuskin 2007; Trotta et al. 2011). Alternatively, the scale-height can be constrained by propagation analyses of elec- 
tron/positron spectra in the CR together with synchrotron emission (Di Bernardo et al. 2013).

3.0.1 PARAMETRIC STUdies OF CR PROPAGATION Systematic parametric studies of $\mathrm{CR}$ propagation models using the USINE code and a Bayesian Monte Carlo Markov Chain technique (Lavalle, Maurin \& Putze 2014; Maurin, Putze \& Derome 2010; Putze, Maurin \& Donato 2011) show that a wide range of momentum-dependence of $D_{x x}(p)$ can fit the B/C data, in part because they include convection which allows a larger range of models. They also show that spectral differences between primaries can be naturally ascribed to transport effects, and that secondary positrons exclude a very steep variation of $D_{x x}(p)$, which was not a very likely scenario anyway. These analyses, and that of Trotta et al. (2011), illustrate the advantages of a Bayesian approach in constraining multi-parameter problems of this kind.

3.0.2 Diffusive REACCELERATION AND Alternatives Diffusive reacceleration is simply diffusion in momentum due to the gain and loss of momentum off moving scatterers; hence there is a basic relation between $D_{p p}$ and $D_{x x}$ (see e.g. Strong, Moskalenko \& Ptuskin 2007). Thornbury \& Drury (2014) give a useful and clear derivation of the reacceleration formula and also clarify its relation to the original Fermi second-order mechanism. This process has been frequently included in models since it explains the momentum-dependence of $\mathrm{B} / \mathrm{C}$ without an ad-hoc break in the momentum-dependence of $D_{x x}(p)$ and it is compatible with the $D_{x x}(p) \propto p^{1 / 3}$ variation expected for Kolmogorov turbulence. The question remains, however, of the actual importance of this process in the ISM. In the models with large reacceleration, a significant amount of energy is injected into CRs from the ISM itself, so that CR acceleration involves more than just the standard sources like SNRs. The source of this energy poses a problem and should be studied in more detail. As discussed in Section 7.2, reacceleration at the level often invoked seems incompatible with the low-frequency synchrotron spectrum, which is sensitive to the electron and positron spectra around a few $\mathrm{GeV}$. Secondary antiprotons provide another test of reacceleration. Models which fit $\mathrm{B} / \mathrm{C}$ underpredict antiprotons below a few $\mathrm{GeV}$.

On the other hand, diffusion models without reacceleration have a problem to reproduce the B/C spectrum without either a very large break in $D_{x x}(p)$ (Strong \& Moskalenko 1998), an additional velocity dependence in $D_{x x}(p)$, or dissipation of MHD waves (Ptuskin et al. 2006). But the use of B/C to constrain models depends critically on the level of solar modulation adopted to convert the modelled $\mathrm{B} / \mathrm{C}$ ratio (outside the heliopshere) to the observed one, since sub-GeV nuclei are strongly deccelerated in the heliosphere. A lower level of modulation than the value $\approx 500 \mathrm{MV}$ often adopted would ease the tension. Lave et al. (2013) use a modulation potential of $250 \mathrm{MV}$ to compare models with and without reacceleration to the $\mathrm{B} / \mathrm{C}$ data taken near the solar minimum.

Convection also plays a role in the sub-GeV/nucleon range, since it gives an energy-independent escape time. An interpretation of Voyager $1 \mathrm{CR}$ data as evidence for a Galactic wind, using a 1D transport model, has been put forward by Schlickeiser, Webber \& Kempf (2014).

3.0.3 SECONDARY PRODUCTION IN CR sources In the past, secondary quantities like B/C and the positron and antiproton spectra have usually been assumed to be entirely produced in the ISM, and hence related solely to interstellar propagation. As noted above, some secondaries must, however, be produced 
in the CR sources before sprimaries escape, and this produces an additional flux which complicates the picture. Berezhko \& Ksenofontov (2014) calculate antiproton and Boron productions in SNR and compare them with PAMELA and AMS-02 data. Similarly, Mertsch \& Sarkar (2014) compute secondary positron production in nearby SNR, and relate this to secondary Boron and antiproton production which can be tested with future data.

\subsection{Advances in propagation physics}

An important development has been to compute explicitly the trajectories of charged particles in the magnetised ISM, thus going beyond the analytical approximations of e.g. the quasi-linear theory. This involves the Lorentz force and Maxwell's equations and can address issues like cross-field diffusion, momentumdependence, etc. This approach was pioneered by Giacalone \& Jokipii (1994, 1999). The turbulent magnetic field is modelled as a superposition of modes with a power-law spectrum, using Alfven waves (i.e. time-dependent), in addition to the regular field. The trajectories are computed for large numbers of particles, and then statistical properties - diffusion tensors etc - are calculated. The diffusion coefficient perpendicular to the magnetic field was found to be much smaller than the parallel one. Casse, Lemoine \& Pelletier (2002) extended this approach to higher energies and a wide range of environments, including SNR, superbubbles and radio-galaxies. Lazarian \& Yan (2014) found that superdiffusion - faster than classical diffusion because of field-line divergence - can be important in CR source regions.

3.1.1 CR PENETRATion into MOlecular ClOuds Fatuzzo et al. (2010) and Fatuzzo \& Melia (2014) have performed numerical experiments using particle trajectories in various phases of the ISM with MHD turbulence. Their goal was to obtain scaling relations for the diffusion coefficient $D_{x x}(p)$ in a variety of environments in the Galaxy and in CR sources. They have found significant deviations from the results of quasi-linear theory for strong turbulence. They have studied spatial diffusion into molecular clouds and the possibility of particle acceleration by diffusion in energy in these clouds, concluding that it is probably not very important.

Observations have indicated that CRs with energies above a $\mathrm{GeV}$ have diffusion lengths exceeding typical cloud dimensions (Abdo et al. 2010g, Ackermann et al. 2011b, Hunter et al. 1997), but various mechanisms can alter their flux inside the dense molecular phase. This is particularly true at energies well below a $\mathrm{GeV}$ that are invisible to $\gamma$-ray observations, but essential to gas ionization and chemistry.

Exclusion processes include resonant scattering on magnetic irregularities generated by the CRs themselves (excited by their streaming), for wavelengths commensurate with their gyroradii ( $\ll \mathrm{mpc})$. However, maintaining the Alfven waves against ion friction with the predominant neutrals in the clouds requires too large a power (Cesarsky \& Volk 1978). Another depletion scenario is triggered by the increased ionization losses in dense gas, which leads to a net CR streaming flux inward (and electron flux outward), which in turn generates Alfven waves on the field lines connecting the cloud edges to the intercloud medium. These waves impede $\mathrm{CR}$ penetration at energies below a $\mathrm{GeV}$ and suppress their flux by several orders of magnitude at tens of MeV (Cesarsky \& Volk 1978, Skilling \& Strong 1976), but at GeV energies, Everett \& Zweibel (2011) predict only a 7.5\% de- 
crease in CR pressure at a rather extreme transition between the warm, diffuse, ionized medium and a dense, cold atomic or molecular cloud.

The overall magnetic structure of sub-parsec cores also leads to CR exclusion (magnetic mirroring) or concentration (magnetic focussing). The former dominates in a large variety of core configurations and it adds a depletion factor of 2 to 4 to the density of ionizing CRs (Padovani \& Galli 2011). In collapsing pre-stellar cores, the ionization rate can fall well below $10^{-18} \mathrm{~s}^{-1}$ in the central condensation because of magnetic mirroring (Padovani, Hennebelle \& Galli 2013).

Conversely, CR trapping in the magnetic bottles created between dense cores may locally increase the $\gamma$-ray emissivity per gas nucleon, $q_{\gamma \mathrm{H}}$, if the trapped particles substantially lose their energy before being replenished by diffusion. A 3 - to 5 -fold increase in $\gamma$-ray emissivity has been predicted (Cesarsky \& Volk 1978), but numerical simulations indicate that $\mathrm{TeV}$ particles effectively scatter off magnetic turbulence and that they smoothly diffuse throughout the complex uniform+turbulent field of a molecular cloud (Fatuzzo et al. 2010).

For energies above a $\mathrm{GeV}$, all these concentration/exclusion processes leave an energy-dependent signature that is not detected in the present $\gamma$-ray data, thereby supporting the use of hadronic interactions between CRs and interstellar gas, and the subsequent $\gamma$-ray emission, to trace all forms of gas in the ISM.

\subsection{Advances in propagation models}

Numerical models of CR propagation are an important tool to enable prediction of a wide range of observables to be compared with experiments. The original large project of this kind, GALPROP, is maintained as public software ${ }^{3}$, with large number of users and publications based on it. Other projects with similar goals have been developed, in particular DRAGON (Evoli \& Yan 2014, Gaggero et al. 2014, and references therein), which extends the CR propagation to anisotropic and spatially-dependent diffusion, and to diffusion properties related to the level of interstellar MHD turbulence. DRAGON is also publicly available ${ }^{4}$. Examples of DRAGON predictions for nuclei and leptons are shown in Figs. 2 and 3.

USINE (Putze, Maurin \& Donato 2011, and references therein) is a semianalytical CR-propagation package, which has the advantage of speed for model parameter explorations, and which has served many recent investigations.

These numerical packages have limitations in terms of both accuracy and speed, and hence the spatial resolution achievable. Their use has often been restricted to $2 \mathrm{D}$ models with cylindrical symmetry. The new PICARD code (Kissmann 2014, Werner et al. 2013, 2014) is fully 3D in concept, and has state-of-the art numerical techniques. It can handle models with spiral structure at good (e.g. $10 \mathrm{pc}$ ) resolution with reasonable computer resources.

In future, more detailed physical content is needed in these models, including Galactic winds, treatment of the boundary conditions, and the connection to the extragalactic medium. Convection in the form of Galactic winds is supported by recent X-ray observations (Everett et al. 2012), so it will be important to include their effect. A Galactic wind driven by CR streaming was studied by Uhlig et al. (2012).

GALPROP and similar programs are still basically phenomenological, i.e. they

\footnotetext{
${ }^{3}$ see http://galprop.stanford.edu and http://sourceforge.net/projects/galprop. For details of the developments see http://www.mpe.mpg.de/ aws/galprop_explanatory_supplement.pdf.

${ }^{4}$ at http://www.dragonproject.org
} 
use diffusion and convection as parameterized processes, but do not treat these at the physical level. They also treat CRs as test particles, and do not include their dynamical effects on the ISM. A different approach, in which the influence of CRs on the Galaxy is treated as a CR relativistic fluid in an MHD treatment, has been used to study a CR-driven dynamo generating the Galactic magnetic field (Hanasz et al. 2009, 2012b; Wóltański, Hanasz \& Kowalik 2013). This uses the PIERNICK program (Hanasz et al. 2010, 2012a). It can be extended in future to include the energy spectrum of CRs instead of a single fluid, to enable comparisons with the various observational diagnostics as for the other approaches. On smaller scales (100 pc), Girichidis et al. (2014) have studied the dynamical impact of CRs escaping from a SNR on the surrounding ISM using the MHD code FLASH; the propagation is anisotropic, and the surrounding gas is accelerated.

The study of CR secondaries, so important for propagation studies, is still limited by the accuracy of nuclear cross-sections. An initiative to improve them is described by Moskalenko (2011). A new treatment of the complicated processes involving the light $(\mathrm{Z} \leq 2)$ nuclei is given in Coste et al. (2012), including for example Deuterium production by p-p fusion reactions. Antiproton production physics also requires improvements to take advantage of current and future data (Kappl \& Winkler 2014). Hadronic $\gamma$-ray and lepton production poses a challenge to our knowledge of the basic physics as well (Dermer et al. 2013a).

\section{Cosmic-ray wanderers in the Milky Way}

Constraints on the propagation history of CR nuclei in and around the Galaxy are registered locally in their composition, and remotely in their interactions with interstellar gas, therefore in variations of the $\gamma$-ray emissivity rate per gas nucleon, $q_{\gamma \mathrm{H}}$. Abundance ratios between CR primaries and/or secondaries suggest that $10-\mathrm{GeV} /$ nucleon $\mathrm{CRs}$ spend most of their $10^{8}$-year residence time diffusing away from the thin Galactic disc, which they occasionally, but repeatedly, cross. By the end of their journey, they have traversed a gas column density $N_{\mathrm{H}} \approx 3 \times 10^{24} \mathrm{~cm}^{-2}$, which largely exceeds the disc-through integrals of $(0.1-$ 1.4) $\times 10^{21} \mathrm{~cm}^{-2}$ characterizing the ISM to a radius of $20 \mathrm{kpc}$ (Kalberla \& Dedes 2008, Pineda et al. 2013). These abundance ratios set the magnitude and momentum dependence of the diffusion coefficient, yet one should recall several caveats:

- The observational degeneracy between the momentum-dependence of $D_{x x}(p)$ and the particle spectra emerging from sources (Coste et al. 2012; Putze, Maurin \& Donato 2011). This degeneracy is somewhat alleviated by the observation of secondary-to-primary ratios.

- The possibly strong environmental effects on the diffusion properties via the level of interstellar MHD turbulence and, in particular, of fast magnetosonic modes that efficiently scatter CRs (Yan \& Lazarian 2008). They imply different diffusions through the Galactic disc and halo (Evoli \& Yan 2014), hence also in the different ISM phases.

- The local character of the CR sample reaching the Earth, with more than half of the light/heavy nuclei produced within 2/1 kpc (Taillet \& Maurin 2003).

- the specificity of the local ISM with its large cavities open to the halo 
(Galeazzi et al. 2014, Lallement et al. 2014) and surrounded by the Gould Belt clouds that have formed stars at an enhanced rate over the past 60 Myr (Grenier 2000, Perrot \& Grenier 2003). CR sources from the Gould Belt can induce significant fluctuations in the $\mathrm{CR}$ abundance ratios near the Sun (e.g. by $\sim 50 \%$ in B/C for durations $<0.5 \mathrm{Myr}$, Büsching et al. 2005). The hollow chimney can severely affect the relative abundance of heavy nuclei $(A \geq 50$, Combet et al. 2005). With the opposite effects of a lower spallation rate in the chimney and enhanced CR source production in the expanding Belt, together with potentially different diffusions in and out of the chimney, the net effect on abundance ratios is unclear. It definitely requires further modelling to improve the Galaxy-wide propagation models.

In this context, it is worth comparing the CR spectrum inferred from $\gamma$-ray observations in various locations around the Sun and to further distances in the Local and Perseus spiral arms, where the gas mass and kinematical separation along sightlines are best constrained.

It is important to probe the $\mathrm{CR}$ spectrum in the HI phase, rather than in the total gas, because of the more robust $N_{\mathrm{H}}$ determination in the Hi than in molecular gas, and because Hi dominates the surface mass density of the gas at the solar circle and beyond (Pineda et al. 2013). In the diffuse HI, where corrections for self absorption of the $21-\mathrm{cm}$ lines are small, the $\gamma$-ray emissivity $q_{\gamma \mathrm{H}}$ has been verified to scale linearly with $N_{\mathrm{HI}}$ across the $(0.1-2) \times 10^{21} \mathrm{~cm}^{-2}$ range (Abdo et al. 2009). In more massive clouds, $q_{\gamma \mathrm{H}}$ values are subject to 10-40\% uncertainties because of the Hi optical depth.

\subsection{Cosmic rays in the local ISM}

Analyses of the Fermi LAT $\gamma$-ray data have revealed a fairly uniform distribution of $q_{\gamma \mathrm{H}}$ emissivities in the Gould Belt and in the Local Arm (Abdo et al. 2009, 2010g, Ackermann et al. 2012b,d,f, 2011b, Casandjian 2012). The emissivity spectra displayed in Fig. 4 show less than $30 \%$ variations in amplitude and no spectral change across the $\gamma$-ray band, which samples CR energies of $0.5-10^{3}$ $\mathrm{GeV}$. The variations are commensurate with the uncertainties in $N_{\mathrm{HI}}$ and in the LAT exposure (Ackermann et al. 2012a).

The uniformity is remarkable given the large span in mass of the sampled clouds, from $10^{3}$ to $8 \times 10^{6} \mathrm{M}_{\odot}$, and given their notable differences in star-formation

activity, from the quiescent Cepheus and Polaris clouds to the starburst region of Cygnus. The same emissivity is also found in an intermediate-velocity cloud (IVA in Fig. 4), which appears to be sheared in space and in velocity, at a possible altitude of $60 \mathrm{pc}$ above the Galactic plane (Planck et al. 2014). Consistent values are found in three independent sectors along a 3-kpc long segment of the Local Arm . They compare well with the average obtained locally in thinner Hi gas at medium latitudes.

The observation of a uniform $q_{\gamma \mathrm{H}}$ emissivity in the solar neighbourhood provides a reference for the local interstellar spectrum (LIS) of CRs well outside the heliosphere (Casandjian 2012, Dermer et al. 2013b, Casandjian et al. ICRC 2013). Figure 1 shows a preliminary proton spectrum inferred from the local $q_{\gamma \mathrm{H}}$ measurement. Uncertainties in the hadronic production cross sections still limit its precision to within $30 \%$, but the data provide clear experimental evidence for solar modulation below tens of $\mathrm{GeV}$, and for good consistency between the CR spectra in and out of the solar system at high energy. 
There are preliminary indications of a break in the $\gamma$-ray inferred LIS below a few $\mathrm{GeV}$ in momentum. It is compatible with propagation effects and supported by the data taken by Voyager 1 near the heliopause. The Voyager data in fact suggest a pronounced turnover in momentum spectrum near a $\mathrm{GeV}$, i.e. a rather flat particle differential spectrum for kinetic energies below $100 \mathrm{MeV}$ (see Fig. 1). On the one hand, the CRs detected by Voyager 1 may still be decelerated by solar modulation and the true LIS may be above these data points; the question is still open (see the discussion in Stone et al. 2013). On the other hand, the $\gamma$-ray LIS extraction at low momentum is hampered by the uncertain subtraction of electron radiation in $q_{\gamma \mathrm{H}}$ at low $\gamma$-ray energies, and the break could be more pronounced. Voyager 1 has indeed detected a larger electron flux locally than that used to derive the broken power law shown in Fig. 1. Below the break, models predict a falloff due to ionization losses that cannot be probed in $\gamma$ rays, but the value of the break momentum itself bears information on adiabatic losses from a local Galactic wind and on the inhomoegeneity of the CR source distribution in the solar vicinity (Schlickeiser, Webber \& Kempf 2014).

The Voyager 1 data represent either the actual sub-GeV LIS, or a lower limit to it. Conversely, the $\gamma$-inferred LIS yields an upper limit to the sub-GeV LIS. Together they bracket the span of low-energy CRs more tightly than the test cases studied to estimate the $\mathrm{CR}$ ionization rate, $\zeta_{\mathrm{CR}}^{\mathrm{H}}$, of $\mathrm{H}$ atoms in the local ISM (Padovani, Galli \& Glassgold 2009; Rimmer et al. 2012). We have used the new data of Fig. 1 to calculate $\zeta_{\mathrm{CR}}^{\mathrm{H}}$ and the local CR energy density. To do so, we have extrapolated the Voyager 1 data down to $1 \mathrm{MeV}$ and we have used the ionization cross section of Spitzer \& Tomasko (1968) and solar modulation potentials of 450-500 MV for Pamela (Adriani et al. 2011) and 400 MV for AMS02. The latter was set to ensure compatibility of the demodulated spectrum with the $\gamma$-inferred LIS. Accounting separately for the CR protons and He spectra, and adding the contribution from heavier CR nuclei according to their fractional abundance and charge and/or mass (Padovani, Galli \& Glassgold 2009), we obtain $\zeta_{\mathrm{CR}}^{\mathrm{H}} \approx 1.4 \times 10^{-17} \mathrm{~s}^{-1}$ and a CR energy density of $1.9 \mathrm{eV} \mathrm{cm}^{-3}$. Using the same proton spectrum at high energy, but the extrapolation of the $\gamma$-inferred LIS down to $1 \mathrm{MeV}$, and scaling for the contribution of heavier nuclei, we find an upper limit of $2.8 \times 10^{-14} \mathrm{~s}^{-1}$ to $\zeta_{\mathrm{CR}}^{\mathrm{H}}$. This corresponds to a $\mathrm{CR}$ energy density so much larger than those of the other interstellar components (gas, magnetic field, starlight) that its consequences should be investigated.

A low sub-GeV CR spectrum, close to the Voyager 1 data, implies a rather mild decline in ionization rate with increasing depth inside the nearby clouds (Padovani, Galli \& Glassgold 2009; Rimmer et al. 2012). It also implies an ionization rate that is interestingly an order of magnitude below those derived from the bulk of the Galactic measurements of molecular ions in the diffuse ISM, even though the Sun lies in a diffuse region where low-energy CRs should not be severely depleted (see Indriolo 2012, for the $\zeta_{\mathrm{CR}}^{\mathrm{H}_{2}}$ distribution deduced from $\mathrm{H}_{3}^{+}$absorption lines, with $\zeta_{\mathrm{CR}}^{\mathrm{H}} \approx 0.65 \zeta_{\mathrm{CR}}^{\mathrm{H}_{2}}$ ). Locally, the $\zeta_{\mathrm{CR}}^{\mathrm{H}}$ upper limits of $(2-12) \times 10^{-17} \mathrm{~s}^{-1}$ obtained toward 100-200 pc distant stars in Sco-Oph compare well with the estimate based on Voyager data. But toward the more distant Perseus OB2 cluster (300 pc), the parallel observation of ten times larger ionization rates and of an almost uniform flux of super-GeV CRs over this zone (as seen in $\gamma$ rays) betrays strong environmental effects in the spatial distribution of the lowest-energy CRs. 


\subsection{Cosmic rays in the outer Galaxy}

The surface density of potential CR sources is expected to rapidly decline beyond the solar circle, along with the star-formation rate (Urquhart et al. 2014). We therefore expect a downward gradient in CR density as we move out in Galactic radius. However, the Fermi LAT data displayed in Fig. 4 show that the $\gamma$-ray emissivity per gas nucleon, $q_{\gamma \mathrm{H}}$, decreases by only $20 \%$ to $40 \%$ from the Local to the outer spiral arms, with no significant spectral change. These values readily confirm the long-standing "CR gradient problem", first raised by $C O S-B$ observations (Strong et al. 1988), then with CGRO-EGRET (Digel et al. 2001): the shallow $q_{\gamma \mathrm{H}}$ gradient in the outer Galaxy is barely consistent with the largest halo size of $\sim 10 \mathrm{kpc}$ allowed by the ${ }^{10} \mathrm{Be} /{ }^{9} \mathrm{Be}$ data for a uniform diffusion coefficient and for a CR-source distribution peaking in the inner Galaxy as expected for supernova remnants or pulsars (see Fig. 4).

Furthermore, the contrast in $q_{\gamma \mathrm{H}}$ between the Local or Perseus arms and their interregion is less than 10-20\% despite different interstellar and stellar environments. The correspondence in Fig. 4 between the $q_{\gamma \mathrm{H}}$ profile and that of the surface density of the gas mass wrongly suggests a linear coupling between the $\mathrm{CR}$ and ISM densities because the azimuthal averaging does not respect the spiral structure of the Galaxy. Such a coupling has been modelled (Hunter et al. 1997) and rejected by the data; it yields for instance a twice larger emissivity than actually measured in the $14-16 \mathrm{kpc}$ range.

In order to mitigate the gradient problem, Evoli et al. (2012) have proposed a strong, almost linear, coupling between the CR-source surface density and the diffusion coefficient perpendicular to the regular magnetic field. It is physically motivated by the enhanced turbulence level induced by stellar and supernova activity, which scatters CRs off the spiral arms and smooths out the radial distribution. It will be challenging, but instructive, to test this scenario at closer range against the lack of contrast in $q_{\gamma \mathrm{H}}$ (in magnitude and spectrum) in and out of the well-known Local and Perseus arms.

An alternative or complementary solution to the gradient problem is to look for missing gas in the inventory of the outer Galaxy since the $\gamma$ rays trace the product of the CR and gas densities. Cold Hi in the CNM is more prone to self absorption than diffuse warm Hi. From 8.5 to $10 \mathrm{kpc}$ in radius, the twofold $d r o p$ in CNM fraction cannot flatten the $q_{\gamma \mathrm{H}}$ profile. Beyond $10 \mathrm{kpc}$, the $15-20 \% \mathrm{CNM}$ fraction is rather uniform (Dickey et al. 2009, Pineda et al. 2013) and it should not strongly bias the measurement of an outward $q_{\gamma \mathrm{H}}$ gradient when averaging data over large regions.

On the other hand, large quantities of gas escape radio surveys at the darkneutral-medium (DNM) interface between the HI-bright and CO-bright media (see section 7.1). The abundance of missing gas exceeds that of the CO-bright $\mathrm{H}_{2}$ beyond the solar circle (Pineda et al. 2013, Planck Collaboration et al. 2011a). The $\gamma$-ray analyses include a joint $\gamma$-ray+dust map to account for this missing DNM gas, but only as integrals along sightlines and with a downward bias since the dust-to-gas ratio is known to decrease radially outward with metallicity (Sandstrom et al. 2013). Future observations of other DNM tracers, such as $\mathrm{OH}, \mathrm{CH}$, or $\mathrm{C}^{+}$, will constrain the DNM mass to large radii, but they require much higher instrumental sensitivities than currently available. Stellar extinction and reddening from future deep surveys provide an alternative. It is indeed important to accurately quantify the gas mass in the outer arms to assess whether the mea- 
sured $q_{\gamma \mathrm{H}}$ emissivities truly reflect large CR fluxes to ionize and heat the outer ISM.

\subsection{Cosmic rays in the inner Galaxy}

Fermi LAT $\gamma$-ray maps cover the full sky with angular resolution varying from degrees to several arcminutes with increasing photon energy. They allow critical tests of global Galactic models of CR emission. Figure 5 shows a full-sky map obtained for energies above $1 \mathrm{GeV}$, which readily displays how $\mathrm{CR}$ emission dominates over other sources of $\gamma$ rays in the Galactic disc. It spectacularly illustrates the close synergy between the total ISM traced in $\gamma$ rays and in dust, thereby demonstrating the absence of large variations in CR density across the Galaxy (for $\mathrm{GeV}-\mathrm{TeV}$ nuclei), even toward the active inner regions.

The first notable interstellar result from Fermi-LAT was the finding that the excess observed with CGRO-EGRET in the GeV range above the expected interstellar emission was evidently an instrumental effect. Hence this aspect covered in earlier reviews (e.g. Strong, Moskalenko \& Ptuskin 2007) must be revised.

A representative $\gamma$-ray spectrum of the inner Galaxy is displayed from $20 \mathrm{keV}$ to $100 \mathrm{GeV}$ in Fig. 6 (see also Fig. 15 of Ackermann et al. 2012g), together with its decomposition into continuum and line emission components, positronium included (Bouchet et al. 2011, Strong 2011). The Fermi-LAT data show reasonable agreement with the model predictions, which are chosen to be consistent with locally-observed CR spectra. The most detailed analysis to date (Ackermann et al. 2012g) finds a global agreement between GALPROP models and the Fermi-LAT data, for both spectra in various sky regions, and for broad longitude and latitude distributions. On smaller scales, however, there are numerous regions at variance with the average particle distributions predicted

by a Galaxy-wide model such as GALPROP. There is also still some sign of a high-energy excess, but this awaits reanalysis with improved data reductions and increased statistics from the extended mission. Inverse Compton emission notably contributes at both ends of the Fermi-LAT range, which can be jointly used in the future to explore the soft radiation fields in the inner Galaxy. The emission in the 1-30 MeV range is above the predictions, perhaps due to a population of $\mathrm{MeV} \gamma$-ray sources. Future data from Fermi-LAT should probe at least the upper end of this important and unexplored range. Likewise, measurements with Fermi-LAT of the $q_{\gamma \mathrm{H}}$ emissivity profile toward the inner spiral arms will be obtained soon to test the relative importance of diffusion and advection in CR transport in the turbulent inner regions, and to test the presence of a Galactic wind in the central zone (Breitschwerdt, Dogiel \& Völk 2002; Zweibel 2013).

4.3.1 Populations of UnRESOlved $\gamma$-RAY sourCes Extended supernova remnants may contribute to the apparently diffuse $\gamma$-ray emission in the Galactic disc, and to the excess at GeV energies (Völk \& Berezhko 2013). They are often difficult to separate from their clumpy interstellar environment, even with detectable fluxes, because of the limited angular resolution of Fermi-LAT. Populations of faint, unresolved sources, below the detection threshold, may also contaminate the interstellar emission (Ackermann et al. 2013a, Strong 2007). It involves mainly young and recycled millisecond pulsars. Their contribution amounts to several percent of the total diffuse emission above $10 \mathrm{GeV}$, and this fraction is expected to increase with poorer angular resolution at lower energies. This contamination has been largely ignored in analyses up to now. It does not 
directly impact the measure of the ISM $\gamma$-ray emissivity, $q_{\gamma \mathrm{H}}$, which is based on a spatial correlation between the $\gamma$-ray signal and gas column densities, as long as the gas and contaminating source distributions do not strongly correlate in space. Middle-aged and old pulsars, which dominate the $\gamma$-ray pulsar population, are fortunately loosely connected to the gas structures. This condition is likely to fail in the directions tangent to the spiral arms and in the crowded regions around the Galactic centre.

4.3.2 The Fermi Bubbles and Galactic centre The most spectacular discovery in the area of extended emission was the so-called Fermi Bubbles ( $\mathrm{Su}$, Slatyer \& Finkbeiner 2010); this is arguably the most unexpected discovery of the Fermi mission so far. A detailed spatial and spectral analysis is presented in Ackermann et al. (2014). The bubbles are two $\sim 50^{\circ}$ diameter, oval regions of emission with sharp edges, roughly symmetrical with respect to the Galactic centre. They extend over several kpc toward the north and south Galactic poles, assuming a large distance as seems probable given their apparent relation to the Galactic centre and their axis perpendicular to the Galactic plane (the local ISM is dominated by the inclined geometry of the Gould Belt, Lallement et al. 2014, Perrot \& Grenier 2003). They have a much harder spectrum than the rest of the interstellar emission, hence they appear clearly in skymaps at energies above 10 $\mathrm{GeV}$ even without complex foreground modelling.

They partially overlap two giant radio lobes emanating from the Galactic bulge and permeated by large magnetic fields, up to $1.5 \mathrm{nT}$ (Carretti et al. 2013). These lobes transport $10^{48} \mathrm{~J}$ into the halo and are possibly driven by the Galactic wind evidenced through multi-wavelength observations of the central $200 \mathrm{pc}$ zone. The Fermi Bubbles are certainly filled with CRs with a radiative power of $2 \times 10^{30}$ $\mathrm{W}$ in $\gamma$ rays, but the hadronic or leptonic nature of the dominant particles is presently unknown.

Hence the bubbles have relevance to $\mathrm{CR}$ origin and propagation, but it will be a long time before their actual role becomes clear. CR pressure is necessary to launch a massive wind in the inner Galaxy (Zweibel 2013). The central supernova rate can sustain the large $\mathrm{CR}$ population required to power such a wind and to explain the non-thermal emissions observed in the region (Crocker et al. 2011), but with difficulties (Yoast-Hull, Gallagher \& Zweibel 2014). Observations include synchrotron ageing of CR electrons with increasing distance from the Galactic plane (Law 2010), and diffuse TeV emission detected by H.E.S.S. and attributed to pion decay, possibly from a transient source of CR protons near the Galactic centre (Aharonian et al. 2006). The data suggest that most CRs be advected away into the halo (Crocker et al. 2011). It is, however, unclear whether the Fermi Bubbles record the past starburst and wind activity of the central nucleus, or jet activity from its black-hole. Among the many models, Yang, Ruszkowski \& Zweibel (2013) made detailed simulations of a leptonic origin from recent AGN jet activity; Lacki (2014) proposed CR acceleration at starburst wind termination shocks, while a hadronic model with 100 Myr confinement has been suggested by Crocker et al. (2014). Past AGN activity has also been invoked (Barkov \& BoschRamon 2014). The presence of a powerful CR-driven wind would help explain the relatively small gradient observed in interstellar $\gamma$ rays toward the central zones (Breitschwerdt, Dogiel \& Völk 2002). Ultraviolet absorption spectroscopy has been used to trace the kinematics of gas associated with the Fermi Bubbles, which can be interpreted in terms of a biconical outflow at velocities exceeding 
$900 \mathrm{~km} \mathrm{~s}^{-1}$ (Fox et al. 2014). As for the AGN connection, we remark that the Fermi Bubbles bear a close resemblance to the lobes of the nearby Cen A radio galaxy, which have also been detected by Fermi-LAT (Abdo et al. 2010d).

The Galactic centre itself shows two bright coincident $\gamma$-ray sources, above 1 GeV with Fermi-LAT and up to $40 \mathrm{TeV}$ with H.E.S.S. (Chernyakova et al. 2011), with matching, but complex spectra, and whose origin is unclear at present. Possibilities include large numbers of pulsars (millisecond or normal, Yuan \& Zhang 2014), CR proton sources (Carlson \& Profumo 2014), lepton sources, and darkmatter models. The coincidence with the extended $511 \mathrm{keV}$ positron annihilation line source observed by INTEGRAL is intriguing; perhaps the sources are related in some way.

\subsection{Global properties of the high-energy Galaxy}

Figure 7 shows the luminosity spectrum of the Galaxy from radio to $\gamma$ rays, as well as proton and electron luminosities (Strong et al. 2010). This is what an extraterrestial, or rather an extragalactical colleague (e.g. in M31) would observe looking at our Galaxy. It illustrates that high-energy radiation is only a small fraction of the optical and infrared emission. It shows that hadronic and leptonic processes have comparable power, and that the leptonic emission is roughly equal in the synchrotron and $\gamma$-ray bands (inverse Compton emission). The Galaxy is a fair lepton calorimeter, with about half the energy escaping to intergalactic space, which is of interest in the context of the radio-far-infrared correlation observed in galaxies (see Sect. 8.4). In contrast, CR protons escape with only about $10 \%$ loss by hadronic interactions with gas. The total CR-related luminosity of the Galaxy is about $10^{41} \mathrm{erg} \mathrm{s}^{-1}$ (depending on the model adopted), consistent with the supernova rate, the energy released in their explosions, and a few percent acceleration efficiency.

\section{Cosmic-ray/ISM interaction processes}

Cosmic rays interact with interstellar matter in two distinct ways: through collective behaviour and by microscopic atomic, molecular, and nuclear processes. Collective interactions include those with magnetic fields and waves, and naturally yield to a fluid description at energies where the mean-free-path is very small compared with characteristic scales (Zweibel 2013). At the other extreme, CR particles collide one-by-one with interstellar atoms, molecules, and dust particles, and with the radiation field. In order to appreciate the effects of CRs on interstellar matter and to understand the indirect tracers of them, it is necessary to consider many processes over an enormous range of energy.

\subsection{Atomic and molecular processes}

Many aspects of interstellar matter require a microscopic, kinetic description, because the physical and chemical states of the gas and dust are far from thermodynamic equilbrium. Details can be found in recent textbooks, for example Draine (2011), Tielens (2005). Briefly, the most important coupling between CRs and interstellar matter is through ionization processes. Most of the Galactic interstellar mass is contained in the neutral phase and most of that is hydrogen, either in atomic $(\mathrm{H})$ or molecular $\left(\mathrm{H}_{2}\right)$ form. Columns of neutral hydrogen $N_{\mathrm{H}} \sim 10^{18}$ 
to $10^{21} \mathrm{~cm}^{-2}$ are sufficient to make the neutral interstellar matter opaque to ionizing radiation from the hottest, most luminous thermal sources. To first order, hydrogen-ionizing photons are confined to the immediate surroundings of their stellar sources in ionization-bounded, photoionized nebulae. Outside the nebulae, ultraviolet (UV) starlight with photon energies $h \nu<13.6 \mathrm{eV}$ can still penetrate the gas, ionizing trace elements with lower ionization potentials like $\mathrm{C}, \mathrm{S}, \mathrm{Si}, \mathrm{Mg}$, Fe, etc., until the column of associated dust particles grows thick enough to shield the gas completely. This radiation can also photodissociate molecules, although $\mathrm{H}_{2}$ and $\mathrm{CO}$ partly shield themselves even at small depths where dust particles are ineffective. Thus the fractional ionization of the neutral ISM is thought to vary from $x(e)=n(e) / n_{H} \sim 10^{-4}$ where carbon remains mostly ionized, decreasing with an increasing shielding column to $x(e)<10^{-7}$. A neutral region where thermal UV photons dominate the ionization, chemistry, and thermal state of the gas is called a photon-dominated region (PDR), which includes by definition the so-called diffuse and translucent molecular clouds (Snow \& McCall 2006).

Beyond the PDR, into the opaque zone of dark clouds and giant molecular clouds, it is thought that CRs control much of the ionization, chemistry, and thermal balance. The neutral interstellar matter will always retain some degree of ionization wherever CRs penetrate or wherever long-lived radioactive nuclei (notably ${ }^{40} \mathrm{~K}$ ) persist. If the residual ionization remains high enough, then the magnetic field can remain coupled to the gas over time scales long compared with the dynamical ages of clouds and filaments and thus remain important in the collapse to form stars; however, if the ionization becomes low enough, then the field can diffuse out of a cloud allowing it to collapse faster (Padovani, Hennebelle \& Galli 2013). The thermal state of the neutral gas is directly linked to ionization mechanisms, through which the liberated electrons transform atomic binding energy to kinetic energy in the gas. In PDR, photoelectric ejection from dust particles and very large molecules (e.g. polycyclic aromatic hydrocarbons, $\mathrm{PAH})$ is an important heating input.

$\mathrm{CR}$ nuclei and electrons ionize atoms and molecules in collisions. In the case of CR protons on hydrogen, the most important primary processes are:

$$
\mathrm{CR}+\mathrm{H} \rightarrow \mathrm{H}^{+}+e^{-}+\mathrm{CR} \text { and } \mathrm{CR}+\mathrm{H}_{2} \rightarrow \mathrm{H}_{2}^{+}+e^{-}+\mathrm{CR}
$$

in which the CR loses a negligible fraction of its energy and the liberated electron carries off a typical kinetic energy of $35 \mathrm{eV}$, almost independent of the CR energy. The CR barely feels this loss of energy and continues on its path. The electron is sufficiently energetic to ionize at least one additional atom or molecule, yielding further secondary electrons. The electron also engages in (a) inelastic collisions that excite bound states of hydrogen and helium and (b) elastic collisions with atoms, molecules, and thermal electrons. The competition among these processes depends upon the fractional ionization, molecular fraction, and helium abundance with different probabilities at each energy (Dalgarno, Yan \& Liu 1999) as the initial electron cascades from $35 \mathrm{eV}$ down to the mean thermal energy of the gas, $\sim k T \sim 8.6(T / 100 \mathrm{~K}) \mathrm{meV}$. That is, from the point of view of an ambient atom at $100 \mathrm{~K}$ temperature, even a $35 \mathrm{eV}$ electron is superthermal by a factor $>10^{3}$. The fraction of the initial electron energy that goes into heating is nearly $100 \%$ in fully ionized gas, but closer to $20 \%$ in neutral gas where secondary ionization and bound-state excitations prevail. Thus the heating rate by CRs in neutral gas is of the order of $10^{-27}\left(\zeta_{\mathrm{CR}} \mathrm{H} / 10^{-16}\right) \mathrm{ergs} \mathrm{s}^{-1}$ per hydrogen, where $\zeta_{\mathrm{CR}}^{\mathrm{H}}$ is the effective ionization rate $\left[\mathrm{s}^{-1}\right.$ per $\left.\mathrm{H}\right]$. A crucial point is that CRs can 
penetrate large columns of interstellar matter and ionize some hydrogen and helium in places that are well shielded from other sources of ionization like UV and X-ray photons. The ionization process provides enough heating to raise the gas temperature (lowest observed values near $8 \mathrm{~K}$ ) above that of the cosmic microwave background $(2.7 \mathrm{~K})$. CR ionization also yields enough ions to drive observable chemical activity through ion-neutral reactions that proceed rapidly even at temperatures below $10 \mathrm{~K}$.

CR Helium and heavier nuclei also ionize interstellar atoms and molecules, but at proportionally lower rates owing to their lower abundances in the CR spectrum. The processes, cross sections, and rates of all the important CR ionizing processes have been reviewed recently by Padovani, Galli \& Glassgold (2009, 2013).

\subsection{Cosmic-ray-induced ultraviolet radiation}

An electric discharge in $\mathrm{H}_{2}$ gas produces a characteristic UV emission spectrum at wavelengths $\lambda<200 \mathrm{~nm}$. Similarly, the secondary electrons from CR ionization of interstellar hydrogen can generate an internal source of UV radiation deep inside molecular clouds where starlight does not penetrate. The photochemical consequences of this UV radiation were first analyzed by Prasad \& Tarafdar (1983). The UV spectrum of $\mathrm{H}_{2}$ excited by electron-impact was modeled in more detail including the scattering effects of interstellar dust (Cecchi-Pestellini \& Aiello 1992; Gredel et al. 1989; Sternberg, Dalgarno \& Lepp 1987) and approximate expressions for the resulting photo-rates in the ISM have been incorporated into widely used chemical reaction networks (e.g. the UMIST database for astrochemistry $^{5}$ McElroy et al. (2013) and the Kinetic Database for Astrochemistry, $\mathrm{KIDA}^{6}$, Wakelam et al. 2012). There is still a need for more general descriptions of the spectra of CR-induced photons that are based upon the basic cross sections (Abgrall et al. 1997, Aguilar et al. 2008, Jonin et al. 2000), especially now that the electron-impact UV emission spectrum of $\mathrm{H}_{2}$ can be identified directly in some circumstellar disks (Ingleby et al. 2009).

Most interstellar molecules are photodissociated mainly by continuous absorption processes, in which case the dissociation rates due to CR-induced UV radiation scale linearly with the $\mathrm{CR}$ ionization rate of $\mathrm{H}_{2}, \zeta_{\mathrm{CR}}^{\mathrm{H}_{2}}$, and depend on an average probability that the photons are scattered or absorbed by dust particles. There are three molecules, $\mathrm{H}_{2}, \mathrm{CO}$, and $\mathrm{N}_{2}$, which are photodissociated at wavelengths $\lambda>91.2 \mathrm{~nm}$ only via narrow line absorptions rather than continuous processes. Thus the CR-induced photorates for $\mathrm{CO}$ and $\mathrm{N}_{2}$ are extremely sensitive to accidental coincidences in line wavelengths: extensive laboratory data and detailed calculations are needed to determine the rates. For example, when $\zeta_{\mathrm{CR}}^{\mathrm{H}_{2}}$ $=10^{-16} \mathrm{~s}^{-1}$, the CR-induced photodissociation rate of $\mathrm{N}_{2}$ is $\approx 10^{-15} \mathrm{~s}^{-1}$, while the rate for the ${ }^{14} \mathrm{~N}^{15} \mathrm{~N}$ isotopologue is quite different (Heays et al. 2014). This is one of the processes that can enrich molecular nitrogen in the heavier isotope in space. Such isotope fractionation can propagate through chemical networks to other-more easily observable - molecules.

\footnotetext{
${ }^{5}$ http://www.udfa.net

${ }^{6}$ http://kida.obs.u-bordeaux1.fr
} 


\subsection{Interaction with solid particles}

Interstellar dust particles make up approximately one per cent by mass of the total interstellar matter. The particles responsible for extinction of starlight (with diameters of the order of $0.1 \mu \mathrm{m}$ ) have refractory cores composed of amorphous carbon, silicates, and other materials. These particles present an average geometrical cross section of $\sim 10^{-21} \mathrm{~cm}^{2}$ per interstellar hydrogen. Where the particles are cold, $T_{\text {dust }}<20 \mathrm{~K}$, the abundant volatile species in the gas $(\mathrm{C}, \mathrm{N}, \mathrm{O}$, and their molecular forms, $\mathrm{H}_{2} \mathrm{O}, \mathrm{NH}_{3}, \mathrm{CO}$, etc.) freeze out onto the solid surfaces. Chemical reactions occur in these icy mantles, and the chemical processing can be enhanced by exposure to electromagnetic radiation, thermal changes, and particle bombardment. CR protons of energy $\geq 1 \mathrm{MeV}$ have a range in amorphous carbon of $\geq 2.8 \times 10^{-3} \mathrm{~g} \mathrm{~cm}^{-2}$; therefore, all such CRs pass through a typical interstellar dust grain without losing significant energy. From the perspective of the dust particle, however, the amount of energy deposited in a CR event is substantial. The possible effects of CRs on interstellar dust and ice mantles have been investigated in laboratory experiments at low energies, $1 \mathrm{MeV} /$ nucleon or less (Palumbo et al. 2008, e.g.), and more recently at energies of the CRs that also ionize gaseous hydrogen in the ISM (Dartois et al. 2013, Seperuelo Duarte et al. 2009). However, it may be difficult to distinguish effects of 1-100 MeV nuclei from those of UV photons (Gerakines, Moore \& Hudson 2001; Muñoz Caro et al. 2014). More importantly, the direct CR interactions with ice mantles tend to be less important than photo-desorption and photo-processing caused by the CR induced UV radiation described in the preceding subsection (Shen et al. 2004). Particle bombardment is expected to reduce the porosity of water ice, for example, consistent with the infrared absorption spectra of interstellar ices that indicate a general lack of highly porous solid water (Dartois et al. 2013).

\subsection{Spallation and synthesis of light elements}

Abundances of the isotopes of the light elements, Li, Be, and B, are especially important manifestations of $\mathrm{CR}$ interactions with interstellar matter. Because these nuclei undergo fusion reactions at relatively low temperatures inside stars, they tend to be destroyed rather than formed in stellar nucleosynthesis. Thus their abundances in interstellar matter are partly controlled by spallation reactions in which Galactic CR protons and $\alpha$ particles on $\mathrm{C}, \mathrm{N}$, and $\mathrm{O}$ nuclei yield ${ }^{6} \mathrm{Li},{ }^{7} \mathrm{Li}$, ${ }^{9} \mathrm{Be},{ }^{10} \mathrm{~B}$, and ${ }^{11} \mathrm{~B}$ in predictable amounts. There is competition from reverse spallation, where $\mathrm{C}, \mathrm{N}$, and $\mathrm{O}$ nuclei in the $\mathrm{CR}$ spectrum react with ambient hydrogen and helium, and also from neutrino-induced spallation in supernovae of type SNe II (Woosley et al. 1990) and possibly SNe Ic (Nakamura et al. 2010). The isotopes ${ }^{6} \mathrm{Li},{ }^{9} \mathrm{Be}$, and ${ }^{10} \mathrm{~B}$ are created mainly by $\mathrm{CR}$ spallation in the ISM. ${ }^{7} \mathrm{Li}$ comes from spallation and from $(\nu, \mathrm{He})$ in SNe, Big-Bang nucleosynthesis, stars on the Asymptotic Giant Branch (AGB), and novae. ${ }^{11} \mathrm{~B}$ comes from spallation and from $(\nu, \mathrm{C})$ in $\mathrm{SNe}, \mathrm{AGB}$ stars, and novae. Thus the isotope ratios in $\mathrm{Li}$ and $\mathrm{B}$ are governed not only by spallation rates, but also by the integrated history of stellar evolution, mass-loss, and mixing in the Galaxy.

In principle, the abundances of $\mathrm{Li}, \mathrm{Be}$, and $\mathrm{B}$ can be measured both in interstellar gas and in the Galactic CR spectrum (e.g., Adriani et al. 2014). With sufficiently accurate measurements of the light isotopes and of a progenitor nucleus ( $\mathrm{C}$ or $\mathrm{O}$, for example), it should be possible to determine the contributions 
of CR spallation, local variations in CR fluxes, and of diffusion to the production rates. In detail there are complications (see Sect. 3).

The ${ }^{7} \mathrm{Li} /{ }^{6} \mathrm{Li}$ abundance ratio can be measured directly in the ISM. For example, Knauth, Federman \& Lambert (2003); Knauth et al. (2000) found a ratio of $\approx 2$ in several diffuse clouds, a value which differs from that in the solar system (12.3) and which signifies recent lithium production from spallation. Observations of relatively low values of ${ }^{7} \mathrm{Li} /{ }^{6} \mathrm{Li}$ in interstellar matter near the supernova remnant IC 443 provide further evidence of recent lithium production by CR spallation and suggest that neutrino-induced spallation in the supernova event plays a minor role in lithium production (Taylor et al. 2012). Other measurements of the lithium isotope ratio are taken as evidence of homogeneous mixing and rapid recycling of the interstellar gas (Kawanomoto et al. 2009).

The abundance of boron and the ${ }^{10} \mathrm{~B} /{ }^{11} \mathrm{~B}$ ratio in interstellar gas have been studied (Howk, Sembach \& Savage 2000, Lambert et al. 1998, Ritchey et al. 2011). It is now possible to investigate abundances of the light elements and their isotopes in other galaxies. The Small Magellanic Cloud (SMC) has both lower abundances of interstellar C,N,O (Korn et al. 2000, Salmon et al. 2012) and lower CR fluxes than the Milky Way (Abdo et al. 2010f).If forward spallation dominated B production, its production rate in the SMC should be approximately 5\% that of the Milky Way (Brooks et al. 2002). The interstellar lithium abundance in the SMC (Howk et al. 2012) is consistent with expectations from Big Bang nucleosynthesis, thus placing constraints on the contributions of stellar nucleosynthesis and CR spallation in that low-metallicity system. Boron has now been detected in galaxies at high redshift $(z=2.0-2.6)$ via absorption spectroscopy of damped L $\alpha$ systems toward quasi-stellar objects (Berg et al. 2013, Prochaska, Howk \& Wolfe 2003). Reverse spallation (interstellar $\mathrm{H}$ and $\mathrm{He}$ as targets) might dominate in a metal-poor ISM, an effect that would be expected to increase with redshift. Interstellar beryllium has so far evaded detection (Hebrard et al. 1997).

\section{Cosmic-ray Stimuli on the ISM}

\subsection{In the vicinity of cosmic-ray sources}

Gamma-ray emission at GeV (Fermi-LAT) and TeV (H.E.S.S., MAGIC, VERITAS) energies has been associated with 17 prominent supernova remnants, with convincing, albeit not firm, observational evidence for in situ acceleration of CR nuclei in several instances. In parallel, acceleration by shock waves directly from the thermal gas has been explicitly demonstrated in the ab-initio simulations of MHD turbulence and ions by Spitkovsky (2008) for relativistic shocks (relevant to $\gamma$-ray bursts and active galactic nuclei) and by Gargaté \& Spitkovsky (2012) and Caprioli \& Spitkovsky (2014) for non-relativistic shocks, relevant to SNR. This seems to solve the 'injection problem' for CR acceleration, since it does not require the prior presence of supra-thermal particles. The acceleration efficiency is found to be 10-20\%, compatible with the energy requirements for a SNR origin of CRs. The fraction of thermal material accelerated to the non-thermal phase is of order $10^{-3}$. The energy spectrum in the non-thermal region exhibits a -2 spectral index, as in the standard theory of strong shock acceleration.

A few remnants among the $\gamma$-ray emitting ones are known to overtake surrounding clouds, which can serve as calorimeters to estimate the total energy in 
escaping CRs. The example of W28 and the TeV source H.E.S.S. J1801-233 is often quoted $^{7}$ (Hanabata et al. 2014). It is unclear how the slowing down of the shock wave in the dense cloud and the enhanced dissipation of Alfven waves due to the ion-neutral collisions in the dense gas actually impact the emerging CR and $\gamma$-ray spectra (Ellison \& Bykov 2011, Fang et al. 2013, Fang \& Zhang 2013). It is also possible that pre-existing Galactic CRs be reaccelerated by the slow shock in the crushed cloud, or that particles accelerated earlier in the SNR history remain trapped in the interaction region and produce pion-decay radiation even if shock acceleration is not efficient (Tang \& Chevalier 2014, Uchiyama et al. 2010). The potential detection of $\gamma$ rays from SNR-molecular-cloud associations has stimulated much new work on the early propagation of CRs near their sources (Malkov et al. 2013; Nava \& Gabici 2013; Ptuskin, Zirakashvili \& Plesser 2008; Telezhinsky, Dwarkadas \& Pohl 2012). Because of the gradient in CR density outside the SNR, the particles excite Alfven waves and their diffusion coefficient is both reduced below the standard ISM value, and anisotropic along and across the ambient regular magnetic field. It is still unclear how far the "enhanced CR cloud" extends and how long it survives to be detectable, but theoretical progress is rapid.

It is of interest to know how the CR spectrum extends in the crushed cloud below $100 \mathrm{MeV}$, where ionization and heating of interstellar matter is efficient. An increase in the CR-ionization rate above the Galactic average might be observable through enhanced abundances of molecules that are especially sensitive to the ionization rate. Ionization rates enhanced by as much as a factor of $10^{2}$ have been inferred in molecular gas near the $\gamma$-ray-emitting SNR W 51C (Ceccarelli et al. 2011) and W 28 (Vaupré et al. 2014), based mainly on observations of mm-wave emission lines of the molecular ions $\mathrm{HCO}^{+}$and $\mathrm{DCO}^{+}$. However, the abundance of the main isotopologue $\mathrm{H}^{12} \mathrm{C}^{16} \mathrm{O}^{+}$cannot be determined from saturated line intensities without good knowledge of the distribution of temperature and density in the emitting gas, nor can the $\mathrm{DCO}^{+} / \mathrm{HCO}^{+}$abundance ratio be used as a reliable measure of the electron fraction unless the temperaturedependent deuterium fractionation is well constrained. Hydrogen ions are more direct tracers of superthermal ionization. Indriolo et al. (2010) observed interstellar absorption of $\mathrm{H}_{3}^{+}$along six sightlines that pass through molecular material near the SNR IC 443 and deduced $\mathrm{H}_{2}$ ionization rates $\zeta_{\mathrm{CR}}^{\mathrm{H}_{2}} \approx 2 \times 10^{-15} \mathrm{~s}^{-1}$ in two cases.

Other probes to be investigated include $\mathrm{H}_{2}^{+}$that might be detectable at infrared and radio wavelengths (Becker et al. 2011) in extreme cases where the product of ionization rate and $\mathrm{H}_{2}$ density integrated over path length, $\eta=\int \zeta_{\mathrm{CR}}^{\mathrm{H}_{2}} n\left(\mathrm{H}_{2}\right) d \ell \geq$ $10^{9} \mathrm{~s}^{-1} \mathrm{~cm}^{-2}$. The $\mathrm{H}_{2}^{+}$lines are excited directly by the ionization of $\mathrm{H}_{2}$, so that their fluxes are proportional to the integral above and are insensitive to conditions like density and temperature. When the CR exposure is as high as $\eta \sim 10^{9} \mathrm{~s}^{-1} \mathrm{~cm}^{-2}$, then the distortion-induced rotational transitions of $\mathrm{H}_{3}^{+}$in the far-infrared (Black 1998, Pan \& Oka 1986) might also become detectable.

\footnotetext{
${ }^{7}$ The other TeV sources detected near W28, H.E.S.S. J1800-240 A and B, correspond to more distant star-forming regions embedded in giant molecular complexes at $3 \mathrm{kpc}$ (Sato et al. 2014). The relative angular sizes of W28 and of these massive complexes, and the linear sizes of tens of parsecs of the latter, indicate that the SNR lies too far in front of these complexes to use them as useful probes for CR diffusion.
} 


\subsection{Interstellar ion chemistry driven by cosmic rays}

Low-energy CRs, $<100 \mathrm{MeV} /$ nucleon, ionize $\mathrm{H}, \mathrm{H}_{2}$, and $\mathrm{He}$, which are otherwise well shielded from ionizing starlight in neutral interstellar gas (see Sect. 5.1). The ionization products, $\mathrm{H}^{+}, \mathrm{H}_{2}^{+}$, and $\mathrm{He}^{+}$, react with other interstellar atoms and molecules and drive an active ion-neutral chemistry even at temperatures near absolute zero. The astrochemistry directly related to CR ionization has been reviewed recently by Indriolo \& McCall (2013). In neutral regions with a high molecular fraction, $f\left(\mathrm{H}_{2}\right)=2 n\left(\mathrm{H}_{2}\right) /\left[2 n\left(\mathrm{H}_{2}\right)+n(\mathrm{H})\right] \sim 1$, almost every ionization event forming $\mathrm{H}_{2}^{+}$is followed by the rapid reaction $\mathrm{H}_{2}^{+}+\mathrm{H}_{2} \rightarrow \mathrm{H}_{3}^{+}+\mathrm{H}$. The interstellar $\mathrm{H}_{3}^{+}$molecular ion is observable by high-resolution absorption spectroscopy toward background stars at infrared wavelengths, while the highly reactive $\mathrm{H}_{2}^{+}$has not yet been observed in space.

Production of the hydrogen ions also initiates the ion chemistry of oxygen, the third most abundant element in space. Aside from the accidentally near-resonant charge transfer with protons $\mathrm{H}^{+}+\mathrm{O} \rightleftharpoons \mathrm{H}+\mathrm{O}^{+}$which is sensitive to temperature at $T<300 \mathrm{~K}$, this ion-neutral chemistry remains rapid even at the lowest interstellar temperatures. The ion chemistry of oxygen-bearing ions is thought to be well understood and supported by accurate reaction rate coefficients (Hollenbach et al. 2012). As illustrated in the reaction flow diagram (Fig. 8), the charge of CR-produced $\mathrm{H}^{+}$and $\mathrm{H}_{3}^{+}$is transferred readily to $\mathrm{OH}^{+}, \mathrm{H}_{2} \mathrm{O}^{+}$, and $\mathrm{H}_{3} \mathrm{O}^{+}$. The efficiency with which the oxygen ion sequence proceeds all the way to the saturated ion $\mathrm{H}_{3} \mathrm{O}^{+}$is limited mainly by the molecule fraction of the gas: if $f\left(\mathrm{H}_{2}\right)<<1$, then the abundance of $\mathrm{OH}^{+}$is favored. Only when $f\left(\mathrm{H}_{2}\right) \sim 1$ does formation of the highly reactive ions $\mathrm{OH}^{+}$and $\mathrm{H}_{2} \mathrm{O}^{+}$lead to $\mathrm{H}_{3} \mathrm{O}^{+}$with high efficiency. Thus the $\mathrm{H}_{2} \mathrm{O}^{+} / \mathrm{OH}^{+}$ratio is sensitive to $f\left(\mathrm{H}_{2}\right)$. In molecular regions, these ions spawn important neutral molecules $\mathrm{OH}$ and $\mathrm{H}_{2} \mathrm{O}$ in abundances that depend on the abundance of free electrons. Atomic carbon has an ionization potential less than that of atomic hydrogen and is therefore susceptible to photoionization by ultraviolet photons of the background starlight, which penetrate through diffuse molecule gas (visual extinction $A_{V} \leq 1$ magnitude). Thus carbon ion chemistry in diffuse and translucent molecular gas tends to be dominated by photoionization rather than $\mathrm{CR}$ ionization. In diffuse and translucent gas (PDR), starlight photoionization leads to $\mathrm{C}^{+}, \mathrm{CH}_{2}^{+}, \mathrm{CH}_{3}^{+}$, etc., which react with products of $\mathrm{CR}$ ionization $\left(\mathrm{OH}, \mathrm{H}_{2} \mathrm{O}\right)$ to provide the main sources of $\mathrm{HCO}^{+}$and $\mathrm{CO}$. In contrast, in dense, dark regions where $f\left(\mathrm{H}_{2}\right) \sim 1$, the reaction $\mathrm{H}_{3}^{+}+\mathrm{CO} \rightarrow \mathrm{HCO}^{+}+\mathrm{H}_{2}$ may be both the main source of $\mathrm{HCO}^{+}$and one of the main sinks of $\mathrm{H}_{3}^{+}$. In general, the abundant $\left(\mathrm{HCO}^{+} / \mathrm{H}_{2} \sim 10^{-9}\right)$ and widely observed ion $\mathrm{HCO}^{+}$is not necessarily a direct tracer of $\mathrm{CR}$ ionization: (a) its rotational emission lines readily become optically thick (saturated) so that there is not a linear relation between line intensity and column density (abundance), and (b) its formation and destruction are partly controlled by ionization processes that do not involve $\mathrm{CR}$.

The abundant $\mathrm{C}^{+}$and $\mathrm{S}^{+}$ions from photoionization in diffuse molecular gas also participate in the formation of $\mathrm{CH}^{+}$and $\mathrm{SH}^{+}$in turbulent dissipation regions (TDR), where a high-temperature chemistry is thought to operate over relatively small scales of length $\left(10^{11}-10^{13} \mathrm{~cm}\right)$ and time $\left(1-10^{2}\right.$ years $)$. Models of TDR chemistry have developed considerably in recent years (Godard, Falgarone \& Pineau Des Forêts 2009; Godard, Falgarone \& Pineau des Forêts 2014), particularly in response to Herschel observations of $\mathrm{CH}^{+}$and $\mathrm{SH}^{+}$absorption lines 
over long path lengths through the Galactic ISM (Falgarone et al. 2010a,b, Godard et al. 2012). In a very broad sense, the TDR chemistry may be related to CRs through their coupling to interstellar turbulence on large scales. There are tantalizing hints of a more active TDR chemistry in the nearby starburst galaxy $\mathrm{M} 82$, where the abundance ratio $\mathrm{CH}^{+} / \mathrm{CH}$ is much higher than in our Galaxy, which might signify a larger input of CR-driven turbulence in that galaxy (Welty et al. 2014).

The discovery of a noble-gas molecule, $\mathrm{ArH}^{+}$, together with $\mathrm{OH}^{+}$, in the Crab Nebula supernova remnant (Barlow et al. 2013) reveals an environment where molecular ions can be used to probe the competition between energetic particles and non-thermal UV/X-radiation. $\mathrm{ArH}^{+}$was subsequently shown to be widespread in interstellar absorption, where it is likely formed by the reaction $\mathrm{Ar}^{+}+\mathrm{H}_{2} \rightarrow \mathrm{ArH}^{+}+\mathrm{H}$ following CR ionization of neutral Ar (Schilke et al. 2014). Because $\mathrm{ArH}^{+}$is destroyed readily by reaction with $\mathrm{H}_{2}$, it evidently traces neutral gas of low molecular fraction, $f\left(\mathrm{H}_{2}\right) \approx 10^{-4}$ to $10^{-3}$. The column densities of $\mathrm{ArH}^{+}, \mathrm{OH}^{+}, \mathrm{H}_{2} \mathrm{O}^{+}$, and $\mathrm{HF}$, taken together, should offer a good diagnostic of the distribution of the fractional abundance of $\mathrm{H}_{2}$ (Schilke et al. 2014). Unfortunately, sensitive observations of most of these species require high-resolution spectra at submm wavelengths, best obtained with an orbiting telescope like Herschel, which completed its mission in 2013 with no successor in sight.

\subsection{Observations of molecular ions: $\mathrm{H}_{3}^{+}$}

The central role of the $\mathrm{H}_{3}^{+}$ion, produced by $\mathrm{CR}$ ionization, in interstellar chemistry (Herbst \& Klemperer 1973) was confirmed by its discovery in dense molecular clouds through high-resolution infrared spectroscopy (Geballe \& Oka 1996). The identification of $\mathrm{H}_{3}^{+}$in diffuse molecular gas (Geballe et al. 1999, Indriolo et al. 2007, Indriolo \& McCall 2012, McCall et al. 1998, 2002, 2003) as well as in dense clouds (Brittain et al. 2004, Gibb et al. 2010, Kulesa 2002, McCall et al. 1999) meant that the cosmic ray ionization rate could be inferred in two distinct chemical regimes. In the diffuse gas where the fractional ionization is high, $x(e)=n(e) / n\left(\mathrm{H}_{2}\right) \geq 10^{-4}$, and photon-dominated, the $\mathrm{H}_{3}^{+}$abundance is limited by dissociative recombination with $e^{-}$. In dense clouds that are opaque to UV radiation, $\mathrm{H}_{3}^{+}$is removed mainly by reactions with $\mathrm{O}, \mathrm{CO}$, and other neutral species.

Detections of $\mathrm{H}_{3}^{+}$in 21 out of 50 observed diffuse lines of sight were collected by Indriolo \& McCall (2012), who deduced values of the CR ionization rate of $\mathrm{H}_{2}$ in the range $(1.7 \pm 1.3) \times 10^{-16}<\zeta_{\mathrm{CR}}^{\mathrm{H}_{2}}<(10.6 \pm 8.2) \times 10^{-16} \mathrm{~s}^{-1}$ and found some upper limits as low as $\zeta_{\mathrm{CR}}^{\mathrm{H}_{2}}<0.4 \times 10^{-16} \mathrm{~s}^{-1}$. The mean value derived from the distribution of measured rates is $\left\langle\zeta_{\mathrm{CR}}^{\mathrm{H}_{2}}\right\rangle=3.5_{-3.0}^{+5.3} \times 10^{-16} \mathrm{~s}^{-1}$. These results show that the $\mathrm{CR}$ ionization rate does vary from place to place, and they confirm that the ionization rate in thicker, denser clouds tends to be lower than in the diffuse clouds. Indriolo et al. (2012) combined infrared observations of $\mathrm{H}_{3}^{+}$ with submm-wave absorption observations of $\mathrm{OH}^{+}$and $\mathrm{H}_{2} \mathrm{O}^{+}$in the same diffuse cloud toward W 51 in order to constrain the molecular hydrogen fraction in the photon-dominated part of the cloud and to deduce $\zeta_{\mathrm{CR}}^{\mathrm{H}_{2}}=(4.8 \pm 3.4) \times 10^{-16} \mathrm{~s}^{-1}$.

Crabtree et al. (2011) compared the abundances of the para and ortho nuclearspin-symmetry species of $\mathrm{H}_{2}$ and $\mathrm{H}_{3}^{+}$in diffuse molecular clouds and found that the spin temperature is lower in $\mathrm{H}_{3}^{+}$than in its neutral parent $\mathrm{H}_{2}$. A full interpretation will require better knowledge of the state-specific rates of dissociative 
recombination with electrons and might have consequences for the inferred $\mathrm{CR}$ ionization rates (Albertsson et al. 2014).

The central part of our Galaxy is clearly special. At the infrared wavelengths of the $\mathrm{H}_{3}^{+}$absorption lines, the extinction by interstellar dust is small enough that background stars in the Galactic Center can be observed with good sensitivity and high spectroscopic resolution. Thus it has been possible to map $\mathrm{H}_{3}^{+}$ across the 200 pc diameter Central Molecular Zone (CMZ) of the Galaxy (Goto et al. 2014, 2002, 2011, 2008, Oka et al. 2005), with results reviewed by Geballe (2012) and Goto et al. (2013). Although the large molecular mass of the CMZ is concentrated in dense molecular clouds, these occupy only a small fraction of its volume. The $\mathrm{H}_{3}^{+}$observations revealed large column densities and surprisingly high abundances in excited rotational states (Goto et al. 2011, Oka et al. 2005) at radial velocities distinct from those of the dominant giant molecular clouds; this implies the existence of an interesting component of dilute, $n\left(\mathrm{H}_{2}\right) \leq 100 \mathrm{~cm}^{-3}$, and warm, $T \sim 200 \mathrm{~K}$, molecular gas, with a large filling factor relative to the denser clouds (Oka \& Epp 2004). Moreover, the ionization rate in the CMZ is estimated to be $\zeta_{\mathrm{CR}}^{\mathrm{H}_{2}} \approx(2-7) \times 10^{-15} \mathrm{~s}^{-1}$ (Goto et al. 2013, Oka et al. 2005), that is, $\approx 10$ times higher than in the Galactic disk. Observations toward two stars in the central star cluster, GCIRS $1 \mathrm{~W}$ and GCIRS 3, have revealed $\mathrm{H}_{3}^{+}$ in several different kinematical components within the CMZ (Goto et al. 2014). The molecular gas at velocities +20 to $+80 \mathrm{~km} \mathrm{~s}^{-1}$ is thought to be confined very close to the GC and appears to be even hotter, $T \approx 300 \pm 50 \mathrm{~K}$, and much denser, $n\left(\mathrm{H}_{2}\right) \geq 10^{4} \mathrm{~cm}^{-3}$, than the dilute gas of the CMZ, but with a similar $\mathrm{CR}$ ionization rate, $\zeta_{\mathrm{CR}}^{\mathrm{H}_{2}}>1.6 \times 10^{-15} \mathrm{~s}^{-1}$ (Goto et al. 2014). That the ionization rate is higher than in the diffuse molecular gas of the Galactic disk is perhaps not surprising, considering that the frequency and density of supernovae must be higher in the GC. Several recent studies have assembled more comprehensive descriptions of the energetic particles and photons in the Galactic Center (Yoast-Hull, Gallagher \& Zweibel 2014; Yusef-Zadeh et al. 2013, e.g.).

\subsection{Observations of molecular ions: $\mathrm{OH}^{+}, \mathrm{H}_{2} \mathrm{O}^{+}, \mathrm{H}_{3} \mathrm{O}^{+}$}

Recent advances in submm-wave astronomy have made it possible to observe the reactive molecular ions $\mathrm{OH}_{n}^{+}(n=1$ to 3$)$ and thus to test longstanding predictions of interstellar chemical models (see Sect. 6.2). Interstellar $\mathrm{OH}^{+}$was first detected by Wyrowski et al. (2010) with the Atacama Pathfinder EXperiment (APEX) telescope shortly before the first surveys of this ion with the HIFI instrument aboard Herschel Space Observatory were completed (Gerin et al. 2010, Neufeld et al. 2010). The $\mathrm{OH}^{+}$ion can now be observed via ground-based optical spectroscopy, too (Krełowski, Beletsky \& Galazutdinov 2010; Porras et al. 2014). The water ion $\mathrm{H}_{2} \mathrm{O}^{+}$was also surveyed with Herschel absorption observations (Schilke et al. 2010, Wyrowski et al. 2010).

The abundances of the $\mathrm{OH}_{n}^{+}$ions in relation to the $\mathrm{CR}$ ionization rate have been explored through detailed models (Hollenbach et al. 2012), which complement the analytical approximations that apply in the limit of low $f\left(\mathrm{H}_{2}\right)$. The three $\mathrm{OH}_{n}^{+}$ions are also observed in absorption and/or emission in star-forming regions (Benz et al. 2013, 2010, Gupta et al. 2010, van der Tak et al. 2013), in planetary nebulae (Aleman et al. 2014, Etxaluze et al. 2014), and in the Crab Nebula (Barlow et al. 2013), but these are instances of UV/X-ray photochemistry rather than a manifestation of CRs. 
Indriolo et al. (2014) have assembled the Herschel absorption observations of the $\mathrm{OH}_{n}^{+}$ions in multiple velocity components along 20 lines of sight in the Galaxy. These observations trace diffuse gas of low molecular fraction that has a Gaussian distribution with mean and standard deviation $f\left(\mathrm{H}_{2}\right)=0.042 \pm 0.018$. The derived CR ionization rates in this component of gas outside the CMZ display a log-normal distribution with a mean $\zeta_{\mathrm{CR}}^{\mathrm{H}}=1.8 \times 10^{-16} \mathrm{~s}^{-1}$ and a standard deviation in the logarithm of 0.29. Diffuse gas near the Galactic Center is exposed to ionization rates $\sim 10^{2}$ times higher. Since $\zeta_{\mathrm{CR}}^{\mathrm{H}} \approx 0.65 \zeta_{\mathrm{CR}}^{\mathrm{H}_{2}}$, these results are completely consistent with the ionization rates derived from $\mathrm{H}_{3}^{+}$observations of diffuse molecular gas (Sect. 6.3). The ubiquity of $\mathrm{OH}^{+}, \mathrm{H}_{2} \mathrm{O}^{+}$, and $\mathrm{ArH}^{+}$(Schilke et al. 2014) in regions of low $f\left(\mathrm{H}_{2}\right)$ provides independent evidence of a significant component of neutral, molecule-containing gas that will not be traced in surveys of molecular emission lines like CO (cf. Sect. 7.1).

Unlike its highly reactive precursors $\left(\mathrm{OH}^{+}\right.$and $\left.\mathrm{H}_{2} \mathrm{O}^{+}\right)$, the $\mathrm{H}_{3} \mathrm{O}^{+}$ion is found mainly in regions of higher density and higher molecular fraction; nevertheless, it is still widespread. Herschel observations of $\mathrm{H}_{3} \mathrm{O}^{+}$line absorption over long sightlines toward Sgr B2 and W 31C revealed surprisingly large amounts of the ion in highly excited rotational energy levels $\left(E_{\text {rot }} / k \sim 1000 \mathrm{~K}\right.$ above the ground state). These rotationally "hot" molecules do not require molecular gas at high kinetic temperature $(\geq 400 \mathrm{~K})$ because the exoergic formation process $\mathrm{H}_{2} \mathrm{O}^{+}+$ $\mathrm{H}_{2} \rightarrow \mathrm{H}_{3} \mathrm{O}^{+}+\mathrm{H}$ converts the chemical energy difference between reactants and products into internal (rotational) energy of $\mathrm{H}_{3} \mathrm{O}^{+}$(Lis et al. 2014). In this interpretation, the observations place rather direct constraints on the chemical source rate of $\mathrm{H}_{3} \mathrm{O}^{+}$, and they imply $\mathrm{CR}$ ionization rates that are in harmony with the rates deduced from $\mathrm{OH}^{+}, \mathrm{H}_{2} \mathrm{O}^{+}$, and $\mathrm{H}_{3}^{+}$both in the Galactic disk and in the CMZ.

\section{Cosmic-ray tracers of the ISM}

\subsection{Tracing gas and dust properties}

Accurately tracing the gas mass at all cloud scales plays a pivotal role in understanding galaxy evolution and the gas structure that leads to star formation with a given efficiency and initial mass function. Close-up studies offer key data to test the reliability of $N_{\mathrm{H}}$ measures, commonly evaluated from Hi lines and from CO rotational emission lines as a proxy for cold $\mathrm{H}_{2}$. Herschel has recently opened access to new line surveys, among which the ubiquitous [CII] line at $158 \mu \mathrm{m}$, which arises from all gas phases except the UV-shielded $\mathrm{H}_{2}$ (Pineda et al. 2013). Thermal emission from dust grains and $\gamma$ rays from $\mathrm{CR}$ interactions with the gas nucleons complement the line data with the advantage of tracing the whole gas indifferently, but with the disadvantage of integrating $N_{\mathrm{H}}$ column densities across the whole Galaxy.

The empirical $X_{\mathrm{CO}}$ factor that relates $N_{\mathrm{H}_{2}}$ column densities and integrated ${ }^{12} \mathrm{CO}(1 \rightarrow 0)$ line intensities $\left(W_{\mathrm{CO}}\right)$ has recently been reviewed by Bolatto, Wolfire \& Leroy (2013). Across galaxies, $X_{\mathrm{CO}}$ varies only moderately with metallicity and UV-flux gradients. Past the central few kpc, it averages out to uniform values to large radii (Glover \& Mac Low 2011, Sandstrom et al. 2013). Within individual clouds, $X_{\mathrm{CO}}$ is expected to decrease from the molecular outskirts, where CO is exposed to photodissociation and weakly excited, to the dust-shielded cores where $\mathrm{CO}$ self shields and most of the carbon is locked in $\mathrm{CO}$ instead of $\mathrm{C}^{+}$, but where 
${ }^{12} \mathrm{CO}$ lines become saturated. Large $X_{\mathrm{CO}}$ fluctuations may result from local imbalance between CO formation and dissociation in the optically thin envelopes (Glover \& Clark 2012, Lee et al. 2014, Sheffer et al. 2008), but they may average out along sightlines (Liszt, Pety \& Lucas 2010).

The $\mathrm{HI}-\mathrm{H}_{2}$ interface is rich in dense and opaque $\mathrm{HI}$, and in CO-faint or COdark $\mathrm{H}_{2}$ (van Dishoeck \& Black 1988; Visser, van Dishoeck \& Black 2009; Wolfire, Hollenbach \& McKee 2010). Both the atomic and molecular parts of this interface can easily escape Hi and CO surveys. Early dust studies hinted at the presence of dust excesses over the $\mathrm{HI}$ and $\mathrm{CO}$ contributions (e.g., Blitz, Bazell \& Desert 1990), but the importance of this "dark neutral medium" (DNM) interface in the interstellar mass budget has been revealed by jointly using CRs and dust to trace large gas masses not accounted for in $\mathrm{HI}$ and $\mathrm{CO}$ emission (Grenier, Casandjian $\&$ Terrier 2005). The correlation between the two tracers is essential to rule out localized variations in dust-to-gas ratio, dust opacity, or CR flux (Planck et al. 2014). The first $\gamma$-ray and dust study revealed a DNM mass equal to about $30 \%$ of the CO-bright mass in the local ISM (Grenier, Casandjian \& Terrier 2005). Later studies have increased this local fraction to $118 \%$ and $164 \%$, but with the sole use of dust to estimate the mass (Paradis et al. 2012, Planck Collaboration et al. 2011b). Dust and [CII] line analyses suggest that the DNM fractional mass increases with radius in the Galaxy (Langer et al. 2014, Pineda et al. 2013, Planck Collaboration et al. 2011a). In the absence of extensive [CII], $\mathrm{CH}$ or $\mathrm{OH}$ surveys, and given the difficult [CII] separation between the DNM and the other neutral and ionized phases (Langer et al. 2014), one must presently rely on the spatial correlation between $\gamma$-ray and dust excesses over $\mathrm{HI}$ and $\mathrm{CO}$ expectations in a cloud to quantify and map the elusive DNM phase. We look forward to the first confrontations of the DNM maps with [CI], [CII], $\mathrm{CH}$, and $\mathrm{OH}$ line data in nearby clouds, away from the Galactic plane confusion, to learn more about the DNM chemical composition.

CRs expose the total gas to view, regardless of its thermodynamical and chemical state, thereby enabling the calibration of other tracers as well as measurements of $\mathrm{HI}$ opacities and $X_{\mathrm{CO}}$ ratios. The $N_{\mathrm{H}}$ tracing relies on the assumption of a uniform $\mathrm{CR}$ flux through the $\mathrm{HI}$ and $\mathrm{H}_{2}$ phases of a given cloud. The large $\mathrm{CR}$ diffusion lengths in the ISM and the observed uniformity of CR spectra across gas phases give weight to this assumption (see Sect. 3.1.1). CRs have thus long been used to unveil molecular clouds (e.g., Black \& Fazio 1973, Lebrun \& Paul 1983), to calibrate $X_{\mathrm{CO}}$ (Strong et al. 1988, and references therein), and to discover DNM envelopes of nearby clouds (Grenier, Casandjian \& Terrier 2005). Such studies have leaped forward with Fermi LAT observations. We briefly summarize results obtained so far in the different regions referred to in Fig. 4.

7.1.1 IN THE Hi: The choice of Hi spin temperature modifies the contrast between peaks and troughs in the derived $N_{\mathrm{HI}}$ maps. This choice can be tested against the $\gamma$-ray intensity produced in the Hi gas (Abdo et al. 2010g, Ackermann et al. 2012f, Planck et al. 2014). The results obtained for the Cepheus and Cygnus clouds agree with the precise, but sparse, values measured with paired absorption/emission HI spectra, but such tests are restricted to uniform spin temperatures so far.

7.1.2 IN THE DNM: DNM column-density maps in nearby clouds show that this phase occupies a volume and exhibits column densities close to that of the dense Hi around the CO-bright cores (Abdo et al. 2010g, Ackermann 
et al. 2012d,f, Planck et al. 2014). Comparing the mass locked in the Hi-bright, DNM, and CO-bright phases in the current sample of $10^{4}-10^{6} \mathrm{M}_{\odot}$ clouds, we find that the DNM mass compares with the CO-bright one (40-190\%), but that it represents only about a fifth of the Hi-bright mass. Compared to the total mass in a cloud, the DNM often amounts to less than $20 \%$ because of the predominant $\mathrm{HI}$ gas, but there can be large deviations: two nearby complexes in the $2005 C G R O$ EGRET sample reached mass fractions of $35 \%$ and $60 \%$. The low fractions measured locally compare with the average near $20 \%$ found with [CII] in the Galactic disc for clouds exhibiting dense CO (Langer et al. 2014). We caution, however, that mass fractions involving the Hi mass can be uncertain because of the ill defined outer Hi edge of a cloud in the observations.

Dust UV shielding in the DNM phase is essential to theoretically explain the HI- $\mathrm{H}_{2}$ transition and the CO observations (Lee et al. 2014): the larger the visual extinction, $A_{V}$, the thinner the CO photodissociation layer (see Fig. 17 of Langer et al. 2014 and Wolfire, Hollenbach \& McKee 2010). Figure 9 shows $\gamma$-ray estimates of the $A_{V} / N_{\mathrm{H}}$ ratios in the DNM, with values comparable to those found in the HI gas. Yet, we observe a puzzling evolution of the DNM fractional mass with $A_{V}$ in separate clouds of the same complex (Planck et al. 2014): its rise with the average $A_{V}$ across the CO-bright part is at odds with the marked decline expected from the thinning of the $\mathrm{CO}$ photodissociation layer. Models also predict that $\sim 30 \%$ of the $\mathrm{H}_{2}$ mass should be CO-dark (Levrier et al. 2012; Wolfire, Hollenbach \& McKee 2010). The CO-dark $\mathrm{H}_{2}$ fractions shown in Fig. 10 often exceed the theoretical expectations whether we adopt a half or fully molecular composition for the DNM. In view of these results, we cannot conclude whether the DNM is mostly atomic or molecular, especially since the $\mathrm{HI} \leftrightarrow \mathrm{H}_{2}$ transition is very dynamical in space and time (Glover \& Mac Low 2011). This uncertainty justifies the neutral DNM appellation over the "CO-dark $\mathrm{H}_{2}$ " one also in use.

7.1.3 About $X_{\mathrm{CO}}$ : Recent $X_{\mathrm{CO}}$ measurements made in $\gamma$ rays in the local ISM and in the outer Galaxy are shown in Figure 9, together with the $X_{\mathrm{CO}}$ profile obtained from the radiative transfer of ${ }^{12} \mathrm{CO}$ and ${ }^{13} \mathrm{CO}$ lines, assuming variations in $[\mathrm{CO}] /\left[\mathrm{H}_{2}\right]$ abundance linked to the metallicity gradient (Pineda et al. 2013). The $\gamma$-ray estimates require no assumption about a metallicity or CR-density gradient, only that of a uniform CR penetration between the HI and CO-bright gas of a given complex, kinematically separated from other clouds along sightlines. Both profiles may jointly hint at an $X_{\mathrm{CO}}$ increase with Galactic radius. It would be more pronounced than in external galaxies, but caution is strongly advised since the CO-transfer estimates reflect the input metallicity gradient, and the $\gamma$-ray signal from the outer arm is faint and point-source contamination difficult to remove.

The $\gamma$-ray data yield an average $X_{\mathrm{CO}}$ value at parsec scales in the Gould Belt clouds of $0.9 \times 10^{20} \mathrm{~cm}^{-2} \mathrm{~K}^{-1} \mathrm{~km}^{-1} \mathrm{~s}$ and a kpc-scale average in the Local and Perseus Arms of $1.9 \times 10^{20} \mathrm{~cm}^{-2} \mathrm{~K}^{-1} \mathrm{~km}^{-1} \mathrm{~s}$. The low $X_{\mathrm{CO}}$ values in nearby clouds compare well with high-resolution estimates based on $\mathrm{CH}$ data or molecular chemistry in diffuse clouds (Cotten \& Magnani 2013; Liszt, Pety \& Lucas 2010). The local-arms average is only $30 \%$ larger than the mean found in external galaxies (Sandstrom et al. 2013). The factor of 2 difference between the Gould Belt and local arms cannot be attributed to metallicity variations. Diffuse molecular envelopes cover larger surfaces than dense cores in well-resolved nearby clouds. This would bias the cloud average $X_{\mathrm{CO}}$ upward, not downward, as $X_{\mathrm{CO}}$ 
increases toward cloud edges (Cotten \& Magnani 2013; Lee et al. 2014; Liszt, Pety \& Lucas 2010). It remains to be checked whether the difference stems from a bias in the component separation between the CO-bright and much more massive HI and DNM phases. It can result from pile-up along sightlines and/or the increased level of cross-talk in the radio maps of distant complexes. Observations of face-on versus edge-on external galaxies do indicate a larger $X_{\mathrm{CO}}$ ratio in the latter (Sandstrom et al. 2013).

7.1.4 ON DUST PROPERTIES: CRs have recently been used to quantify how dust emission properties, per gas nucleon, vary from the diffuse $\mathrm{HI}$ to the dense $\mathrm{H}_{2}$ environments in the translucent Chamaeleon clouds (Planck et al. 2014). They have revealed a 2- to 4.6-fold rise in grain emission opacity as the gas becomes denser and grains less heated, as attested by a 2-fold drop in their radiated power. Figure 11 shows these important trends. They confirm the opacity changes recently noted inside the Hi phase (Planck Collaboration et al. 2014b), or from comparisons between dust emission and absorption data (Martin et al. 2012, Roy et al. 2013), with the CR advantage of independently accounting for the total $N_{\mathrm{H}}$ in the opaque $\mathrm{HI}, \mathrm{DNM}$, and CO-bright $\mathrm{H}_{2}$ gas. The opacity variations presumably indicate a chemical and/or structural change of the grains, perhaps in response to ice-coating and aggregation. The magnitude of the variations can severely bias the derivation of $X_{\mathrm{CO}}$ to high values when using dust emission to trace the gas (e.g. by $190 \%$ in the Chamaeleon complex, see Figure 9). The magnitude of the opacity variations also raises a cautionary flag for the use of dust emission to retrieve Hi optical depths and spin temperatures in the opaque CNM (Fukui et al. 2014).

Further comparisons between $\gamma$-ray and dust-absorption determinations of $X_{\mathrm{CO}}$ are of crucial importance to assess whether the cloud-to-cloud variations found in the literature stem from the choice of total gas tracer, from the sampling scale in the cloud, or from environmental differences. The same remark holds for cloudto-cloud variations in dust emission or absorption opacities which can be gauged with the independent $\gamma$-ray data. The clouds off the Galactic plane exhibit large differences in emission opacity (Planck Collaboration et al. 2014a), which may largely be due to the DNM column densities missing in the gas census in this work (compare these variations with the sky maps of Planck Collaboration et al. 2011b and Grenier, Casandjian \& Terrier 2005). Yet, accounting for all forms of $\gamma$-ray emitting gas in the Chamaeleon clouds leaves unexplained 2-fold differences in emission opacity and specific power between these clouds and the all-sky average (Planck et al. 2014): they may hint at cloud-to-cloud, therefore environmental changes in dust properties. The new $\mathrm{mm}$ and $\gamma$-ray all-sky surveys open the way to stimulating studies to explore the origin of grain evolution in a variety of environments.

\subsection{Tracing cosmic-ray leptons and the magnetic field}

Synchrotron emission from CR electrons and positrons give an essential complementary view (to $\gamma$ rays) of the distribution and spectrum of leptons throughout the Galaxy. Since synchrotron also depends on the magnetic field, the joint analysis with $\gamma$ rays should allow an unambiguous picture to be developed. While this has not been completed yet, comparison of CR propagation models with synchrotron data (Jaffe et al. 2011; Lineros, Bringmann \& Donato 2012; Strong, Orlando \& Jaffe 2011), shows the power of this approach. It illustrates the use 
of low-frequency synchrotron (below a few $100 \mathrm{MHz}$ ) to probe leptons in the regions where solar modulation makes direct measurements problematic, and at the same time providing an interstellar spectrum for testing modulation models themselves. The comparison with $\mathrm{CR}$ propagation models also emphasizes the importance of secondary electrons and positrons in addition to primary CR electrons for interpreting synchrotron spectra, espacially at low frequency (see Fig 12, (Strong, Orlando \& Jaffe 2011)). Note however that these model spectra do not include the contribution from the positrons responsible for the excess above 10 GeV detected by PAMELA, Fermi-LAT and AMS-02, which are presumably primary (see Section 2.4). This will increase the intensity at frequencies above a few $\mathrm{GHz}$, and is important to consider in future.

The study of Galactic synchrotron radiation was extended to full-sky spatial distribution and polarization data by Orlando \& Strong (2013), Sun et al. (2008) using a range of magnetic field models from the literature. They provide useful constraints on the strengths of the regular and anisotropic fields in the disc and of the toroidal field in the halo. They also explore the height of the CR electron halo. The role of old supernova remnants in the form of 'radio loops' was studied by Mertsch \& Sarkar (2013). They conclude that the loops may make an important contribution to the fine structure of the synchrotron sky.

The magnetic field model of Jaffe et al. (2011), using the Hammurabi software (Waelkens et al. 2009) to compute synchrotron, includes a so-called striated field with reversals, which affect rotation measures but not the synchrotron emission. A new model by Jansson \& Farrar (2012a) based on WMAP radio surveys and 40000 extragalactic rotation measures was compared with synchrotron data in Jansson \& Farrar (2012b), and with WMAP polarization data in Farrar (2014). This model includes the $\mathrm{X}$-shape feature in the magnetic field observed in external galaxies, and unlike other previous models, the field is divergence-free - a physical constraint on possible field configurations.

Spectral index variation of polarized emission measured by WMAP (Fuskeland et al. 2014) will provide essential information both on the regular magnetic field and CR lepton propagation.

For an extensive review of the whole subject of magnetic fields in galaxies see Beck \& Wielebinski (2013) and also Haverkorn (2015). These cover also external galaxies, like the face-on grand-design spiral M51. But we caution against the extensive use in such work of the so-called equipartition method, which assumes an equality of energy in CRs and magnetic fields, and for which there is no real justification.

\section{Cosmic rays in star-burst environments}

\subsection{Effect on cosmic-ray composition}

It has long been expected that massive-star forming regions host CR factories since $\sim 80 \%$ of all Galactic supernovae are core collapse explosions with progenitors formed in OB associations (Higdon \& Lingenfelter 2005). Shockwaves of thermonuclear and core-collapse supernovae sweep material of different metallicities $\left(1.3 Z_{\odot}\right.$ in the ISM, $2-3 Z_{\odot}$ in superbubbles, Lingenfelter \& Higdon 2007), so the CR composition holds a record of the mixing of supernova ejecta, progenitor wind, and ISM gas in the acceleration site. ${ }^{22} \mathrm{Ne} /{ }^{20} \mathrm{Ne}, \mathrm{Si} / \mathrm{Fe}$, and $\mathrm{N}-$ $\mathrm{Sr}$ abundances give a record integrated over 1-20 Myr in the local spiral arms 
(Lingenfelter \& Higdon 2007). Both refractory and volatile elements indicate a mixing by mass of $80 \%$ of older ISM gas with $20 \%$ of ejecta and wind material (Rauch et al. 2009). The lack of ${ }^{59} \mathrm{Ni}$ in the CR composition also sets a minimum timescale of $\sim 0.1$ Myr between the synthesis and acceleration of heavy nuclei (Binns et al. 2007, Wiedenbeck et al. 1999). It is the time required for ${ }^{59} \mathrm{Ni}$ to decay by electron capture whereas bare nuclei, stripped of their electrons at the CR-accelerating shock, would survive. It has been argued that the average time between supernovae in an OB association is long enough compared to the ${ }^{59} \mathrm{Ni}$ half-life to prevent substantial acceleration of recently synthesized ${ }^{59} \mathrm{Ni}$ before it can decay (Binns et al. 2007). Little is known, however, on the potential acceleration of ${ }^{59} \mathrm{Ni}$ (before decay) by the powerful stellar wind shocks and, in general, on CR propagation in the highly turbulent media characterizing starburst regions, and on the collective action of multiple shockwaves to accelerate CRs or to reaccelerate older CRs passing by (Bykov \& Toptygin 2001, Ferrand \& Marcowith 2010). Parizot et al. (2004) suggest that collective effects in superbubbles are important for CR acceleration to knee energies.

Fermi LAT observations have at last opened a new window on high-energy particles in these active regions, both in the Milky Way and in external galaxies.

\subsection{A cocoon of young cosmic rays in Cygnus $\mathrm{X}$}

Bright stellar clusters in the Cygnus X region, with high densities of massive stars and very young ages of 3-6 Myr, have carved their parent clouds over tens of parsecs through the combined action of their intense ionizing radiation and energetic winds. The latter can develop a total energy comparable to that of a supernova over the few-Myr period of strong winds (Ackermann et al. 2011a, Parizot et al. 2004). The 1-100 GeV images from the Fermi-LAT have revealed an extended source of hard emission that fills the region bounded by the ionization fronts as in a cocoon (see Fig. 13). The emission spectrum may break around a few $\mathrm{TeV}$ and extend to beyond $20 \mathrm{TeV}$ if the hardly-resolved $\mathrm{TeV}$ sources observed in this direction are the counterparts to the $10 \mathrm{GeV}$ cocoon (Bartoli et al. 2014). The hard $\gamma$ rays cannot be explained by inverse Compton scattering of the large stellar and interstellar radiation fields in the region, nor by hadronic interactions of Galactic CRs in the large gas masses (see Fig. 13). The emission requires a hard distribution of probably freshly-accelerated CR nuclei, with a spectrum close to $E_{C R}^{-2.4}$ and a total energy of only $10^{42} \mathrm{~J}$ above $2 \mathrm{GeV} /$ nucleon (Ackermann et al. 2011a). Their origin is unknown. They may have been accelerated by a supernova remnant hidden in the region, or by the dispersed ensemble of stellarwind shocks and supersonic turbulence in the cocoon (Ackermann et al. 2011a, online supplement). They may also be older Galactic CRs re-energized by the windpowered magnetic turbulence. It will require detailed models of this complex, dense, and high-pressure environment to decide among these scenarios.

The cocoon detection raises another important issue on the early propagation of CRs, once they escape from their accelerator and before they travel at large in and off the Galactic disc. The conditions prevailing in the cocoon suggest a regular magnetic field strength of $2 \mathrm{nT}$ (if in pressure equilibrium with the gas) and an energy-containing scale of $10 \mathrm{pc}$ for the turbulence. With magnetic turbulence near saturation, the CR diffusion length in the cocoon can be 100 times smaller than in the normal ISM, thereby implying an efficient proton confinement over

$100 \mathrm{kyr}$, in agreement with the ${ }^{59} \mathrm{Ni}$ timescale. The question then arises of the 
actual CR spectrum leaking out of starburst bubbles and impinging the remains of the parent clouds, at $\mathrm{GeV}-\mathrm{TeV}$ energies relevant for diffusion diagnostics at larger kpc scales, and at sub-GeV energies relevant for ionizing and heating the surrounding clouds.

\subsection{Normal galaxies}

Nearby external galaxies provide the opportunity to study the CR distributions via the outside view of their radio and $\gamma$-ray emission, in order to investigate the key role played by magnetic fields and CRs in the feedback between star formation activity and the ISM dynamics. Unfortunately the $\gamma$-ray flux from normal galaxies is low and the poor angular resolution severely limits the imaging possibilities. Only M31 and the Large and Small Magellanic clouds (LMC and SMC) have been detected so far by Fermi LAT (Abdo et al. 2010a,e,f). The total emission from M31 is consistent with a CR density similar to that in our Galaxy, whereas the lower values found in the LMC and SMC $(<30 \%$ and $<15 \%$ of the Galactic value) confirm the production within galaxies of $\mathrm{GeV}-\mathrm{TeV}$ CRs, as first evidenced by the CGRO-EGRET observations (Sreekumar et al. 1993). The LMC emission is interestingly concentrated in the 30 Doradus starburst region, and it does not correlate well with the gas in the early maps recorded by Fermi LAT even though CRs can sample the mean ISM density in a starburst environment over a large range of interstellar conditions (Boettcher et al. 2013). Spatial correlations between the warm dust emission, radio synchrotron emission, and $\mathrm{GeV} \gamma$ rays indicate a 50 times shorter diffusion length for $20-\mathrm{GeV}$ CR protons in the vicinity of 30 Doradus than in the Galactic ISM (Murphy et al. 2012). This low value compares favourably with the expectation quoted above for the turbulent medium of Cygnus X.

Radio interferometric observations of external galaxies enable studies of CR electrons and magnetic fields. Face-on spirals like M51 can be studied with resolutions down to about $200 \mathrm{pc}$ and reveal the relation of CR electrons to spiral arms and to the compression of the regular and turbulent parts of the magnetic field (Fletcher et al. 2011). Galaxies have synchrotron halos extending to several kpc from the disk, and observations of those seen edge-on allow a study of the vertical propagation of $\mathrm{CR}$ electrons and magnetic fields in their halos. A large project (CHANG-ES) to observe 35 edge-on galaxies with the JVLA is in progress and will provide a valuable resource for such work (Irwin et al. 2012a,b, 2013). Most observations are taken at $\mathrm{GHz}$ frequencies to obtain the angular resolution required, but lower frequencies are more suited for synchrotron studies by reducing the contribution from the free-free emission. Extension to $100 \mathrm{MHz}$ with the LOFAR array is now starting (Mulcahy et al. 2014) and will be followed by the Square Kilometer Array (SKA) ${ }^{8}$ down to about $300 \mathrm{MHz}$.

\subsection{Starburst galaxies}

The discovery of a correlation between the far-infrared and radio luminosities of galaxies (Helou, Soifer \& Rowan-Robinson 1985) has highlighted the close relationship between the CR electron energy content of a galaxy and its star formation rate (SFR). Such a correlation can be explained via the supernova rate if the galaxy is a good electron calorimeter, in other words if they lose

\footnotetext{
${ }^{8}$ https://www.skatelescope.org/
} 
their energy to synchrotron radiation inside the galaxy (Voelk 1989). A similar correlation would hold between the FIR and $\gamma$-ray luminosity if the galaxy is a good proton calorimeter. Reality is complicated by variations in magnetic field strengths which impact synchrotron losses, by non-radiative losses such as ionization in dense clouds, by secondary lepton production in dense clouds, and advection of CRs and magnetic fields in a galactic wind (e.g. Boettcher et al. 2013, Yoast-Hull et al. 2013a). Starburst galaxies provide extreme test cases of these scaling relations. Four objects (M82, NGC 253, NGC 1068, and NGC 4945) have been detected by Fermi LAT with $\gamma$-ray luminosities that scale with the SFR measured via FIR or radio-continuum luminosities (Abdo et al. 2010c, Ackermann et al. 2012e). M 82and NGC 253 have also been detected at higher energies with VERITAS and HESS, respectively (Acero et al. 2009, VERITAS Collaboration et al. 2009). The level of CR versus AGN activity in other starburst detections at $\mathrm{GeV}$ and $\mathrm{TeV}$ energies is unclear, although progress is being made (Yoast-Hull et al. 2014).

The first interpretations of the scaling laws and radio $+\gamma$-ray spectra vary in their approximations, but they point to large calorimetric efficiencies for the CR electrons and to efficiencies below $50 \%$ for the protons (Abramowski et al. 2012, Ackermann et al. 2012e, Paglione \& Abrahams 2012, Yoast-Hull et al. 2013b). The hard and flat spectra recorded to $\mathrm{TeV}$ energies favour energy-independent loss mechanisms for the CR nuclei, over the diffusive losses which likely shape the $\gamma$-ray spectra observed in quiescent galaxies. Together with the low calorimetric efficiency, they suggest that CR nuclei are predominantly advected away from the starburst nucleus, taking away their energy and lepton production potential. The combined interpretation of the radio and $\gamma$-ray spectra of specific galaxies can help constrain the gas densities, magnetic field strengths, and ionization rates in these dense and crowded environments, independent of minimum-energy assumptions (Paglione \& Abrahams 2012). Detailed studies of these examples are essential to evaluate the impact of CRs on the ISM in CR-dominated regions.

Radio interferometry makes it possible to identify individual supernovae and young supernova remnants in highly obscured regions of starburst galaxies like Arp 220 (Batejat et al. 2011, Lonsdale et al. 2006, Parra et al. 2007) and M 82 (Fenech et al. 2008, Gendre et al. 2013), and to infer a SN event rate as high as $4 \pm 2 \mathrm{yr}^{-1}$ in the former. The upper limit of $<0.2 \mathrm{yr}^{-1}$ (Rampadarath et al. 2014) in the center of the weak starburst NGC 253 may constrain the interpretation of the $\gamma$-rays detected there. CR-driven ion chemistry produces reactive molecular ions such as $\mathrm{OH}^{+}, \mathrm{H}_{2} \mathrm{O}^{+}$, and $\mathrm{H}_{3} \mathrm{O}^{+}$, which can now be observed in starburst and active galaxies (Aalto et al. 2011, González-Alfonso et al. 2013, Kamenetzky et al. 2012, Pereira-Santaella et al. 2013, Rangwala et al. 2011, Spinoglio et al. 2012, van der Werf et al. 2010). The observations of molecular ions have been used to infer a high rate of ionization $\zeta_{\mathrm{CR}}^{\mathrm{H}_{2}}>10^{-13} \mathrm{~s}^{-1}$ in Arp 220 (GonzálezAlfonso et al. 2013) $\sim \sim 10^{2}$ times higher than that in the Central Molecular Zone of the Milky Way - and to trace massive galactic winds.

\section{5 $\gamma$-rays in extragalactic space}

The extragalactic $\gamma$-ray background (EGB) is a basic observable complementary to those at radio, infrared, optical, and X-ray energies. The multi-wavelength extragalactic spectrum contains important information on cosmology, intergalactic CRs, etc. The determination of the EGB is critically dependent on the mod- 
elling of the Galactic foreground, in particular from the CR halo, which is still quite uncertain. Hence there is an essential coupling between CR and EGB studies. Significant progress has been made in the determination of the EGB spectrum with Fermi-LAT (The Fermi LAT collaboration et al. 2014). Both the improvement in data and modelling of the Galactic emission has contributed to the accuracy of the EGB determination. Now we have to distinguish the real EGB, which is the true intergalactic $\gamma$-ray flux, from that due to Galactic (e.g., ms pulsars) and extragalactic sources (e.g., AGN) below the detection threshold, and to other possible diffuse processes that might be attributable to dark matter. The part of the EGB due to known sources (mostly AGN) is comparable to the undetected sources part at the present time (Abdo et al. 2010b). The latter will decrease as the observations go deeper with time. The unresolved population of normal and starburst galaxies may play an important role and dominate the EGB at few $\mathrm{GeV}$ because of their hadronic pion emission (Ackermann et al. 2012e).

\section{A few more words}

The numerous interactions of sub- $\mathrm{TeV}$ CRs with their environment constitute a lively topic part of the dynamics of the interstellar medium. The latest direct measurements performed near the Earth and heliopause have reached a level of accuracy that starts to unravel "imperfections" in CR characteristics (spectra, composition, and anisotropy) of great value for better understanding their origin and modes of propagation in different media. Multi-wavelength data, in particular $\gamma$ rays, sub-mm and low-frequency radio surveys, and emission lines from chemical compounds are rapidly expanding our capability to remotely probe CR populations across decades in energy and in a large variety of Galactic environments, from the starburst nucleus to the quiet periphery of the Milky Way, and to nearby galaxies. These are important achievements and with the preparation of new CR instruments (e.g., CALET) and telescopes (e.g., SKA, HAWC, CTA, Gamma-400, LHAASO) the prospects are bright for making further progress on the long list of open questions that sprinkle the present review. CRs are major actors in the feedback between high-energy events in galaxies and the interstellar medium, but we still know very little, both observationally and theoretically, on how this feedback operates from the microscales of plasma physics and shocks to the large scales of galactic fountains and winds. Studying these feedback processes and finding ways to probe the dark side of cosmic rays (the low-energy ionizing ones) are stimulating challenges for the coming years.

\section{Related Resources}

NASA ADS compilation of references in this review and other relevant literature:

http://adsabs.harvard.edu/cgi-bin/nph-abs_connect?library\&libname=AnnRev201

\section{Acknowledgements}

We thank the Lorentz Centre for hosting a most inspiring workshop ${ }^{9}$ on CR interactions, which laid the foundations of many discussions presented in this

\footnotetext{
${ }^{9}$ http://www.lorentzcenter.nl/lc/web/2011/435/info.php3?wsid=435\&venue=Oort
} 
review. I. G. gratefully thanks the Institut Universitaire de France for its research support.

\section{References}

1. Aalto S, Costagliola F, van der Tak F, Meijerink R. 2011. Astron. Astrophys. 527:A69

2. Abdo AA, Abeysekara U, Allen BT, Aune T, Berley D, et al. 2012. Astrophys. J. $753: 159$

3. Abdo AA, Ackermann M, Ajello M, Allafort A, Atwood WB, et al. 2010a. Astron. Astrophys. 523:L2

4. Abdo AA, Ackermann M, Ajello M, Antolini E, Baldini L, et al. 2010b. Astrophys. J. 720:435-453

5. Abdo AA, Ackermann M, Ajello M, Atwood WB, Axelsson M, et al. 2009. Astrophys. J. 703:1249-1256

6. Abdo AA, Ackermann M, Ajello M, Atwood WB, Axelsson M, et al. 2010c. Astrophys. J. 709:L152-L157

7. Abdo AA, Ackermann M, Ajello M, Atwood WB, Baldini L, et al. 2010d. Science $328: 725-$

8. Abdo AA, Ackermann M, Ajello M, Atwood WB, Baldini L, et al. 2010e. Astron. Astrophys. 512:A7

9. Abdo AA, Ackermann M, Ajello M, Baldini L, Ballet J, et al. 2010f. Astron. Astrophys. 523:A46

10. Abdo AA, Ackermann M, Ajello M, Baldini L, Ballet J, et al. 2010g. Astrophys. J. $710: 133-149$

11. Abdo AA, Ackermann M, Ajello M, Baldini L, Ballet J, et al. 2011. Astrophys. J. $734: 116$

12. Abe K, Fuke H, Haino S, Hams T, Hasegawa M, et al. 2012. Physical Review Letters 108:051102

13. Abgrall H, Roueff E, Liu X, Shemansky DE. 1997. Astrophys. J. 481:557-566

14. Abramowski A, Acero F, Aharonian F, Akhperjanian AG, Anton G, et al. 2012. Astrophys. J. 757:158

15. Acero F, Aharonian F, Akhperjanian AG, Anton G, Barres de Almeida U, et al. 2009. Science 326:1080-

16. Ackermann M, Ajello M, Albert A, Allafort A, Atwood WB, et al. 2012a. Astrophys. J. Suppl. 203:4

17. Ackermann M, Ajello M, Allafort A, Antolini E, Baldini L, et al. 2012b. Astrophys. J. $756: 4$

18. Ackermann M, Ajello M, Allafort A, Atwood WB, Baldini L, et al. 2012c. Physical Review Letters 108:011103

19. Ackermann M, Ajello M, Allafort A, Atwood WB, Baldini L, et al. 2013a. Astrophys. J. Suppl. 209:34

20. Ackermann M, Ajello M, Allafort A, Baldini L, Ballet J, et al. 2011a. Science $334: 1103-$ 
21. Ackermann M, Ajello M, Allafort A, Baldini L, Ballet J, et al. 2012d. Astrophys. J. $755: 22$

22. Ackermann M, Ajello M, Allafort A, Baldini L, Ballet J, et al. 2012e. Astrophys. J. $755: 164$

23. Ackermann M, Ajello M, Allafort A, Baldini L, Ballet J, et al. 2012f. Astron. Astrophys. 538:A71

24. Ackermann M, Ajello M, Allafort A, Baldini L, Ballet J, et al. 2013b. Science 339:807-811

25. Ackermann M, Ajello M, Atwood WB, Baldini L, Ballet J, et al. 2010. Phys. Rev. D. 82:092004

26. Ackermann M, Ajello M, Atwood WB, Baldini L, Ballet J, et al. 2012g. Astrophys. J. $750: 3$

27. Ackermann M, Ajello M, Baldini L, Ballet J, Barbiellini G, et al. 2011b. Astrophys. J. $726: 81$

28. Ackermann M, Albert A, Atwood WB, Baldini L, Ballet J, et al. 2014. Astrophys. J. 793:64

29. Adriani O, Barbarino GC, Bazilevskaya GA, Bellotti R, Bianco A, et al. 2013a. Physical Review Letters 111:081102

30. Adriani O, Barbarino GC, Bazilevskaya GA, Bellotti R, Boezio M, et al. 2009. Nature 458:607-609

31. Adriani O, Barbarino GC, Bazilevskaya GA, Bellotti R, Boezio M, et al. 2011. Science 332:69-

32. Adriani O, Barbarino GC, Bazilevskaya GA, Bellotti R, Boezio M, et al. 2013b. Astrophys. J. 770:2

33. Adriani O, Barbarino GC, Bazilevskaya GA, Bellotti R, Boezio M, et al. 2013c. Astrophys. J. 765:91

34. Adriani O, Barbarino GC, Bazilevskaya GA, Bellotti R, Boezio M, et al. 2014. Astrophys. J. 791:93

35. Adriani O, Barbarino GC, Bazilevskaya GA, Boezio M, Bogomolov EA, et al. 2013d. Advances in Space Research 51:219-226

36. Aguilar A, Ajello JM, Mangina RS, James GK, Abgrall H, Roueff E. 2008. Astrophys. J. Suppl. 177:388-407

37. Aguilar M, Alberti G, Alpat B, Alvino A, Ambrosi G, et al. 2013. Physical Review Letters 110:141102

38. Aguilar M, Alcaraz J, Allaby J, Alpat B, Ambrosi G, et al. 2011. Astrophys. J. $736: 105$

39. Aharonian F, Akhperjanian AG, Anton G, Barres de Almeida U, Bazer-Bachi AR, et al. 2009. Astron. Astrophys. 508:561-564

40. Aharonian F, Akhperjanian AG, Barres de Almeida U, Bazer-Bachi AR, Becherini Y, et al. 2008. Physical Review Letters 101:261104

41. Aharonian F, Akhperjanian AG, Bazer-Bachi AR, Beilicke M, Benbow W, et al. 2006. Nature 439:695-698

42. Ahn HS, Allison P, Bagliesi MG, Barbier L, Beatty JJ, et al. 2009. Astrophys. J. 707:593-603 
43. Ahn HS, Allison PS, Bagliesi MG, Barbier L, Beatty JJ, et al. 2010. Astrophys. J. 715:1400-1407

44. Ahn HS, Allison PS, Bagliesi MG, Beatty JJ, Bigongiari G, et al. 2008. Astroparticle Physics 30:133-141

45. Albertsson T, Indriolo N, Kreckel H, Semenov D, Crabtree KN, Henning T. 2014. Astrophys. J. 787:44

46. Aleman I, Ueta T, Ladjal D, Exter KM, Kastner JH, et al. 2014. Astron. Astrophys. 566:A79

47. Aloisio R, Blasi P. 2013. JCAP 7:1

48. Amato E. 2014. International Journal of Modern Physics D 23:30013

49. Barkov MV, Bosch-Ramon V. 2014. Astron. Astrophys. 565:A65

50. Barlow MJ, Swinyard BM, Owen PJ, Cernicharo J, Gomez HL, et al. 2013. Science 342:1343-1345

51. Bartoli B, Bernardini P, Bi XJ, Branchini P, Budano A, et al. 2014. Astrophys. J. $790: 152$

52. Batejat F, Conway JE, Hurley R, Parra R, Diamond PJ, et al. 2011. Astrophys. J. $740: 95$

53. Battiston R. 2014. Physics of the Dark Universe 4:6-9

54. Beck R. 2012. Space Sci. Rev. 166:215-230

55. Beck R, Wielebinski R. 2013. Magnetic Fields in Galaxies. 641

56. Becker JK, Black JH, Safarzadeh M, Schuppan F. 2011. Astrophys. J. 739:L43

57. Benz AO, Bruderer S, van Dishoeck EF, Stäuber P, Wampfler SF. 2013. Journal of Physical Chemistry A 117:9840-9847

58. Benz AO, Bruderer S, van Dishoeck EF, Stäuber P, Wampfler SF, et al. 2010. Astron. Astrophys. 521:L35

59. Berezhko EG, Ksenofontov LT. 2014. Astrophys. J. 791:L22

60. Berezhko EG, Ksenofontov LT, Ptuskin VS, Zirakashvili VN, Völk HJ. 2003. Astron. Astrophys. 410:189-198

61. Berg TAM, Ellison SL, Venn KA, Prochaska JX. 2013. MNRAS 434:2892-2906

62. Binns WR, Wiedenbeck ME, Arnould M, Cummings AC, de Nolfo GA, et al. 2007. Space Sci. Rev. 130:439-449

63. Black JH. 1998. Faraday Discussions 109:257

64. Black JH, Fazio GG. 1973. Astrophys. J. 185:L7

65. Blandford R, Simeon P, Yuan Y. 2014. ArXiv e-prints

66. Blasi P. 2013. A\& A Rev. 21:70

67. Blitz L, Bazell D, Desert FX. 1990. Astrophys. J. 352:L13-L16

68. Boettcher E, Zweibel EG, Yoast-Hull TM, Gallagher III JS. 2013. Astrophys. J. 779:12

69. Bohlin RC, Savage BD, Drake JF. 1978. Astrophys. J. 224:132-142

70. Bolatto AD, Wolfire M, Leroy AK. 2013. Annu. Rev. Astron. Astrophys. 51:207268 
71. Bouchet L, Strong AW, Porter TA, Moskalenko IV, Jourdain E, Roques JP. 2011. Astrophys. J. 739:29

72. Breitschwerdt D, Dogiel VA, Völk HJ. 2002. Astron. Astrophys. 385:216-238

73. Brittain SD, Simon T, Kulesa C, Rettig TW. 2004. Astrophys. J. 606:911-916

74. Brooks AM, Venn KA, Lambert DL, Lemke M, Cunha K, Smith VV. 2002. Astrophys. J. 573:584-596

75. Büsching I, Kopp A, Pohl M, Schlickeiser R, Perrot C, Grenier I. 2005. Astrophys. J. 619:314-326

76. Bykov AM, Toptygin IN. 2001. Astronomy Letters 27:625-633

77. Caprioli D, Spitkovsky A. 2014. Astrophys. J. 783:91

78. Cardillo M, Tavani M, Giuliani A, Yoshiike S, Sano H, et al. 2014. Astron. Astrophys. 565:A74

79. Carlson E, Profumo S. 2014. Phys. Rev. D. 90:023015

80. Carretti E, Crocker RM, Staveley-Smith L, Haverkorn M, Purcell C, et al. 2013. Nature 493:66-69

81. Casandjian JM. 2012. In American Institute of Physics Conference Series, eds. FA Aharonian, W Hofmann, FM Rieger, vol. 1505 of American Institute of Physics Conference Series

82. Casse F, Lemoine M, Pelletier G. 2002. Phys. Rev. D. 65:023002

83. Castellina A, Donato F. 2013. Astrophysics of Galactic Charged Cosmic Rays. 725

84. Ceccarelli C, Hily-Blant P, Montmerle T, Dubus G, Gallant Y, Fiasson A. 2011. Astrophys. J. 740:L4

85. Cecchi-Pestellini C, Aiello S. 1992. MNRAS 258:125-133

86. Cesarsky CJ, Volk HJ. 1978. Astron. Astrophys. 70:367

87. Chang J, Adams JH, Ahn HS, Bashindzhagyan GL, Christl M, et al. 2008. Nature 456:362-365

88. Chernyakova M, Malyshev D, Aharonian FA, Crocker RM, Jones DI. 2011. Astrophys. J. 726:60

89. Combet C, Maurin D, Donnelly J, O'C. Drury L, Vangioni-Flam E. 2005. Astron. Astrophys. 435:151-160

90. Consolandi Ct. 2014. ArXiv e-prints

91. Coste B, Derome L, Maurin D, Putze A. 2012. Astron. Astrophys. 539:A88

92. Cotten DL, Magnani L. 2013. MNRAS 436:1152-1160

93. Crabtree KN, Indriolo N, Kreckel H, Tom BA, McCall BJ. 2011. Astrophys. J. $729: 15$

94. Crocker RM, Bicknell GV, Carretti E, Hill AS, Sutherland RS. 2014. Astrophys. J. 791:L20

95. Crocker RM, Jones DI, Aharonian F, Law CJ, Melia F, et al. 2011. MNRAS 413:763-788

96. Dalgarno A, Yan M, Liu W. 1999. Astrophys. J. Suppl. 125:237-256

97. Dame TM, Hartmann D, Thaddeus P. 2001. Astrophys. J. 547:792-813 
98. Dartois E, Ding JJ, de Barros ALF, Boduch P, Brunetto R, et al. 2013. Astron. Astrophys. 557:A97

99. Dermer CD, Finke JD, Murphy RJ, Strong AW, Loparco F, et al. 2013a. ArXiv e-prints

100. Dermer CD, Strong AW, Orlando E, Tibaldo L, for the Fermi Collaboration. 2013b. ArXiv e-prints

101. Di Bernardo G, Evoli C, Gaggero D, Grasso D, Maccione L. 2013. JCAP 3:36

102. Dickey JM, Strasser S, Gaensler BM, Haverkorn M, Kavars D, et al. 2009. Astrophys. J. 693:1250-1260

103. Digel SW, Grenier IA, Hunter SD, Dame TM, Thaddeus P. 2001. Astrophys. J. $555: 12-22$

104. Draine BT. 2011. Physics of the Interstellar and Intergalactic Medium. Princeton: Princeton University Press

105. Drury LO, Aharonian FA, Voelk HJ. 1994. Astron. Astrophys. 287:959-971

106. Ellison DC, Bykov AM. 2011. Astrophys. J. 731:87

107. Ellison DC, Drury LO, Meyer JP. 1997. Astrophys. J. 487:197-217

108. Etxaluze M, Cernicharo J, Goicoechea JR, van Hoof PAM, Swinyard BM, et al. 2014. Astron. Astrophys. 566:A78

109. Everett J, Zweibel E, Benjamin B, McCammon D, Schiller Q, et al. 2012. In EAS Publications Series, ed. MA de Avillez, vol. 56 of EAS Publications Series

110. Everett JE, Zweibel EG. 2011. Astrophys. J. 739:60

111. Evoli C, Gaggero D, Grasso D, Maccione L. 2012. Physical Review Letters 108:211102

112. Evoli C, Yan H. 2014. Astrophys. J. 782:36

113. Falgarone E, Godard B, Cernicharo J, de Luca M, Gerin M, et al. 2010a. Astron. Astrophys. 521:L15

114. Falgarone E, Ossenkopf V, Gerin M, Lesaffre P, Godard B, et al. 2010b. Astron. Astrophys. 518:L118

115. Fang J, Yu H, Zhu BT, Zhang L. 2013. MNRAS 435:570-574

116. Fang J, Zhang L. 2013. New Astron. 18:35-41

117. Farrar GR. 2014. Comptes Rendus Physique 15:339-348

118. Fatuzzo M, Melia F. 2014. Astrophys. J. 784:131

119. Fatuzzo M, Melia F, Todd E, Adams FC. 2010. Astrophys. J. 725:515-527

120. Fenech DM, Muxlow TWB, Beswick RJ, Pedlar A, Argo MK. 2008. MNRAS 391:1384-1402

121. Ferrand G, Marcowith A. 2010. Astron. Astrophys. 510:A101

122. Fletcher A, Beck R, Shukurov A, Berkhuijsen EM, Horellou C. 2011. MNRAS 412:2396-2416

123. Fox AJ, Bordoloi R, Savage BD, Lockman FJ, Jenkins EB, et al. 2014. ArXiv e-prints

124. Fukui Y, Torii K, Onishi T, Yamamoto H, Okamoto R, et al. 2014. ArXiv e-prints

125. Fuskeland U, Wehus IK, Eriksen HK, Næss SK. 2014. Astrophys. J. 790:104 
126. Gaggero D, Maccione L, Grasso D, Di Bernardo G, Evoli C. 2014. Phys. Rev. D. 89:083007

127. Galeazzi M, Chiao M, Collier MR, Cravens T, Koutroumpa D, et al. 2014. Nature 512:171-173

128. Gargaté L, Spitkovsky A. 2012. Astrophys. J. 744:67

129. Geballe TR. 2012. Royal Society of London Philosophical Transactions Series A 370:5151-5161

130. Geballe TR, McCall BJ, Hinkle KH, Oka T. 1999. Astrophys. J. 510:251-257

131. Geballe TR, Oka T. 1996. Nature 384:334-335

132. Gendre MA, Fenech DM, Beswick RJ, Muxlow TWB, Argo MK. 2013. MNRAS 431:1107-1120

133. Gerakines PA, Moore MH, Hudson RL. 2001. JGR 106:33381-33386

134. Gerin M, de Luca M, Black J, Goicoechea JR, Herbst E, et al. 2010. Astron. Astrophys. 518:L110

135. Giacalone J, Jokipii JR. 1994. Astrophys. J. 430:L137-L140

136. Giacalone J, Jokipii JR. 1999. Astrophys. J. 520:204-214

137. Gibb EL, Brittain SD, Rettig TW, Troutman M, Simon T, Kulesa C. 2010. Astrophys. J. 715:757-766

138. Girichidis P, Naab T, Walch S, Hanasz M. 2014. ArXiv e-prints

139. Glover SCO, Clark PC. 2012. MNRAS 421:116-131

140. Glover SCO, Mac Low MM. 2011. MNRAS 412:337-350

141. Godard B, Falgarone E, Gerin M, Lis DC, De Luca M, et al. 2012. Astron. Astrophys. 540:A87

142. Godard B, Falgarone E, Pineau Des Forêts G. 2009. Astron. Astrophys. 495:847867

143. Godard B, Falgarone E, Pineau des Forêts G. 2014. Astron. Astrophys. 570:A27

144. González-Alfonso E, Fischer J, Bruderer S, Müller HSP, Graciá-Carpio J, et al. 2013. Astron. Astrophys. 550:A25

145. Goto M, Geballe TR, Indriolo N, Yusef-Zadeh F, Usuda T, et al. 2014. Astrophys. J. $786: 96$

146. Goto M, Indriolo N, Geballe TR, Usuda T. 2013. Journal of Physical Chemistry A 117:9919-9930

147. Goto M, McCall BJ, Geballe TR, Usuda T, Kobayashi N, et al. 2002. Publ. Astron. Soc. Jpn. 54:951-961

148. Goto M, Usuda T, Geballe TR, Indriolo N, McCall BJ, et al. 2011. Publ. Astron. Soc. Jpn. 63:L13-L17

149. Goto M, Usuda T, Nagata T, Geballe TR, McCall BJ, et al. 2008. Astrophys. J. 688:306-319

150. Gredel R, Lepp S, Dalgarno A, Herbst E. 1989. Astrophys. J. 347:289-293

151. Grenier IA. 2000. Astron. Astrophys. 364:L93-L96

152. Grenier IA, Casandjian JM, Terrier R. 2005. Science 307:1292-1295 
153. Gupta H, Rimmer P, Pearson JC, Yu S, Herbst E, et al. 2010. Astron. Astrophys. 521:L47

154. Hams T, Barbier LM, Bremerich M, Christian ER, de Nolfo GA, et al. 2004. Astrophys. J. 611:892-905

155. Hanabata Y, Katagiri H, Hewitt JW, Ballet J, Fukazawa Y, et al. 2014. Astrophys. J. $786: 145$

156. Hanasz M, Kowalik K, Wóltański D, Pawłaszek R. 2010. In EAS Publications Series, eds. K Gożdziewski, A Niedzielski, J Schneider, vol. 42 of EAS Publications Series

157. Hanasz M, Kowalik K, Wóltański D, Pawłaszek R. 2012a. In EAS Publications Series, ed. MA de Avillez, vol. 56 of EAS Publications Series

158. Hanasz M, Otmianowska-Mazur K, Kowal G, Lesch H. 2009. Astron. Astrophys. 498:335-346

159. Hanasz M, Otmianowska-Mazur K, Lesch H, Kowal G, Soida M, et al. 2012b. In EAS Publications Series, ed. MA de Avillez, vol. 56 of EAS Publications Series

160. Haverkorn M. 2015. In Astrophysics and Space Science Library, eds. A Lazarian, EM de Gouveia Dal Pino, C Melioli, vol. 407 of Astrophysics and Space Science Library

161. Heays AN, Visser R, Gredel R, Ubachs W, Lewis BR, et al. 2014. Astron. Astrophys. 562:A61

162. Hebrard G, Lemoine M, Ferlet R, Vidal-Madjar A. 1997. Astron. Astrophys. 324:1145-1148

163. Helou G, Soifer BT, Rowan-Robinson M. 1985. Astrophys. J. 298:L7-L11

164. Herbst E, Klemperer W. 1973. Astrophys. J. 185:505-534

165. Higdon JC, Lingenfelter RE. 2005. Astrophys. J. 628:738-749

166. Hollenbach D, Kaufman MJ, Neufeld D, Wolfire M, Goicoechea JR. 2012. Astrophys. J. 754:105

167. Howk JC, Lehner N, Fields BD, Mathews GJ. 2012. Nature 489:121-123

168. Howk JC, Sembach KR, Savage BD. 2000. Astrophys. J. 543:278-283

169. Hunter SD, Bertsch DL, Catelli JR, Dame TM, Digel SW, et al. 1997. Astrophys. J. 481:205-240

170. Indriolo N. 2012. Royal Society of London Philosophical Transactions Series A 370:5142-5150

171. Indriolo N, Blake GA, Goto M, Usuda T, Oka T, et al. 2010. Astrophys. J. 724:1357-1365

172. Indriolo N, Geballe TR, Oka T, McCall BJ. 2007. Astrophys. J. 671:1736-1747

173. Indriolo N, McCall BJ. 2012. Astrophys. J. 745:91

174. Indriolo N, McCall BJ. 2013. Chem. Soc. Rev. 42:7763-7773

175. Indriolo N, Neufeld DA, Gerin M, Geballe TR, Black JH, et al. 2012. Astrophys. J. $758: 83$

176. Indriolo N, Neufeld DA, Gerin M, Schilke P, Benz AO, et al. 2014. Astrophys. J. submitted 
177. Ingleby L, Calvet N, Bergin E, Yerasi A, Espaillat C, et al. 2009. Astrophys. J. 703:L137-L141

178. Irwin J, Beck R, Benjamin RA, Dettmar RJ, English J, et al. 2012a. Astron. J. 144:43

179. Irwin J, Beck R, Benjamin RA, Dettmar RJ, English J, et al. 2012b. Astron. J. 144:44

180. Irwin J, Krause M, English J, Beck R, Murphy E, et al. 2013. Astron. J. 146:164

181. Jaffe TR, Banday AJ, Leahy JP, Leach S, Strong AW. 2011. MNRAS 416:11521162

182. Jansson R, Farrar GR. 2012a. Astrophys. J. 757:14

183. Jansson R, Farrar GR. 2012b. Astrophys. J. 761:L11

184. Jonin C, Liu X, Ajello JM, James GK, Abgrall H. 2000. Astrophys. J. Suppl. 129:247-266

185. Kalberla PMW, Dedes L. 2008. Astron. Astrophys. 487:951-963

186. Kamenetzky J, Glenn J, Rangwala N, Maloney P, Bradford M, et al. 2012. Astrophys. J. 753:70

187. Kappl R, Winkler MW. 2014. JCAP 9:51

188. Kawanomoto S, Kajino T, Aoki W, Bessell M, Suzuki TK, et al. 2009. Astrophys. J. 701:1506-1518

189. Kissmann R. 2014. Astroparticle Physics 55:37-50

190. Knauth DC, Federman SR, Lambert DL. 2003. Astrophys. J. 586:268-285

191. Knauth DC, Federman SR, Lambert DL, Crane P. 2000. Nature 405:656-658

192. Koldobskiy SA, Adriani O, Barbarino GC, Bazilevskaya GA, Bellotti R, et al. 2013. Journal of Physics Conference Series 409:012040

193. Korn AJ, Becker SR, Gummersbach CA, Wolf B. 2000. Astron. Astrophys. 353:655-665

194. Kotera K, Olinto AV. 2011. Annu. Rev. Astron. Astrophys. 49:119-153

195. Krełowski J, Beletsky Y, Galazutdinov GA. 2010. Astrophys. J. 719:L20-L22

196. Kulesa CA. 2002. Molecular hydrogen and its ions in dark interstellar clouds and star forming regions. Ph.D. thesis, The University of Arizona

197. Lacki BC. 2014. MNRAS 444:L39-L43

198. Lallement R, Vergely JL, Valette B, Puspitarini L, Eyer L, Casagrande L. 2014. Astron. Astrophys. 561:A91

199. Lambert DL, Sheffer Y, Federman SR, Cardelli JA, Sofia UJ, Knauth DC. 1998. Astrophys. J. 494:614-622

200. Langer WD, Velusamy T, Pineda JL, Willacy K, Goldsmith PF. 2014. Astron. Astrophys. 561:A122

201. Lavalle J, Maurin D, Putze A. 2014. Phys. Rev. D. 90:081301

202. Lave KA, Wiedenbeck ME, Binns WR, Christian ER, Cummings AC, et al. 2013. Astrophys. J. 770:117

203. Law CJ. 2010. Astrophys. J. 708:474-484

204. Lazarian A, Yan H. 2014. Astrophys. J. 784:38 
205. Lebrun F, Paul JA. 1983. Astrophys. J. 266:276-286

206. Lee MY, Stanimirović S, Wolfire MG, Shetty R, Glover SCO, et al. 2014. Astrophys. J. $784: 80$

207. Levrier F, Le Petit F, Hennebelle P, Lesaffre P, Gerin M, Falgarone E. 2012. Astron. Astrophys. 544:A22

208. Lineros RA, Bringmann T, Donato F. 2012. Journal of Physics Conference Series 375:012037

209. Lingenfelter RE, Higdon JC. 2007. Astrophys. J. 660:330-335

210. Lis DC, Schilke P, Bergin EA, Gerin M, Black JH, et al. 2014. Astrophys. J. $785: 135$

211. Liszt H. 2014. Astrophys. J. 780:10

212. Liszt HS, Pety J, Lucas R. 2010. Astron. Astrophys. 518:A45

213. Lonsdale CJ, Diamond PJ, Thrall H, Smith HE, Lonsdale CJ. 2006. Astrophys. J. $647: 185-193$

214. Lorimer DR, Faulkner AJ, Lyne AG, Manchester RN, Kramer M, et al. 2006. MNRAS 372:777-800

215. Malkov MA, Diamond PH, Sagdeev RZ, Aharonian FA, Moskalenko IV. 2013. Astrophys. J. 768:73

216. Martin PG, Roy A, Bontemps S, Miville-Deschênes MA, Ade PAR, et al. 2012. Astrophys. J. 751:28

217. Maurin D, Melot F, Taillet R. 2014. Astron. Astrophys. 569:A32

218. Maurin D, Putze A, Derome L. 2010. Astron. Astrophys. 516:A67

219. Mayorov AG, Adriani O, Barbarino GC, Bazilevskaya GA, Bellotti R, et al. 2013. Journal of Physics Conference Series 409:012056

220. McCall BJ, Geballe TR, Hinkle KH, Oka T. 1998. Science 279:1910

221. McCall BJ, Geballe TR, Hinkle KH, Oka T. 1999. Astrophys. J. 522:338-348

222. McCall BJ, Hinkle KH, Geballe TR, Moriarty-Schieven GH, Evans II NJ, et al. 2002. Astrophys. J. 567:391-406

223. McCall BJ, Huneycutt AJ, Saykally RJ, Geballe TR, Djuric N, et al. 2003. Nature 422:500-502

224. McElroy D, Walsh C, Markwick AJ, Cordiner MA, Smith K, Millar TJ. 2013. Astron. Astrophys. 550:A36

225. Mertsch P, Sarkar S. 2013. JCAP 6:41

226. Mertsch P, Sarkar S. 2014. Phys. Rev. D. 90:061301

227. Mikhailov V, Adriani O, Barbarino G, Bazilevskaya GA, Bellotti R, et al. 2013. Journal of Physics Conference Series 409:012035

228. Moskalenko I. 2011. International Cosmic Ray Conference 6:283

229. Moskalenko IV, Porter TA, Digel SW. 2006. Astrophys. J. 652:L65-L68

230. Muñoz Caro GM, Dartois E, Boduch P, Rothard H, Domaracka A, JiménezEscobar A. 2014. Astron. Astrophys. 566:A93

231. Mulcahy DD, Horneffer A, Beck R, Heald G, Fletcher A, et al. 2014. Astron. Astrophys. 568:A74 
232. Murphy EJ, Porter TA, Moskalenko IV, Helou G, Strong AW. 2012. Astrophys. J. $750: 126$

233. Nakamura K, Yoshida T, Shigeyama T, Kajino T. 2010. Astrophys. J. 718:L137L140

234. Nava L, Gabici S. 2013. MNRAS 429:1643-1651

235. Neufeld DA, Goicoechea JR, Sonnentrucker P, Black JH, Pearson J, et al. 2010. Astron. Astrophys. 521:L10

236. Obermeier A, Ave M, Boyle P, Höppner C, Hörandel J, Müller D. 2011. Astrophys. J. $742: 14$

237. Oka T, Epp E. 2004. Astrophys. J. 613:349-354

238. Oka T, Geballe TR, Goto M, Usuda T, McCall BJ. 2005. Astrophys. J. 632:882893

239. Orlando E, Strong A. 2013. MNRAS 436:2127-2142

240. Orlando E, Strong AW. 2007. Astrophys. Sp. Sci. 309:359-363

241. Orlando E, Strong AW. 2008. Astron. Astrophys. 480:847-857

242. Ormes JF, ed. 2013. Centenary symposium 2012: discovery of cosmic rays, vol. 1516 of American Institute of Physics Conference Series

243. Padovani M, Galli D. 2011. Astron. Astrophys. 530:A109

244. Padovani M, Galli D, Glassgold AE. 2009. Astron. Astrophys. 501:619-631

245. Padovani M, Galli D, Glassgold AE. 2013. Astron. Astrophys. 549:C3

246. Padovani M, Hennebelle P, Galli D. 2013. Astron. Astrophys. 560:A114

247. Paglione TAD, Abrahams RD. 2012. Astrophys. J. 755:106

248. Palumbo ME, Baratta GA, Fulvio D, Garozzo M, Gomis O, et al. 2008. Journal of Physics Conference Series 101:012002

249. Pan FS, Oka T. 1986. Astrophys. J. 305:518-525

250. Panov AD, Adams JH, Ahn HS, Bashinzhagyan GL, Watts JW, et al. 2009. Bulletin of the Russian Academy of Science, Phys. 73:564-567

251. Paradis D, Dobashi K, Shimoikura T, Kawamura A, Onishi T, et al. 2012. Astron. Astrophys. 543:A103

252. Parizot E, Marcowith A, van der Swaluw E, Bykov AM, Tatischeff V. 2004. Astron. Astrophys. 424:747-760

253. Parra R, Conway JE, Diamond PJ, Thrall H, Lonsdale CJ, et al. 2007. Astrophys. J. 659:314-330

254. Pereira-Santaella M, Spinoglio L, Busquet G, Wilson CD, Glenn J, et al. 2013. Astrophys. J. 768:55

255. Perrot CA, Grenier IA. 2003. Astron. Astrophys. 404:519-531

256. Pineda JL, Langer WD, Velusamy T, Goldsmith PF. 2013. Astron. Astrophys. 554:A103

257. Planck, Fermi Collaborations, :, Ade PAR, Aghanim N, et al. 2014. ArXiv eprints

258. Planck Collaboration, Abergel A, Ade PAR, Aghanim N, Alves MIR, et al. 2014a. Astron. Astrophys. 571:A11 
259. Planck Collaboration, Abergel A, Ade PAR, Aghanim N, Alves MIR, et al. 2014b. Astron. Astrophys. 566:A55

260. Planck Collaboration, Abergel A, Ade PAR, Aghanim N, Arnaud M, et al. 2011a. Astron. Astrophys. 536:A21

261. Planck Collaboration, Ade PAR, Aghanim N, Arnaud M, Ashdown M, et al. 2011b. Astron. Astrophys. 536:A19

262. Pohl M, Eichler D. 2013. Astrophys. J. 766:4

263. Porras AJ, Federman SR, Welty DE, Ritchey AM. 2014. Astrophys. J. 781:L8

264. Prantzos N. 2012. Astron. Astrophys. 538:A80

265. Prasad SS, Tarafdar SP. 1983. Astrophys. J. 267:603-609

266. Prochaska JX, Howk JC, Wolfe AM. 2003. Nature 423:57-59

267. Ptuskin V, Zirakashvili V, Seo ES. 2013. Astrophys. J. 763:47

268. Ptuskin VS, Moskalenko IV, Jones FC, Strong AW, Zirakashvili VN. 2006. Astrophys. J. 642:902-916

269. Ptuskin VS, Zirakashvili VN, Plesser AA. 2008. Advances in Space Research 42:486-490

270. Putze A, Derome L, Maurin D. 2010. Astron. Astrophys. 516:A66

271. Putze A, Maurin D, Donato F. 2011. Astron. Astrophys. 526:A101

272. Rampadarath H, Morgan JS, Lenc E, Tingay SJ. 2014. Astron. J. 147:5

273. Rangwala N, Maloney PR, Glenn J, Wilson CD, Rykala A, et al. 2011. Astrophys. J. $743: 94$

274. Rauch BF, Link JT, Lodders K, Israel MH, Barbier LM, et al. 2009. Astrophys. J. 697:2083-2088

275. Rimmer PB, Herbst E, Morata O, Roueff E. 2012. Astron. Astrophys. 537:A7

276. Ritchey AM, Federman SR, Sheffer Y, Lambert DL. 2011. Astrophys. J. 728:70

277. Roy A, Martin PG, Polychroni D, Bontemps S, Abergel A, et al. 2013. Astrophys. J. $763: 55$

278. Salmon S, Montalbán J, Morel T, Miglio A, Dupret MA, Noels A. 2012. MNRAS 422:3460-3474

279. Sandstrom KM, Leroy AK, Walter F, Bolatto AD, Croxall KV, et al. 2013. Astrophys. J. $777: 5$

280. Sato M, Wu YW, Immer K, Zhang B, Sanna A, et al. 2014. Astrophys. J. 793:72

281. Schilke P, Comito C, Müller HSP, Bergin EA, Herbst E, et al. 2010. Astron. Astrophys. 521:L11

282. Schilke P, Neufeld DA, Müller HSP, Comito C, Bergin EA, et al. 2014. Astron. Astrophys. 566:A29

283. Schlickeiser R, Webber WR, Kempf A. 2014. Astrophys. J. 787:35

284. Schwadron NA, Adams FC, Christian ER, Desiati P, Frisch P, et al. 2014. Science 343:988-990

285. Seperuelo Duarte E, Boduch P, Rothard H, Been T, Dartois E, et al. 2009. Astron. Astrophys. 502:599-603 
286. Sheffer Y, Rogers M, Federman SR, Abel NP, Gredel R, et al. 2008. Astrophys. J. $687: 1075-1106$

287. Shen CJ, Greenberg JM, Schutte WA, van Dishoeck EF. 2004. Astron. Astrophys. 415:203-215

288. Skilling J, Strong AW. 1976. Astron. Astrophys. 53:253-258

289. Slane P, Bykov A, Ellison DC, Dubner G, Castro D. 2014. Space Sci. Rev.

290. Snow TP, McCall BJ. 2006. Annu. Rev. Astron. Astrophys. 44:367-414

291. Spinoglio L, Pereira-Santaella M, Busquet G, Schirm MRP, Wilson CD, et al. 2012. Astrophys. J. 758:108

292. Spitkovsky A. 2008. Astrophys. J. 682:L5-L8

293. Spitzer Jr. L, Tomasko MG. 1968. Astrophys. J. 152:971

294. Sreekumar P, Bertsch DL, Dingus BL, Fichtel CE, Hartman RC, et al. 1993. Physical Review Letters 70:127-129

295. Sternberg A, Dalgarno A, Lepp S. 1987. Astrophys. J. 320:676-682

296. Stone EC, Cummings AC, McDonald FB, Heikkila BC, Lal N, Webber WR. 2013. Science 341:150-153

297. Strong AW. 2007. Astrophys. Sp. Sci. 309:35-41

298. Strong AW. 2011. In Cosmic Rays for Particle and Astroparticle Physics, eds. S Giani, C Leroy, PG Rancoita

299. Strong AW, Bloemen JBGM, Dame TM, Grenier IA, Hermsen W, et al. 1988. Astron. Astrophys. 207:1-15

300. Strong AW, Moskalenko IV. 1998. Astrophys. J. 509:212-228

301. Strong AW, Moskalenko IV. 2009. ArXiv e-prints

302. Strong AW, Moskalenko IV, Ptuskin VS. 2007. Annual Review of Nuclear and Particle Science 57:285-327

303. Strong AW, Orlando E, Jaffe TR. 2011. Astron. Astrophys. 534:A54

304. Strong AW, Porter TA, Digel SW, Jóhannesson G, Martin P, et al. 2010. Astrophys. J. 722:L58-L63

305. Su M, Slatyer TR, Finkbeiner DP. 2010. Astrophys. J. 724:1044-1082

306. Sun XH, Reich W, Waelkens A, Enßlin TA. 2008. Astron. Astrophys. 477:573-592

307. Taillet R, Maurin D. 2003. Astron. Astrophys. 402:971-983

308. Tang X, Chevalier RA. 2014. Astrophys. J. 784:L35

309. Taylor CJ, Ritchey AM, Federman SR, Lambert DL. 2012. Astrophys. J. 750:L15

310. Telezhinsky I, Dwarkadas VV, Pohl M. 2012. Astron. Astrophys. 541:A153

311. The Fermi LAT collaboration, Ackermann M, Ajello M, Albert A, Atwood WB, et al. 2014. ArXiv e-prints

312. Thornbury A, Drury LO. 2014. MNRAS 442:3010-3012

313. Tielens AGGM. 2005. The Physics and Chemistry of the Interstellar Medium. Cambridge, UK: Cambridge University Press

314. Trotta R, Jóhannesson G, Moskalenko IV, Porter TA, Ruiz de Austri R, Strong AW. 2011. Astrophys. J. 729:106 
315. Uchiyama Y, Blandford RD, Funk S, Tajima H, Tanaka T. 2010. Astrophys. J. 723:L122-L126

316. Uhlig M, Pfrommer C, Sharma M, Nath BB, Enßlin TA, Springel V. 2012. MN$R A S$ 423:2374-2396

317. Urquhart JS, Figura CC, Moore TJT, Hoare MG, Lumsden SL, et al. 2014. MNRAS 437:1791-1807

318. van der Tak FFS, Nagy Z, Ossenkopf V, Makai Z, Black JH, et al. 2013. Astron. Astrophys. 560:A95

319. van der Werf PP, Isaak KG, Meijerink R, Spaans M, Rykala A, et al. 2010. Astron. Astrophys. 518:L42

320. van Dishoeck EF, Black JH. 1988. Astrophys. J. 334:771-802

321. Vaupré S, Hily-Blant P, Ceccarelli C, Dubus G, Gabici S, Montmerle T. 2014. Astron. Astrophys. 568:A50

322. VERITAS Collaboration, Acciari VA, Aliu E, Arlen T, Aune T, et al. 2009. Nature 462:770-772

323. Visser R, van Dishoeck EF, Black JH. 2009. Astron. Astrophys. 503:323-343

324. Vladimirov AE, Jóhannesson G, Moskalenko IV, Porter TA. 2012. Astrophys. J. 752:68

325. Voelk HJ. 1989. Astron. Astrophys. 218:67-70

326. Völk HJ, Berezhko EG. 2013. Astrophys. J. 777:149

327. Waelkens A, Jaffe T, Reinecke M, Kitaura FS, Enßlin TA. 2009. Astron. Astrophys. 495:697-706

328. Wakelam V, Herbst E, Loison JC, Smith IWM, Chandrasekaran V, et al. 2012. Astrophys. J. Suppl. 199:21

329. Welty DE, Ritchey AM, Dahlstrom JA, York DG. 2014. Astrophys. J. 792:106

330. Werner M, Kissmann R, Strong AW, Reimer O. 2013. ArXiv e-prints

331. Werner M, Kissmann R, Strong AW, Reimer O. 2014. ArXiv e-prints

332. Wiedenbeck ME, Binns WR, Christian ER, Cummings AC, Dougherty BL, et al. 1999. Astrophys. J. 523:L61-L64

333. Wolfire MG, Hollenbach D, McKee CF. 2010. Astrophys. J. 716:1191-1207

334. Wóltański D, Hanasz M, Kowalik K. 2013. In Cosmic Rays in Star-Forming Environments, eds. DF Torres, O Reimer, vol. 34 of Advances in Solid State Physics

335. Woosley SE, Hartmann DH, Hoffman RD, Haxton WC. 1990. Astrophys. J. 356:272-301

336. Wyrowski F, Menten KM, Güsten R, Belloche A. 2010. Astron. Astrophys. 518:A26

337. Yan H, Lazarian A. 2008. Astrophys. J. 673:942-953

338. Yang HYK, Ruszkowski M, Zweibel E. 2013. MNRAS 436:2734-2746

339. Yoast-Hull TM, Everett JE, Gallagher III JS, Zweibel EG. 2013a. Astrophys. J. 768:53

340. Yoast-Hull TM, Everett JE, Gallagher III JS, Zweibel EG. 2013b. Astrophys. J. 768:53 
341. Yoast-Hull TM, Gallagher III JS, Zweibel EG. 2014. Astrophys. J. 790:86

342. Yoast-Hull TM, Gallagher III JS, Zweibel EG, Everett JE. 2014. Astrophys. J. 780:137

343. Yoon YS, Ahn HS, Allison PS, Bagliesi MG, Beatty JJ, et al. 2011. Astrophys. J. $728: 122$

344. Yuan Q, Zhang B. 2014. Journal of High Energy Astrophysics 3:1-8

345. Yusef-Zadeh F, Hewitt JW, Wardle M, Tatischeff V, Roberts DA, et al. 2013. Astrophys. J. 762:33

346. Zweibel EG. 2013. Physics of Plasmas 20:055501 


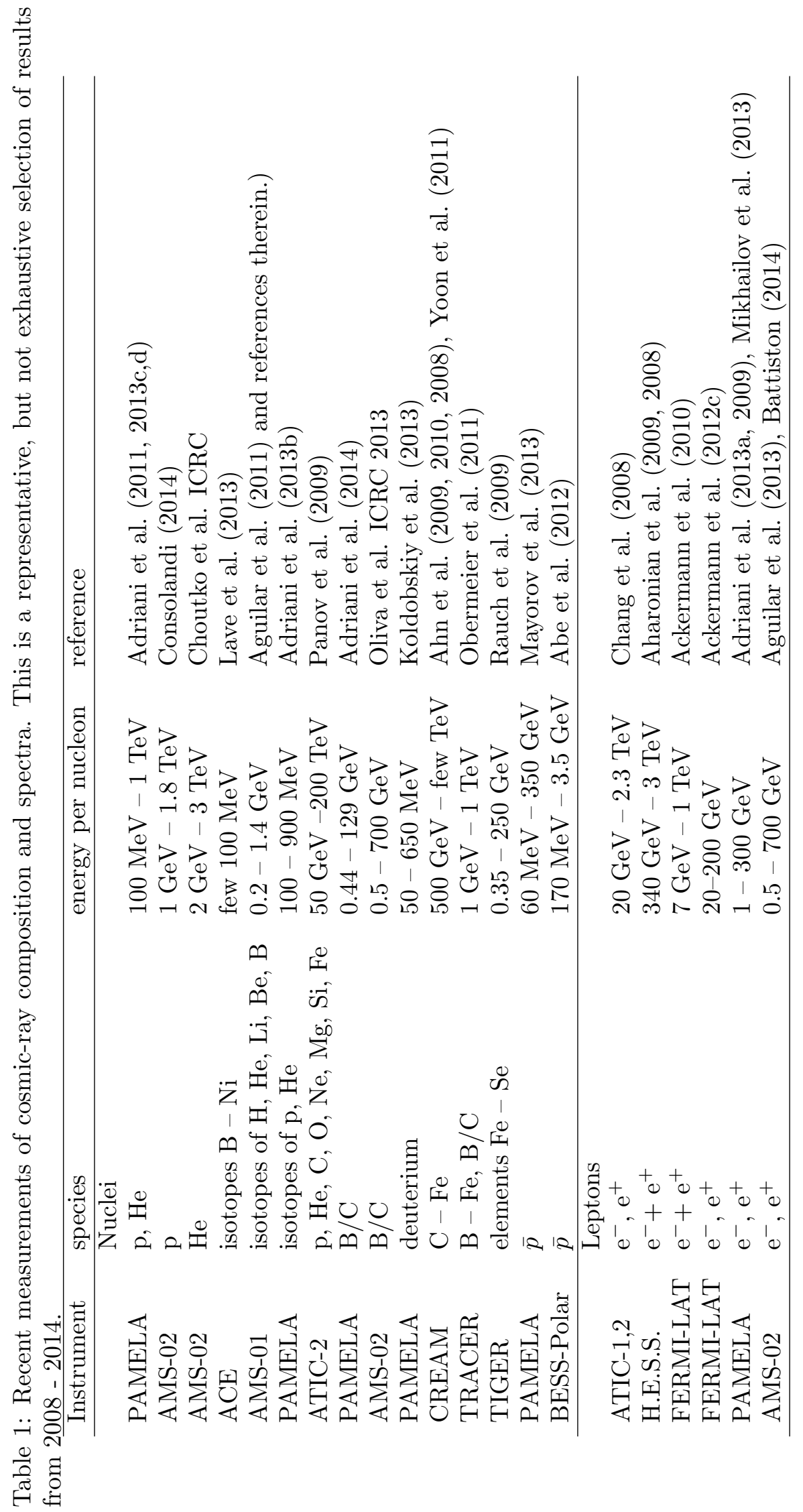




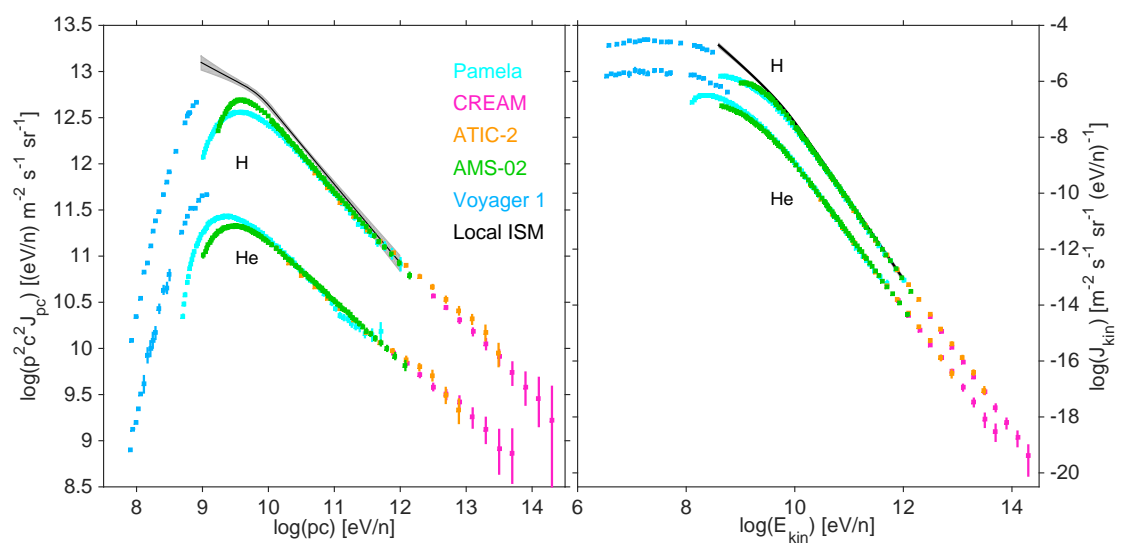

Figure 1: Local spectra of CR protons and Helium, as measured near the Earth and heliopause (Choutko et al. 2013 ICRC, Adriani et al. 2011, Consolandi 2014, Panov et al. 2009, Stone et al. 2013, Yoon et al. 2011), and displayed without solar demodulation in momentum distributions (left) and in particle spectra in kinetic energy (right). They indicate proton hardening and He enrichment above a few hundred $\mathrm{GeV}$. The grey band marks the range of proton spectra inferred in the local ISM from the average $\gamma$-ray emissivity of the interstellar gas, given the current uncertainties in the hadronic cross sections (Dermer et al. 2013b).

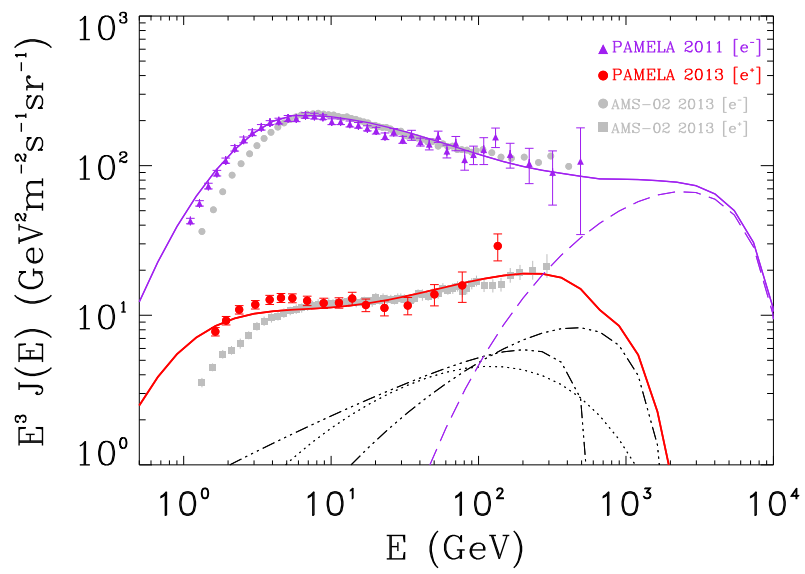

Figure 2: CR electron and positron spectra from AMS-02 and PAMELA, compared with 3D models with possible contributions from nearby pulsars (dashed line for Vela, triple dotted-dashed lines for Monogem and Geminga) and an additional component required to explain the data (from Gaggero et al. 2014). Solid lines show the total spectra including the Galactic primary and secondary leptons. Solar modulation is modelled to a level appropriate to the PAMELA data. 

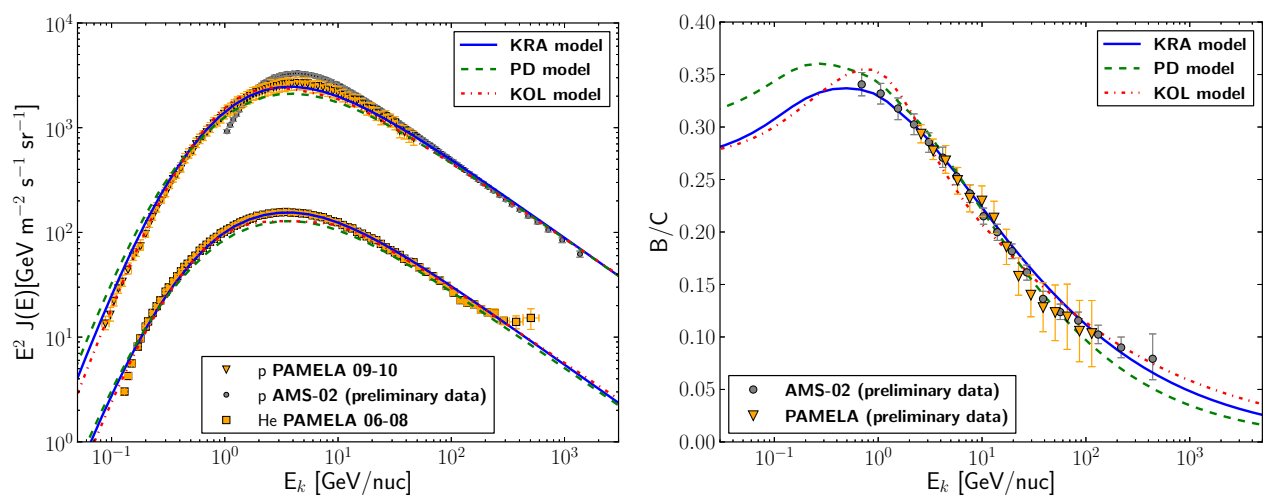

Figure 3: Left: proton and Helium spectra from AMS-02 and PAMELA, compared with propagation models which fit B/C. This shows how the primary injection spectrum combined with propagation can be modelled. Right: spectral evolution of the $\mathrm{B} / \mathrm{C}$ ratio from AMS-02 and PAMELA, compared with 3D propagation models, with various momentum-dependences of the diffusion coefficient (KRA: Kraichnan turbulence, KOL: Kolmogorov turbulence, PD: pure diffusion without reacceleration). This shows how such models can be used to interpret the data, in this case with a $3 \mathrm{D}$ treatment of diffusion, and spiral structure in the source distribution. Solar modulation, important below a few $\mathrm{GeV}$, is included in the predictions. From Gaggero et al. (2014). 


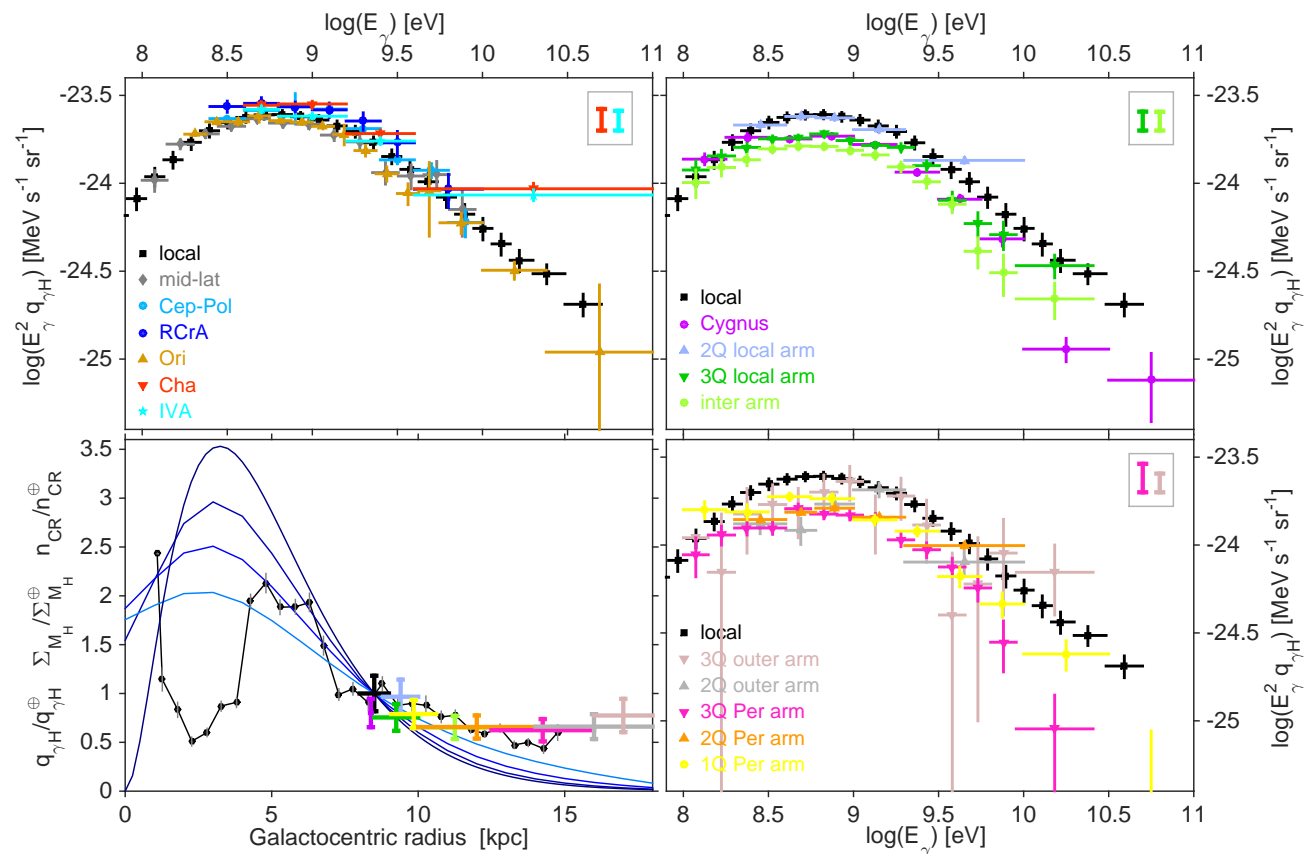

Figure 4: $\gamma$-ray emissivity spectra per interstellar $\mathrm{H}$ atom, $q_{\gamma \mathrm{H}}$, measured with Fermi LAT in the atomic gas of nearby clouds, in different sectors along the Local and Perseus Arms, and between these arms (Abdo et al. 2009, 2010g, Ackermann et al. 2012b,d,f, 2011b, Planck et al. 2014). The errors are statistical and the inserts illustrate the range of potential uncertainties due to Hi optical depth corrections and LAT exposure. The average spectrum found in the local ISM (in black), at $10^{\circ} \leq|b| \leq 70^{\circ}$, is common to the three spectral plots (Casandjian et al. 2013 ICRC). It includes systematic uncertainties. The lower left plot shows the radial distribution across the Milky Way of different quantities, relative to their value near the Sun: with cloud colours, $q_{\gamma \mathrm{H}}$ integrals above $400 \mathrm{MeV}$ as proxies for the CR densities (the errors include uncertainties in the LAT exposure and a $15 \%$ uncertainty in the derivation of $N_{\mathrm{HI}}$ column densities); in black, the mass surface density of the total gas (Pineda et al. 2013); from light to dark blue, CR densities modelled with GALPROP for halo heights of 10,4 , and $2 \mathrm{kpc}$, respectively, and the input CR source distribution following that of pulsars (model $\mathrm{C}$ of Lorimer et al. 2006). 

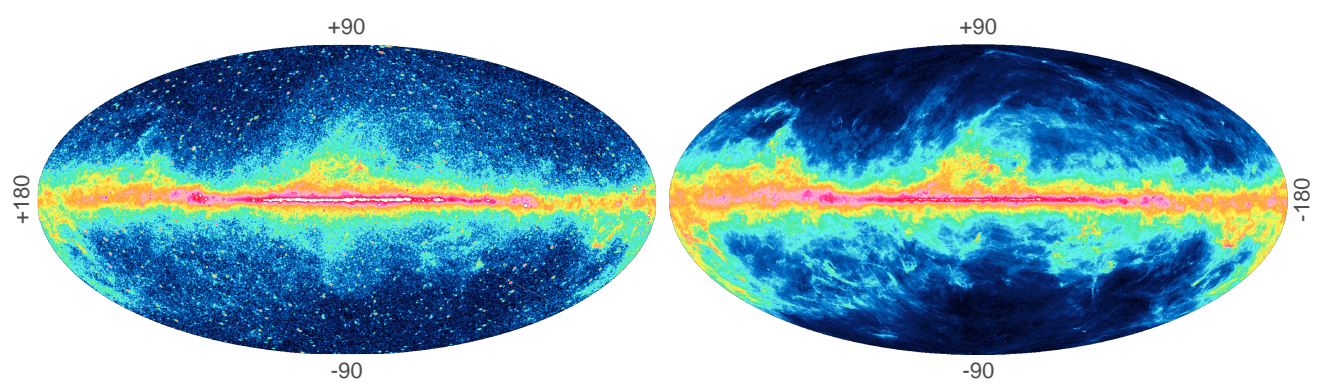

Figure 5: Skymaps of the $\gamma$-ray intensity recorded by Fermi-LAT above $1 \mathrm{GeV}$ in six years of observations (left, http://fermi.gsfc.nasa.gov/ssc/) and of the dust optical depth measured at $353 \mathrm{GHz}$ from the Planck and IRAS surveys (right, Planck Collaboration et al. 2014a). Both maps broadly trace the same total gas column densities, weighted by the ambient $\mathrm{CR}$ density in $\gamma$ rays, and by the ambient dust-to-gas mass ratio and starlight heating rate in the dust map. They exhibit striking similarities in details of the gas features. The $\gamma$-ray map also contains numerous point sources and faint non-gas related diffuse components.

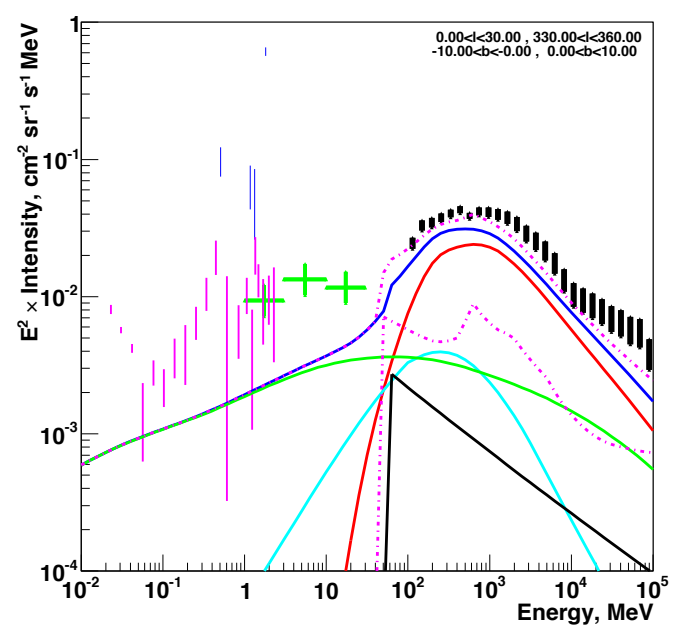

Figure 6: $\gamma$-ray spectrum of the inner Galaxy from INTEGRAL-SPI (magenta and blue bars), CGRO-COMPTEL (green bars), and Fermi-LAT (black bars), compared with the radiation output from CRs in the ISM (pion-decay in solid red, inverse Compton in green, bremsstrahlung in cyan). Other contributors are the isotropic background for Fermi-LAT (black power law) and detected sources (lower red dotted curve). The total model is given with (upper red dotted line) and without (blue) sources. From Strong (2011). 


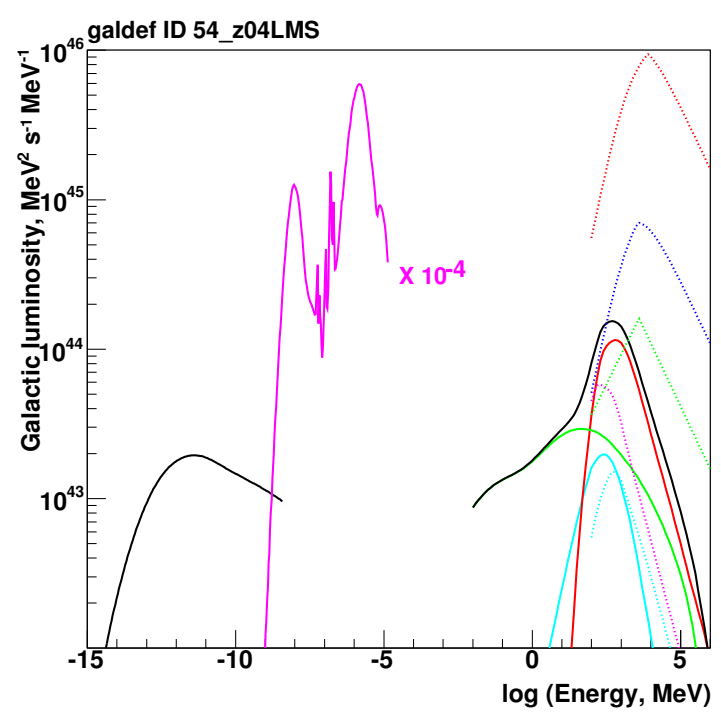

Figure 7: Multi-wavelength luminosity spectrum of the Milky Way Galaxy, from radio to $\gamma$ rays, and including CRs: synchrotron emission (left black); downscaled far-infrared and optical radiation (magenta); total $\gamma$ rays (right black) with their decomposition into pion decay (red), inverse Compton (green), and bremsstrahlung (cyan) radiation. CR powers (dotted lines) for protons (red), Helium (blue), primary electrons (green), secondary electrons (cyan), and secondary positrons (magenta). From Strong et al. (2010).

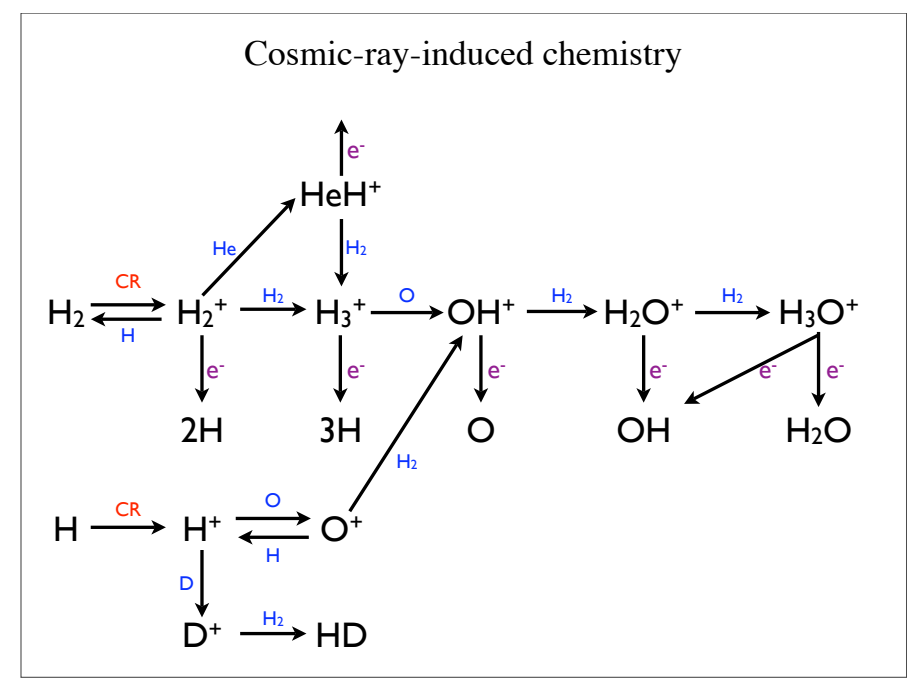

Figure 8: Flow chart of chemical reactions that are initiated by CR ionizations of $\mathrm{H}$ and $\mathrm{H}_{2}$. This simplified version illustrates how the abundances of the oxygencontaining ions and $\mathrm{H}_{3}^{+}$are related to the ionization rate and to the fractional abundances of $\mathrm{H}_{2}$ and free electrons. 


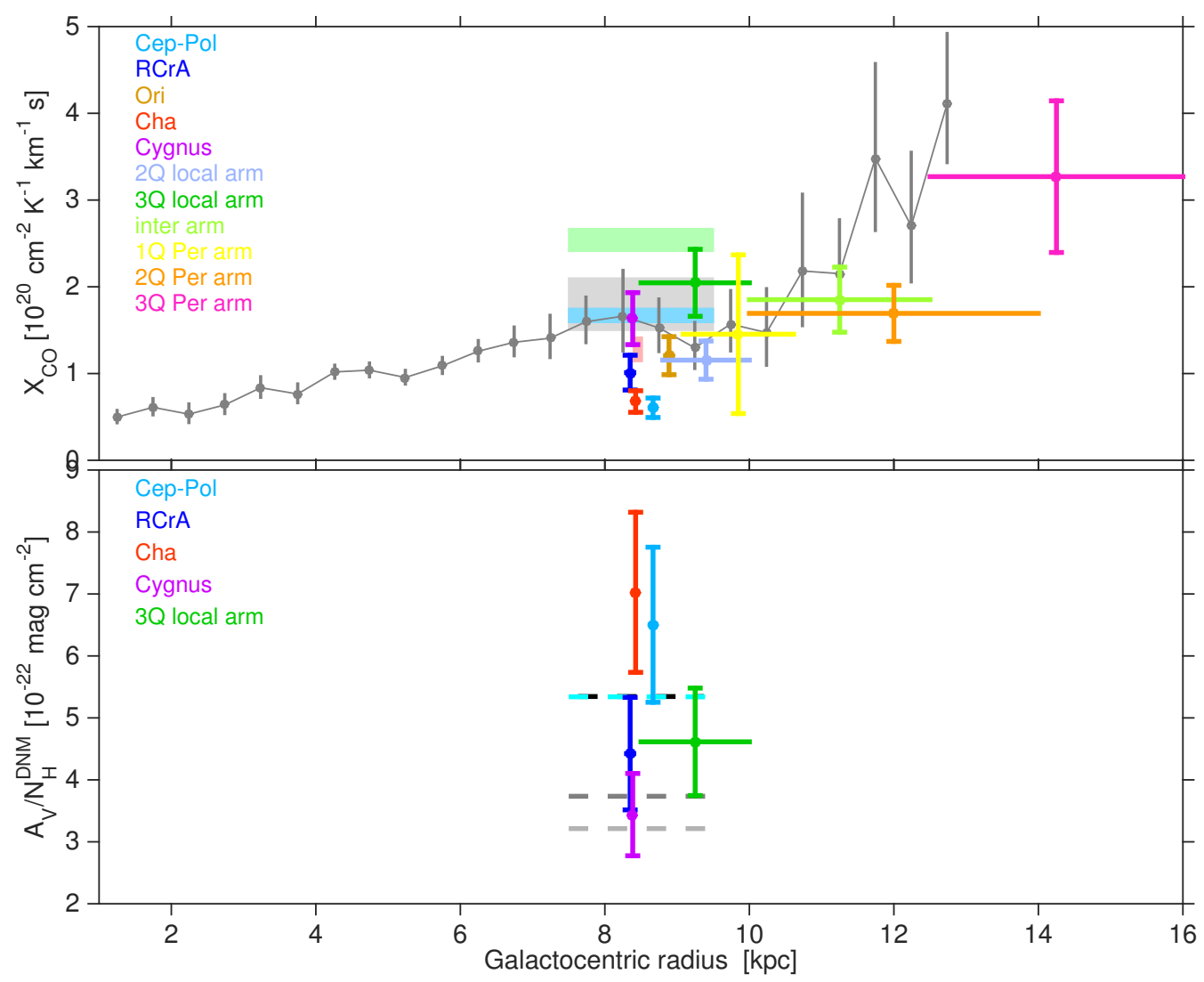

Figure 9: Upper panel: radial distribution across the Milky Way of the $X_{\mathrm{CO}}$ ratios measured in $\gamma$ rays (same data, colours, and errors as in Fig. 4), and from the radiative transfer of ${ }^{12} \mathrm{CO}$ and ${ }^{13} \mathrm{CO}$ lines for a given gradient in $[\mathrm{C}] /[\mathrm{H}]$ abundance (grey profile, from Pineda et al. 2013). The $\gamma$-ray measure assumes no metallicity or CR-density gradient. Examples of dust-derived ratios are given as rectangles. Estimates based on dust emissivity spectra and optical depth exceed the $\gamma$-ray values found in the same region (e.g., pink rectangle in the Chamaeleon from Planck et al. (2014), or green rectangle at $|b|>10^{\circ}$ from Planck Collaboration et al. (2011b)). Estimates from dust absorption (blue, Paradis et al. 2012) or 100- $\mu$ m flux (grey, Dame, Hartmann \& Thaddeus 2001) are consistent with the other local data. Lower panel: local $\gamma$-ray measurements of the $A_{V} / N_{\mathrm{H}}$ ratios in the DNM phase, compared with dust estimates in the HI (dashed black, grey, and lightgrey lines respectively for Bohlin, Savage \& Drake 1978; Liszt 2014; Planck Collaboration et al. 2014b), and dust estimates in the local DNM (dashed cyan, Planck Collaboration et al. 2011b). 


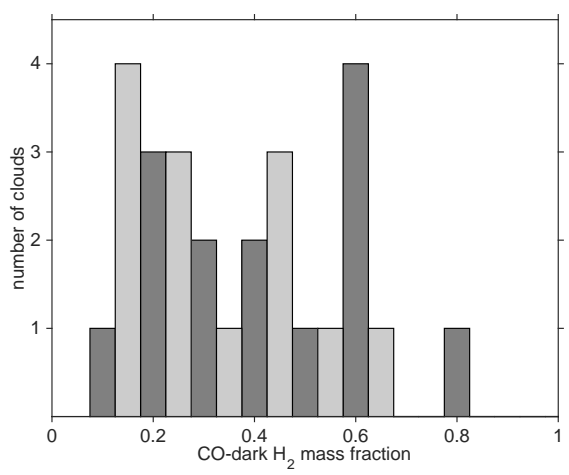

Figure 10: Mass fractions of the $\mathrm{H}_{2}$ gas that is CO-dark in nearby clouds, as traced with CRs and dust (Abdo et al. 2010g; Ackermann et al. 2012d,f; Grenier, Casandjian \& Terrier 2005; Planck et al. 2014). The fractions are given for a half (light) or fully (dark) molecular DNM composition.

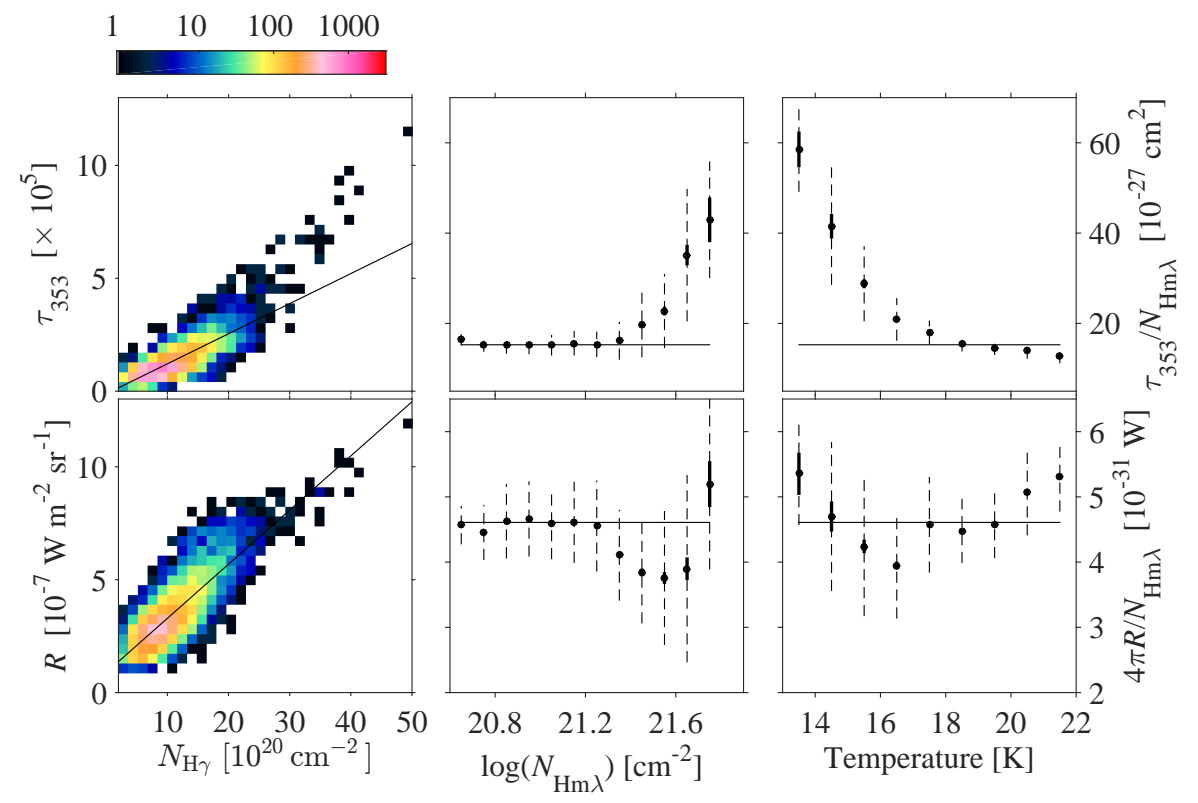

Figure 11: Evolution of the dust emission properties in the Chamaeleon clouds, adapted from Planck et al. (2014). The left column shows 2D histograms of the correlations between the total gas column densities $N_{\mathrm{H} \gamma}$ measured in $\gamma$ rays, and the dust optical depth at $353 \mathrm{GHz}, \tau_{353}$, or the dust radiance, $R$, deduced from the 353-3000 $\mathrm{GHz}$ spectral energy distributions of the thermal emission of the grains. The other columns show the changes in dust emission opacity, $\tau_{353} / N_{\mathrm{H}}$, and specific power, $4 \pi R / N_{\mathrm{H}}$, in intervals of $N_{\mathrm{Hm} \lambda}$ column density (middle) and dust colour temperature (right). The $N_{\mathrm{Hm} \lambda}$ values come from the higher-resolution multi-wavelength data, gauged in column densities with the $\gamma$ rays (details in the publication). The solid and dashed error bars respectively give the error on the means and the standard deviations in each sample. The thin lines mark the average values found in the $(0.4-2) \times 10^{21} \mathrm{~cm}^{2}$ range. 


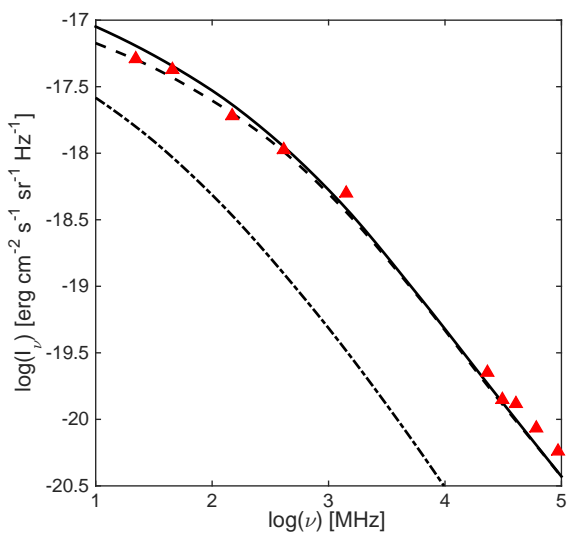

Figure 12: Synchrotron spectra at high latitudes, $10^{\circ} \leq|b| \leq 45^{\circ}$, from primary electrons (dashed), secondary electrons and positrons (dot-dashed), and total leptons (solid). Data points (triangles) are from various radio surveys below 10 GHz, and WMAP from $23 \mathrm{GHz}$. From Orlando \& Strong (2013). 


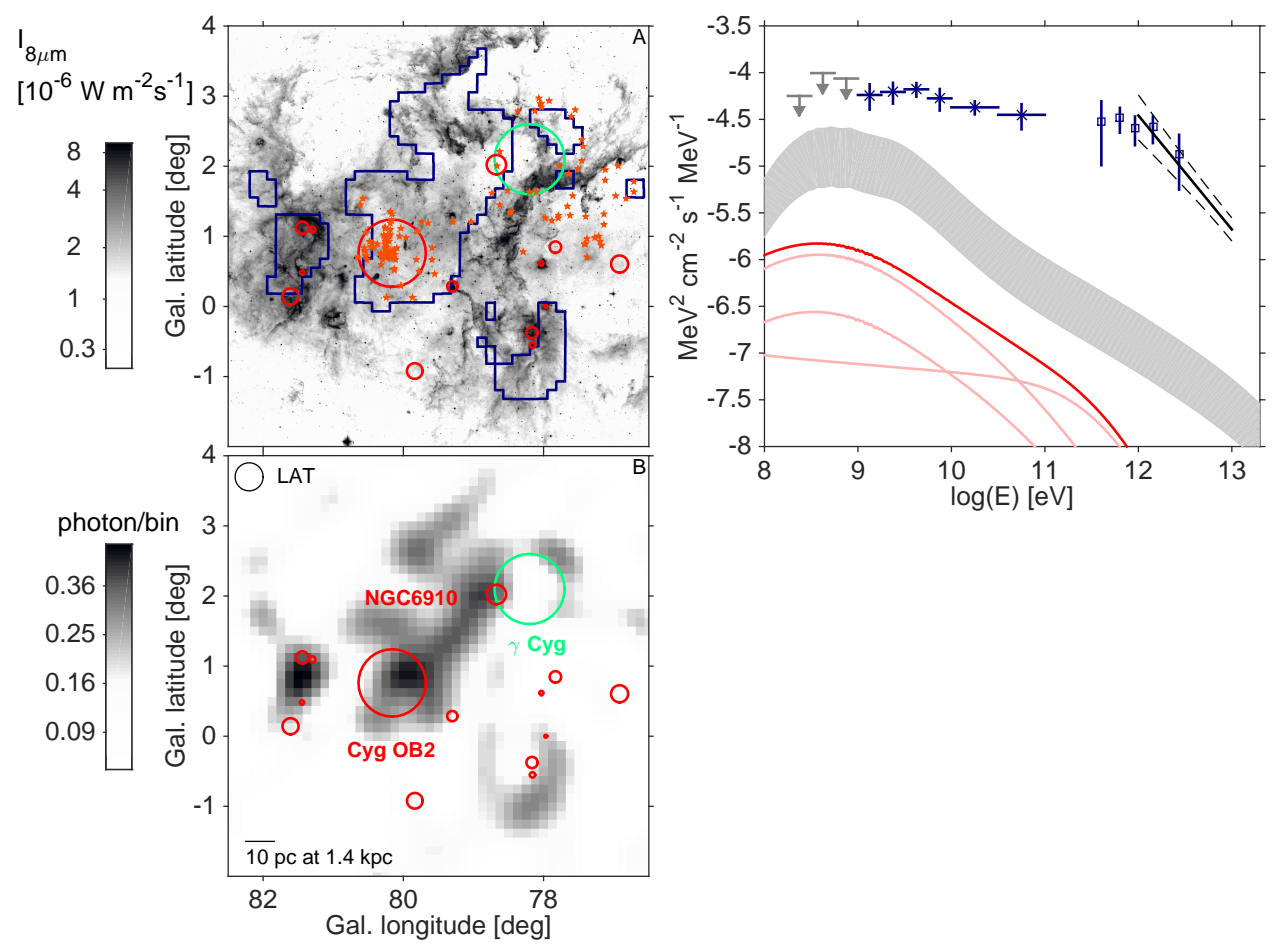

Figure 13: The Cygnus $\mathrm{X}$ region: maps (A) of the $8-\mu \mathrm{m}$ emission from photodominated regions (MSX data) and (B) of the photon excess detected in the 10-100 GeV band by Fermi LAT (Ackermann et al. 2011a). The $\gamma$-ray excess reveals young cosmic rays diffusing in the ionized cavities carved by numerous OB stars $(*)$ and $\mathrm{OB}$ associations (red circles). The $0.2 \gamma /$ bin contour of the $\gamma$-ray excess is overlaid in blue in plot A. The green circle marks the $\gamma$ Cygni supernova remnant. The $\gamma$-ray emission from its disc has been subtracted. Right: spectral energy distribution of the emission detected by the LAT (crosses, Ackermann et al. 2011a), MILAGRO (dashed power law, Abdo et al. 2012), and ARGO-YBG (squares, Bartoli et al. 2014). The curves show the expectations from the old Galactic CR population spreading the ionized gas (grey band), or up-scattering the stellar and interstellar light (red curve) with individual contributions from the Cyg OB2 cluster (upper pink), the NGC 6910 cluster (middle pink), and the average interstellar radiation field in the region (lower pink). 